\title{
Chemical Abundances of Main-sequence, Turnoff, Subgiant, and Red Giant Stars from APOGEE Spectra. II. Atomic Diffusion in M67 Stars
}

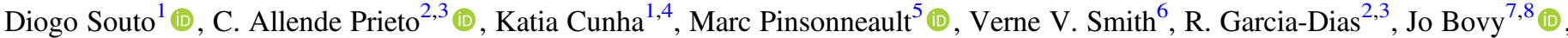 \\ D. A. García-Hernández ${ }^{2,3}$, Jon Holtzman ${ }^{9}$ (D), J. A. Johnson ${ }^{5}$ (D), Henrik Jönsson ${ }^{10}$ (D), Steve R. Majewski ${ }^{11}$ (D), \\ Matthew Shetrone ${ }^{12}$ (D), Jennifer Sobeck ${ }^{11}$, Olga Zamora ${ }^{2,3}$, Kaike Pan ${ }^{13}$ (D), and Christian Nitschelm ${ }^{14}$ \\ ${ }^{1}$ Observatório Nacional, Rua General José Cristino, 77, 20921-400 São Cristóvão, Rio de Janeiro, RJ, Brazil; souto@on.br, diogodusouto@gmail.com \\ ${ }^{2}$ Instituto de Astrofísica de Canarias, E-38205 La Laguna, Tenerife, Spain \\ ${ }^{3}$ Departamento de Astrofísica, Universidad de La Laguna, E-38206 La Laguna, Tenerife, Spain \\ ${ }^{4}$ Steward Observatory, University of Arizona, 933 North Cherry Avenue, Tucson, AZ 85721-0065, USA \\ ${ }^{5}$ Department of Astronomy, The Ohio State University, Columbus, OH 43210, USA \\ ${ }^{6}$ National Optical Astronomy Observatory, 950 North Cherry Avenue, Tucson, AZ 85719, USA \\ ${ }^{7}$ Department of Astronomy and Astrophysics, University of Toronto, 50 St. George Street, Toronto, ON, M5S 3H4, Canada \\ ${ }^{8}$ Dunlap Institute for Astronomy and Astrophysics, University of Toronto, ON M5S 3H4, Canada \\ ${ }^{9}$ New Mexico State University, Las Cruces, NM 88003, USA \\ ${ }^{10}$ Lund Observatory, Department of Astronomy and Theoretical Physics, Lund University, Box 43, SE-221 00 Lund, Sweden \\ ${ }^{11}$ Department of Astronomy, University of Virginia, Charlottesville, VA 22904-4325, USA \\ ${ }^{12}$ University of Texas at Austin, McDonald Observatory, USA \\ ${ }^{13}$ Apache Point Observatory and New Mexico State University, P.O. Box 59, Sunspot, NM, 88349-0059, USA \\ ${ }^{14}$ Centro de Astronomía (CITEVA), Universidad de Antofagasta, Avenida Angamos 601, Antofagasta 1270300, Chile \\ Received 2018 December 23; revised 2019 February 25; accepted 2019 February 26; published 2019 March 27
}

\begin{abstract}
Chemical abundances for 15 elements (C, N, O, Na, Mg, Al, Si, K, Ca, Ti, V, Cr, Mn, Fe, and Ni) are presented for 83 stellar members of the 4 Gyr old solar-metallicity open cluster M67. The sample contains stars spanning a wide range of evolutionary phases, from $\mathrm{G}$ dwarfs to red clump stars. The abundances were derived from near-IR $(\lambda 1.5-1.7 \mu \mathrm{m})$ high-resolution spectra $(R=22,500)$ from the SDSS-IV/Apache Point Observatory Galactic Evolution Experiment (APOGEE) survey. A 1D local thermodynamic equilibrium abundance analysis was carried out using the APOGEE synthetic spectral libraries, via $\chi^{2}$ minimization of the synthetic and observed spectra with the qASPCAP code. We found significant abundance differences $(\sim 0.05-0.30$ dex $)$ between the M67 member stars as a function of the stellar mass (or position on the Hertzsprung-Russell diagram), where the abundance patterns exhibit a general depletion (in $[\mathrm{X} / \mathrm{H}]$ ) in stars at the main-sequence turnoff. The amount of the depletion is different for different elements. We find that atomic diffusion models provide, in general, good agreement with the abundance trends for most chemical species, supporting recent studies indicating that measurable atomic diffusion operates in M67 stars.
\end{abstract}

Key words: diffusion - infrared: stars - open clusters and associations: general - stars: abundances

\section{Introduction}

M67 (Messier 67; NGC 2682) is a well-studied open cluster, with an age and metallicity $(4 \mathrm{Gyr}$ and $[\mathrm{Fe} / \mathrm{H}]=0.0$, respectively) similar to those of the Sun. A number of studies have determined the distance to the cluster (Yadav et al. 2008), its age (Yadav et al. 2008, Sarajedini et al. 2009), photometric colors and reddening (Taylor 2007, Sarajedini et al. 2009), as well as metallicity and individual chemical abundances (Cohen 1980, Foy \& Proust 1981; Tautvaišiene et al. 2000; Pancino et al. 2010; Jacobson et al. 2011; Önehag et al. 2014; Liu et al. 2016; Bertelli Motta et al. 2018; Gao et al. 2018, and Souto et al. 2018). M67 is a "benchmark" Galactic open cluster and an excellent laboratory in which to study poorly understood processes in stellar astrophysics, such as abundance variations in open clusters.

The chemical composition of a star is inherited from the interstellar matter from which it forms; however, this composition changes over time due to internal stellar processes, such as gravitational settling or atomic diffusion. The approximation employed in the determination of abundances can also induce systematic errors in the inferred abundances, creating an apparent lack of homogeneity. Examples of such simplifications are the assumptions of hydrostatic equilibrium or local thermodynamic equilibrium (LTE).
Stellar clusters are useful astrophysical environments to study elemental abundance variations because of the reasonable assumption that stars in a cluster were born from the same molecular cloud at the same time. Several authors have studied the initial chemical homogeneity of open and globular clusters (De Silva et al. 2006, 2007, Reddy et al. 2012; Bovy 2016) and have, so far, not found any evidence of inhomogeneities in the initial stellar populations of open and globular clusters.

One well-known process that has been extensively observed in clusters is that as stars evolve into red giants, their surface carbon and nitrogen abundances are altered by the convectively driven first dredge-up of material from the stellar interior that has been exposed to $\mathrm{H}$ burning via the $\mathrm{CN}$ cycle (Bressan et al. 2012; Lagarde et al. 2012; Choi et al. 2016). This process does not, however, explain the lack of uniformity in the elemental abundances of main-sequence and turnoff stars found in metalpoor globular clusters (Korn et al. 2007, Lind et al. 2008; Nordlander et al. 2012). These variations are instead explained by atomic diffusion, a fundamental process predicted by theory (Michaud et al. 2015, references therein) and operating in all stars, which is often ignored in stellar evolution models and abundance studies. Atomic diffusion represents the physical process that involves the transport of material in the stellar atmosphere that is described by a diffusion equation, for 
example, gravitational settling. Atomic diffusion has a physical basis, with diffusion coefficients predicted by theory; see Chapman (1917a, 1917b), Aller \& Chapman (1960), Michaud et al. (1976), Vauclair et al. (1978), Michaud (1980), Vauclair \& Vauclair (1982), and Michaud et al. (2004).

Diffusion in stars having a solar age and metallicity, as is the case for members of M67, has been theoretically investigated by Michaud et al. (2004), who analyzed 28 elements, finding that $\mathrm{He}, \mathrm{Li}, \mathrm{Be}, \mathrm{B}, \mathrm{Mg}, \mathrm{P}, \mathrm{Ti}, \mathrm{Fe}$, and $\mathrm{Ni}$ were those most affected by this mechanism. One of their conclusions was that atomic diffusion models can have a significant impact on the stellar ages derived from isochrones. More recently, theoretical calculations by Dotter et al. (2017) concluded that atomic diffusion also plays an important role in stars with a solar age and metallicity (not only metal-poor stars), and they found that the photospheric iron abundance in turnoff stars can be depleted by $\sim 0.12$ dex compared to their initial surface abundance as a consequence of atomic diffusion processes. Dotter et al. (2017) noted that ignoring diffusion in models would cause an additional uncertainty of about $10 \%$ in the stellar ages derived from isochrones.

Evidence for the occurrence of diffusion in M67 stars has been found previously by Önehag et al. (2014), who studied a sample of 14 stars belonging to M67, including main-sequence stars (six), turnoff (three), and the early subgiant branch (five), using high-resolution optical spectra from FLAMES/UVES on the Very Large Telescope (VLT). Önehag et al. (2014) found abundance differences among the groups of 0.05-0.10 dex for $\mathrm{Al}, \mathrm{Ca}, \mathrm{Cr}, \mathrm{Mn}$, and $\mathrm{Fe}$, with turnoff stars having lower abundances than subgiants. Blanco-Cuaresma et al. (2015) compiled a sample of 42 stars in M67 (28 main sequence and 14 red giants) using spectra from the ESPaDOnS twin spectropolarimeter atop Pic du Midi (NARVAL), High Accuracy Radial velocity Planet Searcher (HARPS), and Ultraviolet and Visual Echelle Spectrograph (UVES). The authors observed that the abundances of $\mathrm{Na}, \mathrm{Mg}$, and $\mathrm{Si}$ show variations of up to $0.10-0.20$ dex between dwarf and giant stars in the cluster.

Souto et al. (2018, Paper I) studied a small sample of eight M67 stellar members spanning a range of evolutionary phases, including G dwarfs (two), G-turnoff stars (two), G subgiants (two), and red clump $\mathrm{K}$ giants (two) using high-resolution spectra from the Apache Point Observatory Galactic Evolution Experiment (APOGEE; Majewski et al. 2017). They found abundance variations in 14 elements across the HertzsprungRussell (H-R) diagram, confirming that most chemical species display changes in the range of 0.05-0.20 dex (Fe, Na, Mg, Al, $\mathrm{Si}, \mathrm{Ca}$, and $\mathrm{Mn}$ ), with the lower abundances observed in turnoff stars, with $M \sim 1.2 M_{\odot}$. Souto et al. (2018) also showed that the abundance variations found in M67 stars compare very well with theoretical models of atomic diffusion for stars having solar age and metallicity. Also using APOGEE spectra, Bertran de Lis et al. (2016) found significantly more dispersion in $[\mathrm{O} / \mathrm{Fe}]$ for M67 stars than for other clusters with similar metallicity but younger ages, such as NGC 6819 or NGC 2158. Bovy (2016) and Price-Jones \& Bovy (2018) found strong constraints on the chemical homogeneity in M67 red giant stars from APOGEE. The authors showed that M67 red giants are homogeneous based only on their stellar spectra, without the need of modeling the stellar atmosphere. The uniformity within the red giant stars may indicate that changes in the stellar abundances across different evolutionary phases in the H-R diagram for M67 might be related to physical processes operating within these stars.

The works of Bertelli Motta et al. (2018) and Gao et al. (2018) have confirmed, using independent data, that atomic diffusion operates in M67 stars. Both works used highresolution optical spectra. Bertelli Motta et al. (2018) used UVES/FLAMES ( $R \sim 20,000-32,000)$ observations from the Gaia/ESO survey (Gilmore et al. 2012, Randich et al. 2013), reporting abundances of 11 elements in 15 stars from the main sequence, turnoff, and red giant branch (RGB). Bertelli Motta et al. (2018), using APOGEE data, find abundance variations of up to $0.20-0.30$ dex for elements like $\mathrm{Al}, \mathrm{Mn}$, and $\mathrm{Ni}$, where non-LTE effects are unlikely to explain the observed trends. Gao et al. (2018) use spectra from the GALAH survey (De Silva et al. 2015), with a resolving power of $R \sim 28,000$, to report abundances for seven elements in 66 stars from the turnoff, subgiant, red giant, and red clump phases. Gao et al. (2018) conclude that deviations from non-LTE can explain some of the observed abundance trends as a function of the evolutionary stage, in particular for oxygen and sodium. However, for $\mathrm{Al}$ and $\mathrm{Si}$, non-LTE does not explain the remaining trend, which the authors argue might be a consequence of diffusion processes in M67.

This work provides a complementary verification of the atomic diffusion mechanisms acting in M67 stars as reported by Souto et al. (2018). We use APOGEE results obtained with the qASPCAP ${ }^{15}$ pipeline using a much larger stellar sample; qASPCAP is a simple IDL script that substitutes the entire ASPCAP (APOGEE Stellar Parameters and Chemical Abundances Pipeline, García Pérez et al. 2016) for boutique work.

APOGEE targeted M67 as one of its calibration clusters, observing about 100 stellar members from the lower main sequence, the turnoff, the subgiant branch, and the RGB. The M67 APOGEE sample is well suited both to probe the limits on chemical homogeneity in the cluster members and to search for signatures of atomic diffusion in the chemical abundances of a number of elements. APOGEE spectra are used here to derive detailed chemical abundances of 15 elements: $\mathrm{C}, \mathrm{N}, \mathrm{O}, \mathrm{Na}, \mathrm{Mg}$, $\mathrm{Al}, \mathrm{Si}, \mathrm{K}, \mathrm{Ca}, \mathrm{Ti}, \mathrm{V}, \mathrm{Cr}, \mathrm{Mn}, \mathrm{Fe}$, and $\mathrm{Ni}$.

The paper is structured as follows: in Section 2 we describe the adopted sample, in Section 3 we report on the atmospheric parameters and the methodology employed to derive the individual abundances, in Section 4 we analyze the abundance trends, and in Section 5 we suggest possible explanations for them. In Section 6 we discuss the obtained results, summarizing in Section 7.

\section{APOGEE Data on M67}

The APOGEE spectrographs are cryogenic multifiber near-infrared instruments covering the $H$ band between $\lambda 1.51 \mu \mathrm{m}$ and $\lambda 1.69 \mu \mathrm{m}$, obtaining high-resolution ( $R=\lambda / \Delta \lambda \sim 22,500$ ) spectra for 300 objects at a time (Gunn et al. 2006; Wilson et al. 2010). The spectrographs are currently mounted in both hemispheres on $2.5 \mathrm{~m}$ telescopes at APO (Apache Point Observatory, New Mexico, USA) and at LCO (Las Campanas Observatory, La Serena, Chile). The M67 stellar spectra analyzed in this work were all obtained at APO and reduced with the APOGEE pipeline, described in Nidever et al. (2015).

\footnotetext{
15 github.com/callendeprieto/
} 

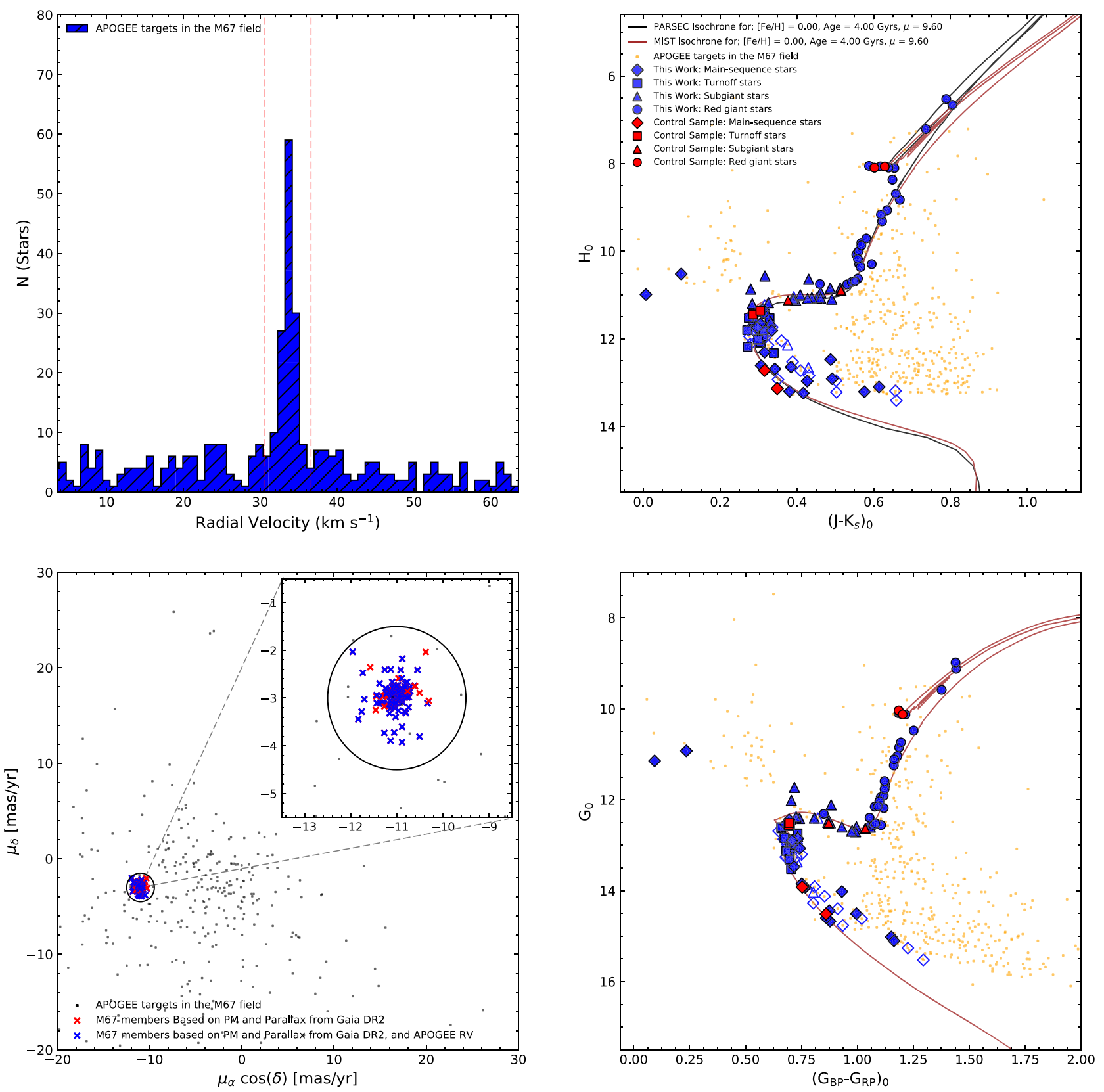

Figure 1. Top left panel: radial velocity distribution obtained from ASPCAP for all of the targets observed in the APOGEE M67 field. Bottom left panel: vector-point diagram with the adopted stellar proper motions. Top right panel: $\left(J-K_{s}\right)_{0}$ vs. $H_{0}$ diagram showing the sample of this work. We represent the main-sequence stars as blue diamonds, blue squares are the turnoff stars, the blue triangles are the subgiants, and the blue circles represent the red giant stars. We also include the stellar sample of Souto et al. (2018) using the same symbol notation for the stellar classes, but colored as red. The 2MASS color-magnitude diagram of the APOGEE targets in the M67 field is shown as orange dots. Two isochrones for an age of $4 \mathrm{Gyr},(m-M)_{0}=9.60$, and $[\mathrm{Fe} / \mathrm{H}]=0.00$ from PARSEC (black line) and the MESA Isochrones and Stellar Tracks (MIST; brown line) are also shown. We left as open symbols the stars with $\mathrm{S} / \mathrm{N}<100$. Bottom right panel: same as top right panel, expect the color-magnitude diagram using Gaia DR2 data for $\left(G_{\mathrm{BP}}-G_{\mathrm{RP}}\right)_{0}$ vs. $G_{0}$.

APOGEE is part of the SDSS-III and SDSS-IV projects (Eisenstein et al. 2011, Blanton et al. 2017), and M67 is one of the calibration clusters for the ASPCAP pipeline (Mészáros et al. 2013; Zasowski et al. 2013; Holtzman et al. 2015; García Pérez et al. 2016). APOGEE has observed a dedicated field in the direction of M67 (location ID 4162), obtaining spectra for 563 targets. The stars had multiple visits, generally more than three, to reach the required signal-to-noise ratio $(\mathrm{S} / \mathrm{N})$ of the combined spectra (higher than $\sim 100$ per half a resolution element); this was achieved for stars brighter than $H \leqslant 11$.

To verify membership of the observed stars in the M67 APOGEE field, we adopt two approaches, one using membership studies from the literature and another using distances and proper motions from Gaia DR2 (Gaia Collaboration et al. 2018). We initially used the radial velocities (RV) measured by the APOGEE pipeline available in the 14th SDSS data release (DR14, Abolfathi et al. 2018), following the proper motion and RV membership criteria of Yadav et al. (2008) and Geller et al. (2015) as guidelines. Yadav et al. (2008) determined proper motions for 2462 stars using the Wide-Field-Imager from the MPG/ESO $2.2 \mathrm{~m}$ telescope at La Silla, Chile, with a field of view of $34 \times$ $33 \mathrm{arcmin}^{2}$. The authors reported 434 stars having membership probabilities $\geqslant 90 \%$. Using the same data, Bellini et al. (2010) derived the cluster average proper motion to be $\mu_{\alpha}$ 


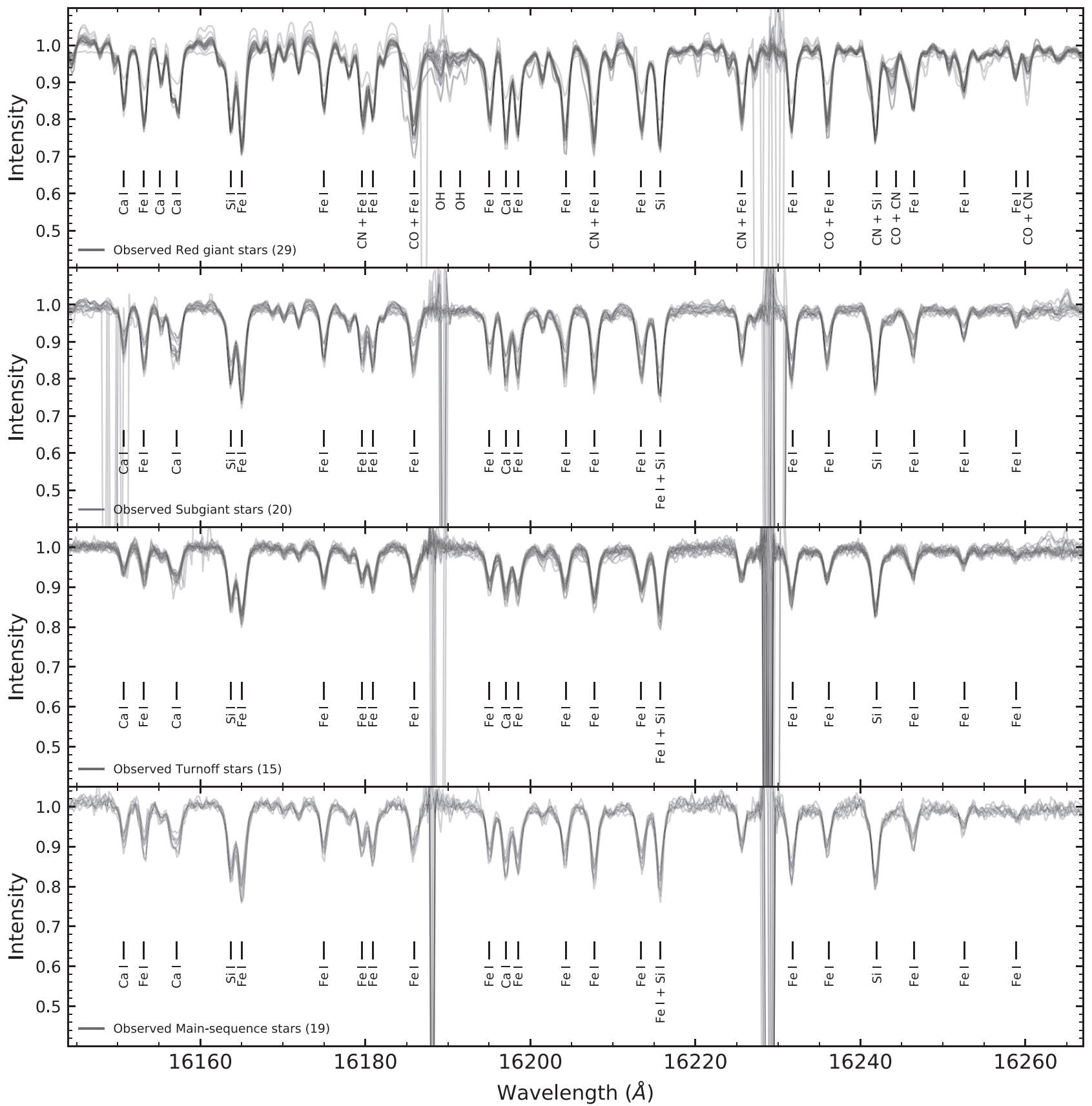

Figure 2. Portion of the APOGEE observed spectra for the stellar sample. From top to bottom, we shown the spectra of red giant, subgiant, turnoff, and main-sequence stars.

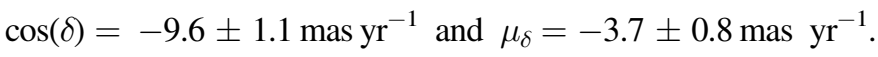
The radial velocity survey by Geller et al. (2015) used spectra obtained from various sources, including a total of 1278 stars in the vicinity of M67. Geller et al. (2015) reported 590 stars having membership probabilities $\geqslant 90 \%$, where the mean radial velocity of the sample is $33.64 \mathrm{~km} \mathrm{~s}^{-1}$, with high internal precision $\left(0.03 \mathrm{~km} \mathrm{~s}^{-1}\right)$.

Based on this information, an initial membership cut was performed, selecting from the targets observed in the M67 APOGEE field (563 stars), those within the radial velocity range $30.64-36.64 \mathrm{~km} \mathrm{~s}^{-1}$. Figure 1 (top left panel) shows a histogram of the RV distribution of all the stars in the field. The peak of the RV distribution compares well with the mean radial velocity for the cluster reported by Geller et al. (2015), with
140 stars falling within the RV limit (red dashed lines). We then performed a cross-match between the stars within the limit in radial velocity and those stars reported by Yadav et al. (2008) and Geller et al. (2015) having membership probabilities $\geqslant 90 \%$. A total of 119 stars satisfied these criteria.

We then adopted Gaia DR2 (Gaia Collaboration et al. 2018) proper motions with distances from Bailer-Jones et al. (2018) to refine the sample. From those 140 stars within the RV limits, we find 109 within the ranges in distance and proper motion for M67. We accepted stars with distances in the range $796.2-992.0 \mathrm{pc}$, which corresponds to a distance modulus of 9.56-9.88, as reported in the literature for the cluster (Yadav et al. 2008, Yakut et al. 2009). We then adopted the mean proper motions observed for the stars within the adopted 
Table 1

Stellar Properties

\begin{tabular}{|c|c|c|c|c|c|c|c|c|c|c|c|c|c|c|c|c|c|}
\hline 2Mass ID & $\begin{array}{c}\mathrm{RV} \\
\left(\mathrm{km} \mathrm{s}^{-1}\right)\end{array}$ & $\begin{array}{c}\sigma(\mathrm{RV}) \\
\sigma(\mathrm{km} \\
\left.\mathrm{s}^{-1}\right)\end{array}$ & $\begin{array}{l}\text { PM (R.A.) } \\
\left(\mu_{\alpha} \cos (\delta)\right)\end{array}$ & $\begin{array}{c}\text { PM } \\
\text { (R.A.) } \\
\\
\quad \sigma\end{array}$ & $\begin{array}{c}\text { PM } \\
\text { (Decl.) } \\
\left(\mu_{\delta}\right)\end{array}$ & $\begin{array}{c}\text { PM } \\
\text { (Decl.) } \\
\\
\sigma\end{array}$ & $\begin{array}{c}\text { Dist BJ18 } \\
\text { parsec }\end{array}$ & $\begin{array}{l}\text { Dist }(\sigma) \\
\text { parsec }\end{array}$ & $\mathrm{S} / \mathrm{N}$ & $\begin{array}{l}\text { Prob } \\
\text { G15 }\end{array}$ & Y08 & $\begin{array}{l}\text { Prob } \\
\text { Z93 }\end{array}$ & $\begin{array}{l}\text { Prob } \\
\text { G89 }\end{array}$ & $\begin{array}{l}\text { Prob } \\
\text { S77 }\end{array}$ & $J$ & $H$ & $K_{s}$ \\
\hline Red Giants & & & & & & & & & & & & & & & & & \\
\hline $\begin{array}{r}2 \mathrm{M} 08492491 \\
+1144057\end{array}$ & 35.09 & 1.51 & -11.06 & 0.07 & -2.87 & 0.05 & 829.45 & 28.15 & 460 & 98 & 99 & 99 & 99 & 2 & 10.296 & 9.831 & 9.708 \\
\hline $\begin{array}{r}2 \mathrm{M} 08503613 \\
+1143180\end{array}$ & 34.29 & 0.11 & -11.06 & 0.07 & -2.74 & 0.06 & 873.89 & 24.21 & 138 & 72 & 100 & 93 & 97 & 94 & 11.131 & 10.644 & 10.552 \\
\hline $\begin{array}{r}2 \mathrm{M} 08504964 \\
+1135089\end{array}$ & 34.92 & 0.07 & -10.96 & 0.08 & -2.96 & 0.06 & 832.42 & 28.06 & 344 & 98 & 99 & 94 & 99 & 95 & 9.410 & 8.848 & 8.722 \\
\hline $\begin{array}{r}2 \mathrm{M} 08511269 \\
+1152423\end{array}$ & 34.34 & 0.07 & -10.95 & 0.06 & -2.98 & 0.04 & 818.41 & 12.56 & 1445 & 98 & 99 & 99 & 96 & 95 & 8.650 & 8.122 & 7.976 \\
\hline $\begin{array}{r}2 \mathrm{M} 08511704 \\
+1150464\end{array}$ & 33.58 & 0.06 & -11.16 & 0.07 & -3.32 & 0.05 & 829.68 & 28.19 & 371 & 98 & 99 & 77 & 97 & 95 & 9.284 & 8.712 & 8.606 \\
\hline $\begin{array}{r}2 \mathrm{M} 08511897 \\
+1158110\end{array}$ & 34.01 & 0.10 & -11.08 & 0.06 & -3.09 & 0.04 & 847.08 & 23.29 & 384 & 98 & 100 & 94 & 98 & 51 & 10.587 & 10.095 & 10.012 \\
\hline $\begin{array}{r}2 \mathrm{M} 08512156 \\
+1146061\end{array}$ & 34.87 & 0.06 & -11.10 & 0.08 & -2.66 & 0.05 & 834.37 & 31.65 & 314 & 97 & 98 & 91 & 99 & 95 & 9.602 & 9.085 & 8.947 \\
\hline $\begin{array}{r}2 \mathrm{M} 08512618 \\
+1153520\end{array}$ & 34.16 & 0.04 & -11.00 & 0.07 & -2.88 & 0.05 & 842.43 & 14.79 & 982 & 97 & 97 & 77 & 99 & 95 & 8.619 & 8.113 & 7.960 \\
\hline $\begin{array}{r}2 \mathrm{M} 08512898 \\
+1150330\end{array}$ & 33.46 & 0.04 & -11.14 & 0.08 & -3.22 & 0.05 & 812.29 & 18.46 & 481 & 98 & 100 & 94 & 98 & 95 & 8.566 & 8.072 & 7.958 \\
\hline $\begin{array}{r}2 \mathrm{M} 08512990 \\
+1147168\end{array}$ & 36.28 & 0.01 & -11.27 & 0.09 & -3.73 & 0.05 & 795.23 & 34.60 & 884 & 98 & 99 & 0 & 96 & 96 & 7.314 & 6.681 & 6.489 \\
\hline $\begin{array}{r}2 \mathrm{M} 08513577 \\
+1153347\end{array}$ & 34.05 & 0.11 & -11.06 & 0.06 & -2.93 & 0.04 & 801.49 & 20.07 & 205 & 98 & 93 & 99 & 72 & 95 & 10.522 & 10.023 & 9.941 \\
\hline $\begin{array}{r}2 \mathrm{M} 08513938 \\
+1151456\end{array}$ & 33.98 & 0.11 & -11.10 & 0.07 & -3.12 & 0.04 & 834.61 & 26.61 & 469 & 98 & 100 & 95 & 99 & 93 & 10.383 & 9.889 & 9.795 \\
\hline $\begin{array}{r}2 \mathrm{M} 08514234 \\
+1150076\end{array}$ & 34.27 & 0.05 & -11.02 & 0.07 & -2.80 & 0.05 & 805.95 & 23.64 & 271 & 98 & 0 & 99 & 99 & 96 & 9.829 & 9.339 & 9.187 \\
\hline $\begin{array}{r}2 \mathrm{M} 08514388 \\
+1156425\end{array}$ & 32.94 & 0.05 & -11.18 & 0.11 & -3.16 & 0.07 & 844.60 & 22.86 & 505 & 95 & 100 & 99 & 98 & 91 & 8.618 & 8.114 & 7.996 \\
\hline $\begin{array}{r}2 \mathrm{M} 08514507 \\
+1147459\end{array}$ & 32.97 & 0.04 & -11.05 & 0.07 & -3.03 & 0.04 & 839.67 & 26.03 & 281 & 97 & 99 & 2 & 92 & 92 & 9.684 & 9.183 & 9.045 \\
\hline $\begin{array}{r}2 \mathrm{M} 08514883 \\
+1156511\end{array}$ & 34.35 & 0.05 & -10.96 & 0.08 & -3.26 & 0.05 & 858.95 & 35.02 & 135 & 97 & 99 & 99 & 99 & 94 & 11.256 & 10.779 & 10.705 \\
\hline $\begin{array}{r}2 \mathrm{M} 08515611 \\
+1150147\end{array}$ & 34.68 & 0.04 & -11.13 & 0.08 & -3.89 & 0.05 & 843.67 & 28.77 & 133 & 98 & 99 & 99 & 98 & 95 & 11.197 & 10.726 & 10.634 \\
\hline $\begin{array}{r}2 \mathrm{M} 08515952 \\
+1155049\end{array}$ & 34.39 & 0.05 & -11.00 & 0.09 & -3.10 & 0.06 & 868.56 & 21.45 & 543 & 98 & 99 & 88 & 91 & 90 & 8.597 & 8.084 & 7.959 \\
\hline $\begin{array}{r}2 \mathrm{M} 08521097 \\
+1131491\end{array}$ & 33.82 & 0.03 & -11.06 & 0.06 & -2.76 & 0.04 & 822.72 & 23.87 & 672 & 98 & 100 & 92 & 98 & 96 & 8.921 & 8.388 & 8.252 \\
\hline $\begin{array}{r}2 \mathrm{M} 08521656 \\
+1119380\end{array}$ & 33.82 & 0.03 & -11.05 & 0.07 & -2.88 & 0.05 & 808.61 & 30.73 & 1073 & 97 & 100 & 71 & 38 & 94 & 7.875 & 7.233 & 7.119 \\
\hline $\begin{array}{r}2 \mathrm{M} 08521856 \\
+1144263\end{array}$ & 33.65 & 0.06 & -11.13 & 0.07 & -3.14 & 0.05 & 818.62 & 13.28 & 504 & 96 & 100 & 95 & 98 & 94 & 8.572 & 8.087 & 7.923 \\
\hline $\begin{array}{r}2 \mathrm{M} 08522636 \\
+1141277\end{array}$ & 33.41 & 0.10 & -10.77 & 0.08 & -2.99 & 0.05 & 784.76 & 28.62 & 196 & 97 & 99 & 99 & 99 & 0 & 10.845 & 10.314 & 10.263 \\
\hline
\end{tabular}


Table 1

(Continued)

\begin{tabular}{|c|c|c|c|c|c|c|c|c|c|c|c|c|c|c|c|c|c|}
\hline 2Mass ID & $\begin{array}{c}\mathrm{RV} \\
\left(\mathrm{km} \mathrm{s}^{-1}\right)\end{array}$ & $\begin{array}{c}\sigma(\mathrm{RV}) \\
\sigma(\mathrm{km} \\
\left.\mathrm{s}^{-1}\right)\end{array}$ & $\begin{array}{l}\text { PM (R.A.) } \\
\left(\mu_{\alpha} \cos (\delta)\right)\end{array}$ & $\begin{array}{c}\text { PM } \\
\text { (R.A.) } \\
\\
\sigma\end{array}$ & $\begin{array}{c}\text { PM } \\
(\text { Decl. }) \\
\left(\mu_{\delta}\right)\end{array}$ & $\begin{array}{c}\text { PM } \\
\text { (Decl.) } \\
\\
\sigma\end{array}$ & $\begin{array}{c}\text { Dist BJ18 } \\
\text { parsec }\end{array}$ & $\begin{array}{l}\text { Dist }(\sigma) \\
\text { parsec }\end{array}$ & $\mathrm{S} / \mathrm{N}$ & $\begin{array}{l}\text { Prob } \\
\text { G15 }\end{array}$ & $\begin{array}{l}\text { Prob } \\
\text { Y08 }\end{array}$ & $\begin{array}{l}\text { Prob } \\
\text { Z93 }\end{array}$ & $\begin{array}{l}\text { Prob } \\
\text { G89 }\end{array}$ & $\begin{array}{l}\text { Prob } \\
\text { S77 }\end{array}$ & $J$ & $H$ & $K_{s}$ \\
\hline $\begin{array}{r}2 \mathrm{M} 08525625 \\
+1148539\end{array}$ & 32.84 & 0.07 & -11.02 & 0.08 & -3.10 & 0.05 & 870.52 & 33.96 & 195 & 97 & 99 & 99 & 99 & 77 & 10.839 & 10.315 & 10.224 \\
\hline $\begin{array}{r}2 \mathrm{M} 08534672 \\
+1123307\end{array}$ & 33.04 & 0.07 & -11.24 & 0.08 & -2.79 & 0.05 & 864.38 & 32.99 & 370 & nan & nan & nan & nan & nan & 10.225 & 9.730 & 9.624 \\
\hline $\begin{array}{r}2 \mathrm{M} 08493465 \\
+1151256\end{array}$ & 33.98 & 0.08 & -10.98 & 0.06 & -2.92 & 0.04 & 904.59 & nan & 1369 & 98 & 98 & 99 & 91 & 96 & 7.203 & 6.546 & 6.394 \\
\hline $\begin{array}{r}2 \mathrm{M} 08505816 \\
+1152223\end{array}$ & 34.03 & 0.11 & -11.13 & 0.08 & -2.86 & 0.05 & 884.25 & 34.01 & 287 & 98 & 99 & 99 & 96 & 91 & 11.197 & 10.707 & 10.626 \\
\hline $\begin{array}{r}2 \mathrm{M} 08510723 \\
+1153019\end{array}$ & 32.99 & 30.95 & -10.92 & 0.07 & -2.41 & 0.05 & 903.98 & 21.87 & 604 & 11 & 100 & 99 & 97 & 71 & 11.175 & 10.771 & 10.695 \\
\hline $\begin{array}{r}2 \mathrm{M} 08510839 \\
+1147121\end{array}$ & 33.52 & 0.18 & -10.91 & 0.08 & -2.93 & 0.06 & 888.63 & 28.60 & 171 & 98 & 98 & 99 & 99 & 93 & 10.691 & 10.195 & 10.112 \\
\hline $\begin{array}{r}2 \mathrm{M} 08522003 \\
+1127362\end{array}$ & 33.94 & 0.05 & -11.22 & 0.07 & -2.91 & 0.04 & 893.78 & 28.66 & 260 & 98 & 99 & 93 & 91 & 89 & 10.839 & 10.383 & 10.253 \\
\hline Subgiants & & & & & & & & & & & & & & & & & \\
\hline $\begin{array}{r}2 \mathrm{M} 08504994 \\
+1149127\end{array}$ & 33.83 & 0.10 & -10.83 & 0.07 & -3.27 & 0.05 & 809.79 & 28.26 & 110 & 98 & 100 & 94 & 97 & 93 & 11.372 & 10.960 & 10.890 \\
\hline $\begin{array}{r}2 \mathrm{M} 08510325 \\
+1145473\end{array}$ & 35.11 & 0.21 & -11.07 & 0.08 & -2.91 & 0.06 & 829.30 & 27.10 & 103 & 61 & 96 & 54 & 99 & 95 & 11.491 & 11.220 & 11.187 \\
\hline $\begin{array}{r}2 \mathrm{M} 08511564 \\
+1150561\end{array}$ & 34.01 & 0.03 & -10.73 & 0.07 & -2.78 & 0.05 & 785.07 & 21.58 & 209 & 86 & 100 & 99 & 98 & 94 & 11.485 & 11.094 & 11.013 \\
\hline $\begin{array}{r}2 \mathrm{M} 08511670 \\
+1145293\end{array}$ & 35.48 & 0.21 & -11.26 & 0.13 & -2.41 & 0.09 & 843.16 & 51.73 & 140 & nan & nan & nan & nan & nan & 11.021 & 10.662 & 10.570 \\
\hline $\begin{array}{r}2 \mathrm{M} 08512122 \\
+1145526\end{array}$ & 33.49 & 0.69 & -11.74 & 0.09 & -2.47 & 0.06 & 852.74 & 38.64 & 110 & 98 & 98 & 95 & 99 & 95 & 11.135 & 10.888 & 10.835 \\
\hline $\begin{array}{r}2 \mathrm{M} 08512879 \\
+1151599\end{array}$ & 33.59 & 0.13 & -10.91 & 0.07 & -3.04 & 0.05 & 840.04 & 26.19 & 116 & 98 & 97 & 99 & 99 & 92 & 11.433 & 11.104 & 11.024 \\
\hline $\begin{array}{r}2 \mathrm{M} 08512935 \\
+1145275\end{array}$ & 33.14 & 0.06 & -10.74 & 0.07 & -2.98 & 0.04 & 837.93 & 27.28 & 135 & 98 & 98 & 99 & 99 & 95 & 11.287 & 10.864 & 10.754 \\
\hline $\begin{array}{r}2 \mathrm{M} 08513540 \\
+1157564\end{array}$ & 33.39 & 0.05 & -11.10 & 0.07 & -3.01 & 0.04 & 848.14 & 24.64 & 238 & 98 & 96 & 96 & 99 & 95 & 11.447 & 11.143 & 11.030 \\
\hline $\begin{array}{r}2 \mathrm{M} 08513862 \\
+1220141\end{array}$ & 33.74 & 0.12 & -10.95 & 0.08 & -3.00 & 0.05 & 858.14 & 32.96 & 251 & 98 & 25 & 99 & 97 & 93 & 11.298 & 10.866 & 10.791 \\
\hline $\begin{array}{r}2 \mathrm{M} 08514401 \\
+1146245\end{array}$ & 33.11 & 0.13 & -11.10 & 0.07 & -2.89 & 0.05 & 870.91 & 29.12 & 116 & 95 & 99 & 99 & 98 & 95 & 11.438 & 11.110 & 11.027 \\
\hline $\begin{array}{r}2 \mathrm{M} 08514474 \\
+1146460\end{array}$ & 33.12 & 0.06 & -11.06 & 0.07 & -3.12 & 0.04 & 798.50 & 23.63 & 351 & 98 & 100 & 99 & 98 & 92 & 11.357 & 10.918 & 10.822 \\
\hline $\begin{array}{r}2 \mathrm{M} 08514994 \\
+1149311\end{array}$ & 33.33 & 0.13 & -11.35 & 0.07 & -3.10 & 0.04 & 858.19 & 27.21 & 205 & 98 & 99 & 99 & 99 & 0 & 11.494 & 11.196 & 11.148 \\
\hline $\begin{array}{r}2 \mathrm{M} 08515335 \\
+1148208\end{array}$ & 34.28 & 0.04 & -11.44 & 0.07 & -2.94 & 0.04 & 817.06 & 26.68 & 189 & 98 & 99 & 7. & nan & 99 & 90 & 11.625 & 11.390 \\
\hline $\begin{array}{r}2 \mathrm{M} 08521134 \\
+1145380\end{array}$ & 33.05 & 0.04 & -10.98 & 0.07 & -2.99 & 0.04 & 858.43 & 27.32 & 113 & 98 & 98 & 99 & 37 & 0 & 11.452 & 11.082 & 10.993 \\
\hline
\end{tabular}


Table 1

(Continued)

\begin{tabular}{|c|c|c|c|c|c|c|c|c|c|c|c|c|c|c|c|c|c|}
\hline 2Mass ID & $\begin{array}{c}\mathrm{RV} \\
\left(\mathrm{km} \mathrm{s}^{-1}\right)\end{array}$ & $\begin{array}{c}\sigma(\mathrm{RV}) \\
\sigma(\mathrm{km} \\
\left.\mathrm{s}^{-1}\right)\end{array}$ & $\begin{array}{l}\text { PM (R.A.) } \\
\left(\mu_{\alpha} \cos (\delta)\right)\end{array}$ & $\begin{array}{c}\text { PM } \\
\text { (R.A.) } \\
\quad \sigma\end{array}$ & $\begin{array}{c}\text { PM } \\
\text { (Decl.) } \\
\left(\mu_{\delta}\right)\end{array}$ & $\begin{array}{c}\text { PM } \\
\text { (Decl.) } \\
\\
\sigma\end{array}$ & $\begin{array}{c}\text { Dist BJ18 } \\
\text { parsec }\end{array}$ & $\begin{array}{l}\text { Dist }(\sigma) \\
\text { parsec }\end{array}$ & $\mathrm{S} / \mathrm{N}$ & $\begin{array}{l}\text { Prob } \\
\text { G15 }\end{array}$ & $\begin{array}{l}\text { Prob } \\
\text { Y08 }\end{array}$ & $\begin{array}{l}\text { Prob } \\
\text { Z93 }\end{array}$ & $\begin{array}{l}\text { Prob } \\
\text { G89 }\end{array}$ & $\begin{array}{l}\text { Prob } \\
\text { S77 }\end{array}$ & $J$ & $H$ & $K_{s}$ \\
\hline $\begin{array}{r}\text { 2M08503667 } \\
+1148553\end{array}$ & 35.36 & 0.22 & -11.43 & 0.06 & -3.11 & 0.04 & 899.99 & 30.68 & 162 & 97 & 99 & 99 & 98 & 96 & 11.930 & 11.628 & 11.578 \\
\hline $\begin{array}{r}2 \mathrm{M} 08505569 \\
+1152146\end{array}$ & 34.08 & 0.09 & -11.01 & 0.18 & -2.84 & 0.13 & 930.54 & 57.06 & 425 & 97 & 99 & 99 & 95 & 95 & 10.852 & 10.586 & 10.515 \\
\hline $\begin{array}{r}2 \mathrm{M} 08510106 \\
+1150108\end{array}$ & 32.90 & 0.14 & -10.79 & 0.09 & -2.93 & 0.06 & 899.78 & 30.39 & 117 & 97 & 100 & 93 & 98 & 87 & 11.380 & 11.018 & 10.951 \\
\hline $\begin{array}{r}2 \mathrm{M} 08510951 \\
+1141449\end{array}$ & 32.36 & 0.08 & -10.33 & 0.07 & -3.11 & 0.06 & 897.57 & 30.12 & 113 & 97 & 100 & 99 & 47 & 0 & 11.445 & 11.102 & 10.997 \\
\hline $\begin{array}{r}2 \mathrm{M} 08511877 \\
+1151186\end{array}$ & 34.07 & 0.10 & -10.98 & 0.07 & -2.74 & 0.05 & 883.67 & 33.72 & 333 & 98 & 0.0 & 99 & 99 & 95 & 11.502 & 11.089 & 11.020 \\
\hline $\begin{array}{r}2 \mathrm{M} 08515567 \\
+1217573\end{array}$ & 33.52 & 0.05 & -10.99 & 0.08 & -2.86 & 0.06 & 935.73 & 37.02 & 226 & 96 & 100 & 99 & 98 & 96 & 11.516 & 11.115 & 11.005 \\
\hline Turnoff & & & & & & & & & & & & & & & & & \\
\hline $\begin{array}{r}2 \mathrm{M} 08503392 \\
+1146272\end{array}$ & 33.78 & 0.14 & -10.97 & 0.08 & -3.05 & 0.06 & 869.53 & 32.54 & 241 & 98 & 99 & 99 & 99 & 96 & 11.824 & 11.596 & 11.517 \\
\hline $\begin{array}{r}2 \mathrm{M} 08504079 \\
+1147462\end{array}$ & 34.59 & 0.06 & -10.89 & 0.07 & -3.08 & 0.05 & 847.32 & 28.41 & 170 & 98 & 99 & 86 & 98 & 93 & 11.793 & 11.540 & 11.498 \\
\hline $\begin{array}{r}2 \mathrm{M} 08505177 \\
+1200247\end{array}$ & 33.72 & 0.25 & -11.22 & 0.05 & -2.85 & 0.05 & 867.93 & 21.00 & 146 & 75 & 99 & 16 & 94 & 95 & 12.377 & 12.106 & 12.051 \\
\hline $\begin{array}{r}2 \mathrm{M} 08505702 \\
+1159158\end{array}$ & 33.96 & 0.22 & -11.06 & 0.05 & -3.72 & 0.03 & 840.73 & 21.18 & 178 & 98 & 100 & 99 & 98 & 94 & 12.003 & 11.726 & 11.673 \\
\hline $\begin{array}{r}2 \mathrm{M} 08505762 \\
+1155147\end{array}$ & 33.07 & 0.24 & -10.71 & 0.04 & -2.85 & 0.03 & 870.75 & 17.25 & 148 & 98 & 100 & 94 & 98 & 93 & 12.294 & 12.038 & 11.973 \\
\hline $\begin{array}{r}2 \mathrm{M} 08505903 \\
+1148576\end{array}$ & 33.67 & 0.44 & -10.97 & 0.05 & -2.73 & 0.03 & 867.89 & 21.19 & 118 & 94 & 100 & 99 & 99 & 96 & 12.386 & 12.206 & 12.094 \\
\hline $\begin{array}{r}2 \mathrm{M} 08505973 \\
+1139524\end{array}$ & 33.21 & 0.19 & -10.62 & 0.07 & -2.74 & 0.05 & 831.18 & 28.29 & 104 & 98 & 100 & 99 & 99 & 96 & 12.025 & 11.735 & 11.703 \\
\hline $\begin{array}{r}\text { M08510969 } \\
+1159096\end{array}$ & 33.98 & 7.27 & -10.79 & 0.04 & -2.93 & 0.03 & 857.36 & 14.81 & 154 & 98 & 99 & 99 & 72 & 94 & 12.658 & 12.348 & 12.298 \\
\hline $\begin{array}{r}2 \mathrm{M} 08511576 \\
+1152587\end{array}$ & 35.82 & 0.08 & -11.96 & 0.07 & -2.03 & 0.05 & 844.46 & 29.65 & 162 & 98 & 99 & 99 & 99 & 64 & 11.728 & 11.453 & 11.391 \\
\hline $\begin{array}{r}2 \mathrm{M} 08512240 \\
+1151291\end{array}$ & 33.44 & 0.16 & -10.94 & 0.05 & -2.96 & 0.04 & 853.50 & 20.51 & 132 & 98 & 99 & 94 & 84 & 79 & 12.195 & 11.952 & 11.862 \\
\hline $\begin{array}{r}2 \mathrm{M} 08513710 \\
+1154599\end{array}$ & 34.85 & 0.05 & -10.85 & 0.04 & -2.95 & 0.03 & 858.09 & 19.44 & 127 & 96 & 99 & 99 & 0 & 0 & 12.096 & 11.819 & 11.763 \\
\hline $\begin{array}{r}2 \mathrm{M} 08513806 \\
+1201243\end{array}$ & 32.14 & 0.12 & -11.03 & 0.06 & -3.40 & 0.04 & 839.80 & 28.69 & 144 & 98 & 99 & 99 & 99 & 69 & 11.844 & 11.551 & 11.495 \\
\hline $\begin{array}{r}2 \mathrm{M} 08514122 \\
+1154290\end{array}$ & 33.61 & 0.21 & -11.15 & 0.07 & -3.06 & 0.05 & 820.44 & 25.67 & 213 & 83 & 98 & 99 & 81 & 94 & 11.703 & 11.466 & 11.397 \\
\hline $\begin{array}{r}2 \mathrm{M} 08514475 \\
+1145012\end{array}$ & 34.89 & 0.20 & -10.87 & 0.04 & -2.81 & 0.03 & 855.48 & 18.41 & 136 & 98 & 97 & 99 & 99 & 95 & 12.288 & 12.039 & 11.969 \\
\hline $\begin{array}{r}2 \mathrm{M} 08520741 \\
+1150221\end{array}$ & 34.19 & 0.16 & -11.12 & 0.04 & -2.95 & 0.03 & 864.00 & 18.90 & 202 & 98 & 99 & 99 & 77 & 83 & 12.097 & 11.823 & 11.806 \\
\hline
\end{tabular}


Table 1

(Continued)

\begin{tabular}{|c|c|c|c|c|c|c|c|c|c|c|c|c|c|c|c|c|c|}
\hline 2Mass ID & $\begin{array}{c}\mathrm{RV} \\
\left(\mathrm{km} \mathrm{s}^{-1}\right)\end{array}$ & $\begin{array}{c}\sigma(\mathrm{RV}) \\
\sigma(\mathrm{km} \\
\left.\mathrm{s}^{-1}\right)\end{array}$ & $\begin{array}{l}\text { PM (R.A.) } \\
\left(\mu_{\alpha} \cos (\delta)\right)\end{array}$ & $\begin{array}{c}\text { PM } \\
\text { (R.A.) } \\
\\
\sigma\end{array}$ & $\begin{array}{c}\text { PM } \\
\text { (Decl.) } \\
\left(\mu_{\delta}\right)\end{array}$ & $\begin{array}{c}\text { PM } \\
\text { (Decl.) } \\
\\
\sigma\end{array}$ & $\begin{array}{c}\text { Dist BJ18 } \\
\text { parsec }\end{array}$ & $\begin{array}{l}\text { Dist }(\sigma) \\
\text { parsec }\end{array}$ & $\mathrm{S} / \mathrm{N}$ & $\begin{array}{l}\text { Prob } \\
\text { G15 }\end{array}$ & $\begin{array}{l}\text { Prob } \\
\text { Y08 }\end{array}$ & $\begin{array}{l}\text { Prob } \\
\text { Z93 }\end{array}$ & $\begin{array}{l}\text { Prob } \\
\text { G89 }\end{array}$ & $\begin{array}{l}\text { Prob } \\
\text { S77 }\end{array}$ & $J$ & $H$ & $K_{s}$ \\
\hline \multicolumn{18}{|l|}{ Main Sequence } \\
\hline $\begin{array}{r}2 \mathrm{M} 08502805 \\
+1154505\end{array}$ & 34.95 & 0.20 & -10.55 & 0.04 & -2.41 & 0.03 & 860.00 & 19.59 & 122 & 98 & 100 & 99 & 99 & 93 & 12.968 & 12.665 & 12.563 \\
\hline $\begin{array}{r}2 \mathrm{M} 08511229 \\
+1154230\end{array}$ & 35.13 & 0.26 & -10.81 & 0.05 & -2.87 & 0.04 & 850.83 & 21.65 & 118 & 98 & 97 & 99 & 98 & 91 & 12.986 & 12.708 & 12.623 \\
\hline $\begin{array}{r}2 \mathrm{M} 08512314 \\
+1154049\end{array}$ & 33.62 & 0.33 & -10.83 & 0.05 & -2.76 & 0.03 & 846.83 & 20.20 & 119 & 98 & 98 & 99 & 93 & 94 & 13.017 & 12.741 & 12.681 \\
\hline $\begin{array}{r}2 \mathrm{M} 08512604 \\
+1149555\end{array}$ & 32.89 & 0.30 & -11.76 & 0.06 & -3.28 & 0.04 & 853.19 & 25.88 & 137 & 98 & 97 & 99 & 98 & 92 & 13.344 & 12.987 & 12.897 \\
\hline $\begin{array}{r}2 \mathrm{M} 08512996 \\
+1151090\end{array}$ & 34.82 & 0.23 & -11.07 & 0.05 & -3.08 & 0.03 & 855.38 & 22.12 & 192 & 98 & 99 & 89 & 99 & 93 & 12.926 & 12.630 & 12.599 \\
\hline $\begin{array}{r}2 \mathrm{M} 08513119 \\
+1153179\end{array}$ & 34.17 & 0.30 & -10.82 & 0.04 & -2.98 & 0.03 & 858.47 & 19.64 & 156 & 98 & 99 & 99 & 97 & 95 & 12.603 & 12.327 & 12.267 \\
\hline $\begin{array}{r}2 \mathrm{M} 08513701 \\
+1136516\end{array}$ & 33.18 & 0.67 & -10.80 & 0.06 & -3.31 & 0.04 & 848.99 & 24.24 & 100 & 98 & 100 & 92 & 98 & 94 & 13.341 & 12.932 & 12.829 \\
\hline $\begin{array}{r}2 \mathrm{M} 08514189 \\
+1149376\end{array}$ & 35.88 & 0.28 & -11.02 & 0.06 & -2.92 & 0.04 & 862.75 & 25.07 & 112 & 98 & 98 & 99 & 98 & 95 & 13.626 & 13.262 & 13.189 \\
\hline $\begin{array}{r}2 \mathrm{M} 08514742 \\
+1147096\end{array}$ & 31.44 & 6.03 & -11.11 & 0.05 & -3.09 & 0.03 & 840.28 & 19.47 & 113 & 98 & 99 & 95 & 99 & 96 & 12.880 & 12.496 & 12.372 \\
\hline $\begin{array}{r}2 \mathrm{M} 08521649 \\
+1147382\end{array}$ & 33.91 & 0.27 & -11.01 & 0.07 & -2.74 & 0.04 & 814.76 & 28.03 & 120 & 98 & 99 & 99 & 99 & 77 & 13.558 & 13.221 & 13.157 \\
\hline $\begin{array}{r}2 \mathrm{M} 08505439 \\
+1156290\end{array}$ & 33.73 & 0.11 & -10.74 & 0.07 & -3.19 & 0.05 & 919.56 & 34.20 & 270 & 98 & 98 & 99 & 99 & 95 & 11.706 & 11.435 & 11.372 \\
\hline $\begin{array}{r}2 \mathrm{M} 08510076 \\
+1153115\end{array}$ & 34.05 & 0.28 & -10.76 & 0.06 & -2.93 & 0.05 & 914.22 & 28.97 & 119 & 96 & 100 & 96 & 97 & 93 & 13.474 & 13.157 & 13.105 \\
\hline $\begin{array}{r}2 \mathrm{M} 08511176 \\
+1150018\end{array}$ & 33.53 & 0.29 & -11.02 & 0.08 & -3.20 & 0.05 & 899.09 & 29.78 & 127 & 3 & 100 & 96 & 98 & 92 & 13.665 & 13.120 & 13.031 \\
\hline $\begin{array}{r}2 \mathrm{M} 08512080 \\
+1145024\end{array}$ & 33.77 & 0.11 & -10.51 & 0.06 & -3.81 & 0.04 & 889.07 & 28.43 & 109 & nan & 89 & 97 & 95 & 99 & 11.928 & 11.679 & 11.603 \\
\hline $\begin{array}{r}2 \mathrm{M} 08512742 \\
+1153265\end{array}$ & 34.28 & 0.18 & -10.87 & 0.06 & -3.09 & 0.04 & 948.69 & 34.44 & 273 & 95 & 96 & 99 & 98 & 95 & 11.667 & 11.382 & 11.342 \\
\hline $\begin{array}{r}2 \mathrm{M} 08512788 \\
+1155409\end{array}$ & 36.06 & 0.18 & -11.13 & 0.04 & -2.40 & 0.03 & 893.36 & 18.68 & 129 & 98 & 100 & 99 & 99 & 95 & 12.168 & 11.831 & 11.813 \\
\hline $\begin{array}{r}2 \mathrm{M} 08513012 \\
+1143498\end{array}$ & 33.53 & 0.23 & -11.11 & 0.09 & -3.09 & 0.06 & 887.79 & 38.35 & 102 & 98 & 1 & 99 & 99 & 99 & 12.011 & 11.761 & 11.694 \\
\hline $\begin{array}{r}2 \mathrm{M} 08513455 \\
+1149068\end{array}$ & 33.53 & 0.41 & -11.05 & 0.08 & -3.20 & 0.05 & 887.52 & 27.47 & 104 & 98 & 91 & 96 & 99 & 95 & 13.717 & 13.229 & 13.121 \\
\hline $\begin{array}{r}2 \mathrm{M} 08521868 \\
+1143246\end{array}$ & 32.73 & 0.18 & -10.97 & 0.07 & -2.86 & 0.04 & 877.95 & 32.18 & 143 & 98 & 99 & 88 & 99 & 75 & 11.590 & 11.352 & 11.259 \\
\hline $\begin{array}{r}2 \mathrm{M} 08512643 \\
+1143506\end{array}$ & 33.45 & 0.26 & -11.37 & 0.11 & -2.69 & 0.08 & 835.57 & 38.90 & 121 & 98 & 100 & 96 & 99 & 95 & 11.020 & 11.011 & 10.993 \\
\hline $\begin{array}{r}2 \mathrm{M} 08513259 \\
+1148520\end{array}$ & 33.74 & 0.27 & -11.30 & 0.08 & -3.11 & 0.05 & 791.70 & 28.99 & 146 & 96 & 98 & 99 & 87 & 0 & 10.645 & 10.541 & 10.526 \\
\hline
\end{tabular}


Table 1

(Continued)

\begin{tabular}{|c|c|c|c|c|c|c|c|c|c|c|c|c|c|c|c|c|c|}
\hline 2Mass ID & $\begin{array}{c}\mathrm{RV} \\
\left(\mathrm{km} \mathrm{s}^{-1}\right)\end{array}$ & $\begin{array}{c}\sigma(\mathrm{RV}) \\
\sigma(\mathrm{km} \\
\left.\mathrm{s}^{-1}\right)\end{array}$ & $\begin{array}{l}\text { PM (R.A.) } \\
\left(\mu_{\alpha} \cos (\delta)\right)\end{array}$ & $\begin{array}{c}\text { PM } \\
\text { (R.A.) } \\
\\
\sigma\end{array}$ & $\begin{array}{c}\text { PM } \\
(\text { Decl. }) \\
\left(\mu_{\delta}\right)\end{array}$ & $\begin{array}{c}\text { PM } \\
\text { (Decl.) } \\
\quad \sigma\end{array}$ & $\begin{array}{c}\text { Dist BJ18 } \\
\text { parsec }\end{array}$ & $\begin{array}{l}\text { Dist }(\sigma) \\
\text { parsec }\end{array}$ & $\mathrm{S} / \mathrm{N}$ & $\begin{array}{l}\text { Prob } \\
\text { G15 }\end{array}$ & $\begin{array}{l}\text { Prob } \\
\text { Y08 }\end{array}$ & $\begin{array}{l}\text { Prob } \\
\text { Z93 }\end{array}$ & $\begin{array}{l}\text { Prob } \\
\text { G89 }\end{array}$ & $\begin{array}{l}\text { Prob } \\
\text { S77 }\end{array}$ & $J$ & $H$ & $K_{s}$ \\
\hline \multicolumn{18}{|c|}{$\begin{array}{l}\text { Excluded sample due } \\
\text { to low } \mathrm{S} / \mathrm{N}(<100) \\
\text { Subgiant }\end{array}$} \\
\hline $\begin{array}{r}2 \mathrm{M} 08503438 \\
+1139566\end{array}$ & 33.77 & 0.21 & -10.79 & 0.07 & -2.94 & 0.05 & 850.43 & 27.79 & 99 & 98 & 99 & 91 & 98 & 96 & 11.513 & 11.244 & 11.177 \\
\hline $\begin{array}{r}2 \mathrm{M} 08504198 \\
+1136525\end{array}$ & 34.46 & 0.10 & -11.12 & 0.07 & -3.10 & 0.05 & 852.64 & 27.51 & 91 & 99 & 99 & 99 & 99 & 0 & 11.410 & 11.062 & 10.998 \\
\hline $\begin{array}{r}2 \mathrm{M} 08510811 \\
+1201065\end{array}$ & 33.83 & 0.23 & -11.70 & 0.04 & -3.02 & 0.03 & 875.47 & 20.06 & 99 & 98 & 96 & 99 & 99 & 95 & 12.469 & 12.159 & 12.073 \\
\hline $\begin{array}{r}2 \mathrm{M} 08511826 \\
+1150196\end{array}$ & 34.28 & 4.21 & -10.89 & 0.06 & -2.59 & 0.04 & 863.61 & 27.89 & 86 & 98 & 99 & 94 & 98 & 96 & 13.042 & 12.680 & 12.592 \\
\hline $\begin{array}{r}2 \mathrm{M} 08520356 \\
+1141238\end{array}$ & 34.15 & 0.07 & -10.82 & 0.07 & -2.76 & 0.04 & 852.79 & 27.94 & 99 & 98 & 99 & 99 & 99 & 89 & 11.634 & 11.365 & 11.306 \\
\hline \multicolumn{18}{|l|}{ Main Sequence } \\
\hline $\begin{array}{r}2 \mathrm{M} 08502833 \\
+1142097\end{array}$ & 33.75 & 0.39 & -10.85 & 0.07 & -2.83 & 0.05 & 832.76 & 25.30 & 64 & 98 & 97 & 99 & 98 & 88 & 11.899 & 11.654 & 11.587 \\
\hline $\begin{array}{r}2 \mathrm{M} 08503788 \\
+1252295\end{array}$ & 32.37 & 0.30 & -11.83 & 0.07 & -3.44 & 0.05 & 801.51 & 25.81 & 99 & nan & nan & nan & nan & nan & 13.662 & 13.239 & 13.139 \\
\hline $\begin{array}{r}2 \mathrm{M} 08505334 \\
+1143399\end{array}$ & 32.72 & 0.33 & -10.89 & 0.05 & -3.92 & 0.04 & 841.16 & 21.35 & 89 & 98 & 100 & 99 & 98 & 94 & 13.058 & 12.746 & 12.628 \\
\hline $\begin{array}{r}2 \mathrm{M} 08505923 \\
+1146129\end{array}$ & 31.98 & 1.78 & -10.90 & 0.05 & -2.84 & 0.03 & 810.49 & 18.26 & 55 & 98 & 100 & 99 & 98 & 96 & 12.271 & 11.998 & 11.934 \\
\hline $\begin{array}{r}2 \mathrm{M} 08512386 \\
+1138521\end{array}$ & 34.61 & 0.35 & -11.04 & 0.06 & -2.81 & 0.05 & 849.79 & 23.97 & 89 & 98 & 99 & 99 & 80 & 95 & 13.313 & 12.952 & 12.942 \\
\hline $\begin{array}{r}2 \mathrm{M} 08513215 \\
+1136126\end{array}$ & 34.34 & 0.41 & -11.22 & 0.04 & -2.83 & 0.03 & 869.31 & 17.26 & 52 & 94 & 96 & 99 & 99 & 89 & 12.207 & 11.965 & 11.910 \\
\hline $\begin{array}{r}2 \mathrm{M} 08513444 \\
+1137574\end{array}$ & 34.02 & 0.12 & -10.78 & 0.04 & -2.66 & 0.03 & 829.37 & 17.97 & 58 & 98 & 97 & 99 & 67 & 1 & 12.102 & 11.864 & 11.778 \\
\hline $\begin{array}{r}2 \mathrm{M} 08514375 \\
+1145148\end{array}$ & 32.40 & 0.19 & -11.22 & 0.06 & -2.94 & 0.04 & 848.12 & 24.69 & 55 & 98 & 97 & 99 & 99 & 95 & 12.027 & 11.805 & 11.729 \\
\hline $\begin{array}{r}2 \mathrm{M} 08514465 \\
+1141510\end{array}$ & 32.95 & 0.27 & -11.33 & 0.04 & -2.95 & 0.03 & 860.46 & 17.58 & 67 & 97 & 99 & 99 & 98 & 95 & 12.120 & 11.887 & 11.802 \\
\hline $\begin{array}{r}2 \mathrm{M} 08515290 \\
+1146358\end{array}$ & 34.00 & 0.53 & -11.09 & 0.09 & -2.78 & 0.05 & 865.42 & 23.43 & 97 & 98 & 94 & 99 & 99 & 94 & 13.961 & 13.429 & 13.282 \\
\hline $\begin{array}{r}2 \mathrm{M} 08521664 \\
+1142300\end{array}$ & 32.23 & 3.99 & -10.94 & 0.05 & -2.95 & 0.03 & 805.89 & 17.43 & 70 & 98 & 96 & 99 & 97 & 92 & 12.403 & 12.144 & 12.104 \\
\hline $\begin{array}{r}2 \mathrm{M} 08504511 \\
+1136023\end{array}$ & 31.15 & 13.33 & -10.81 & 0.08 & -2.70 & 0.07 & 842.68 & 17.27 & 76 & 97 & 99 & 99 & 98 & 71 & 13.800 & 13.210 & 13.123 \\
\hline $\begin{array}{r}2 \mathrm{M} 08510131 \\
+1141587\end{array}$ & 32.07 & 10.61 & -11.04 & 0.04 & -2.81 & 0.03 & 879.70 & 20.72 & 80 & 96 & 100 & 99 & 98 & 95 & 12.420 & 12.167 & 12.075 \\
\hline $\begin{array}{r}2 \mathrm{M} 08510156 \\
+1147501\end{array}$ & 32.93 & 0.23 & -10.89 & 0.05 & -3.60 & 0.04 & 876.51 & 23.19 & 57 & 98 & 100 & 93 & 98 & 96 & 12.371 & 12.067 & 11.991 \\
\hline $\begin{array}{r}2 \mathrm{M} 08511229 \\
+1146212\end{array}$ & 31.51 & 0.28 & -10.93 & 0.05 & -3.04 & 0.04 & 919.79 & 20.28 & 73 & 98 & 99 & 93 & 91 & 91 & 12.060 & 11.751 & 11.704 \\
\hline
\end{tabular}


Table 1

(Continued)

\begin{tabular}{|c|c|c|c|c|c|c|c|c|c|c|c|c|c|c|c|c|c|}
\hline 2Mass ID & $\begin{array}{c}\mathrm{RV} \\
\left(\mathrm{km} \mathrm{s}^{-1}\right)\end{array}$ & $\begin{array}{c}\sigma(\mathrm{RV}) \\
\sigma(\mathrm{km} \\
\left.\mathrm{s}^{-1}\right)\end{array}$ & $\begin{array}{l}\text { PM (R.A.) } \\
\left(\mu_{\alpha} \cos (\delta)\right)\end{array}$ & $\begin{array}{c}\text { PM } \\
\text { (R.A.) } \\
\\
\sigma\end{array}$ & $\begin{array}{c}\text { PM } \\
(\text { Decl.) } \\
\left(\mu_{\delta}\right)\end{array}$ & $\begin{array}{c}\text { PM } \\
\text { (Decl.) } \\
\sigma\end{array}$ & $\begin{array}{c}\text { Dist BJ18 } \\
\text { parsec }\end{array}$ & $\begin{array}{l}\text { Dist }(\sigma) \\
\text { parsec }\end{array}$ & $\mathrm{S} / \mathrm{N}$ & $\begin{array}{l}\text { Prob } \\
\text { G15 }\end{array}$ & $\begin{array}{l}\text { Prob } \\
\text { Y08 }\end{array}$ & Z93 & G89 & $\begin{array}{l}\text { Prob } \\
\text { S77 }\end{array}$ & $J$ & $H$ & $K_{s}$ \\
\hline $\begin{array}{r}2 \mathrm{M} 08511810 \\
+1142547\end{array}$ & 33.88 & 0.11 & -10.96 & 0.05 & -2.89 & 0.04 & 923.46 & 25.50 & 97 & 99 & 99 & 0 & 84 & 94 & 12.186 & 11.879 & 11.844 \\
\hline $\begin{array}{r}2 \mathrm{M} 08512033 \\
+1145523\end{array}$ & 33.66 & 0.29 & -10.89 & 0.06 & -2.96 & 0.04 & 876.62 & 24.68 & 76 & 98 & 84 & 99 & 99 & 94 & 12.061 & 11.822 & 11.767 \\
\hline $\begin{array}{r}2 \mathrm{M} 08512176 \\
+1144050\end{array}$ & 32.79 & 0.46 & -11.28 & 0.05 & -3.12 & 0.04 & 881.45 & 24.05 & 60 & 96 & 99 & 96 & 97 & 96 & 12.907 & 12.547 & 12.498 \\
\hline $\begin{array}{r}2 \mathrm{M} 08512467 \\
+1143061\end{array}$ & 32.01 & 0.32 & -10.88 & 0.07 & -2.18 & 0.05 & 926.54 & 36.75 & 82 & 98 & 99 & 99 & 83 & 94 & 13.258 & 12.863 & 12.806 \\
\hline $\begin{array}{r}2 \mathrm{M} 08513424 \\
+1145535\end{array}$ & 34.19 & 0.46 & -10.83 & 0.07 & -2.81 & 0.05 & 941.80 & 35.23 & 77 & 98 & 4 & 99 & 99 & 95 & 13.374 & 12.976 & 12.852 \\
\hline
\end{tabular}

Note. Proper motions and distances from Gaia DR2. 
distance limits, where $\mu_{\alpha} \cos (\delta)=-11.02 \pm 0.07 \mathrm{mas} \mathrm{yr}^{-1}$

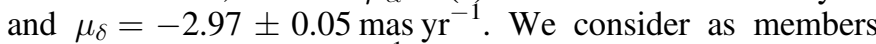

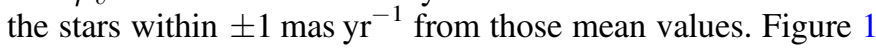
bottom left panel displays the proper motions for the sample.

We removed from the sample two hot stars (2M08512643+ 1143506 and $2 \mathrm{M} 08513259+1148520)$ likely to be blue stragglers. In the final sample, we will only retain the stars with Gaia DR2 data, confirming the membership criteria based on distances and proper motions. We searched for binary stars in our sample looking for RV variations in the multiple spectral visits, with none found. Also, we verify the lack of binary stars in comparing our sample (44 stars in common) with the recent work of El-Badry et al. (2018), where the authors detected more than 3000 binary stars in the APOGEE data. To ensure the quality of the observed spectra, we keep only those having $\mathrm{S} / \mathrm{N} \geqslant 100$, resulting in a sample of 83 stars spanning the H-R diagram, from the main sequence to the red clump. The threshold in $\mathrm{S} / \mathrm{N}$ is intended to minimize the uncertainties in the parameters derived. As we are searching for small abundance variations across the H-R diagram, we assemble the best possible sample. We will include the results reported by Souto et al. (2018) as a control or comparison sample. In Table 1 we present our sample, with the adopted radial velocity and S/N (from DR14), proper motions and distances (Gaia Collaboration et al. 2018), membership probabilities computed by Geller et al. (2015) and the adopted magnitudes, $V$ (Zacharias et al. 2015), and 2MASS infrared magnitudes $J, H$, and $K_{S}$ (Skrutskie et al. 2006). At the bottom of the table, we also provide data for those stars with $\mathrm{S} / \mathrm{N}<100$.

In the top right and bottom right panels of Figure 1, we display the color-magnitude diagram $(\mathrm{CMD})\left(J-K_{S}\right)_{0}$ versus $H_{0}$ and $\left(G_{\mathrm{BP}}-G_{\mathrm{RP}}\right)_{0}$ versus $G_{0}$ for the studied sample using 2MASS and Gaia DR2 photometry, respectively. We show all 563 stars observed in the M67 field by the APOGEE survey with orange dots. Our sample stars are shown as filled symbols, and the ones with $\mathrm{S} / \mathrm{N}<100$ as empty symbols. We note that four early $\mathrm{G}$ and $\mathrm{K}$ dwarfs show a small offset compared to the adopted isochrones presented in the CMD diagrams of Figure 1, which could indicate nonmembership; however, we opt to use these stars because their RVs, proper motions, and distances suggest membership. The same symbol notation adopted by Souto et al. (2018) was used in this work, where diamonds correspond to main sequence, squares to turnoff stars, triangles for subgiants, and the circles represent the red giant stars, in blue for this work and red for Souto et al. (2018).

In Figure 2, we display a portion of the observed APOGEE spectra between 16150 and $16260 \AA$ for the sample stars. From top to bottom, we plot the spectra of the red giant stars followed by the subgiant, turnoff, and main-sequence stars. The individual stellar spectra are very similar within a class, with rms differences at any given wavelength of about $\sigma=0.01$. The largest star-to-star differences in Figure 2 are associated with $\mathrm{CO}, \mathrm{CN}$, and $\mathrm{OH}$ lines in the red giant spectra, suggestive of the changes produced by $\mathrm{H}$ burning in the stellar interior brought to the surface by the first dredge-up, as discussed in Section 5. Fe I and $\mathrm{Ca}$ I show the largest spread among G-type stars.

\section{Stellar Parameters and Chemical Abundances}

In this paper, we need to determine abundances in different classes of stars (dwarfs to red giants) homogeneously and precisely. One important factor in such analysis is the determination of the stellar parameters. It is known that the raw $\log g$ values derived using the ASPCAP pipeline contain systematic offsets for dwarfs (being systematically low) as well as red giant stars (being systematically high).

Figure 3 shows the effective temperature and surface gravity diagrams for our sample. The left panel shows the DR14 raw ASPCAP $T_{\text {eff }}$ and $\log g$ results. It is clear that the $\log g$ values derived by ASPCAP do not match the isochrones from Bressan et al. (2012) and Choi et al. (2016) (presented in the figure). Using such $\log g$ values in the analysis would introduce systematic uncertainties in the derived abundances. In the next section, we discuss the determination of the $\log g$ 's and adopted $T_{\text {eff }}$ values in this study.

\subsection{Effective Temperatures}

We adopted the effective temperatures derived from ASPCAP DR14. We used the purely spectroscopic raw $T_{\text {eff }}$ values from ASPCAP (given in the FPARAM array in DR14). For comparison, we also determined photometric temperatures by adopting the calibration of González-Hernández \& Bonifacio (2009) and using five different colors, $B-V, V-J, V-H, V-K_{s}$, and $J-K_{s}$, with an adopted cluster reddening of $E(B-V)=$ $0.041 \mathrm{mag}$ (Sarajedini et al. 2009) and a metallicity of [Fe/ $\mathrm{H}]=0.00$. González-Hernández \& Bonifacio (2009) provide photometric calibrations for red giant and dwarf stars; we adopted the coefficients for giants for those stars with $\log g<4.00$ dex and for dwarfs for those stars with higher gravities. Good agreement between the photometric and the adopted raw ASPCAP $T_{\text {eff }}$ scales is obtained, where $\left\langle\delta\left(T_{\text {eff }}(\mathrm{ASPCAP}-\mathrm{GHB})\right\rangle=-25 \pm 106 \mathrm{~K}\right.$. The effective temperatures obtained from the ASPCAP pipeline have an internal precision of $\pm 50 \mathrm{~K}$ (Holtzman et al. 2015, García Pérez et al. 2016).

\subsection{Surface Gravities}

We determined surface gravities from the fundamental Equation (1), where the adopted $T_{\text {eff }}$ values are from the raw ASPCAP DR14 values, with stellar masses and bolometric magnitudes obtained from interpolation in the MIST isochrones (Choi et al. 2016; $[\mathrm{Fe} / \mathrm{H}]=0.00$; age $=4.00 \mathrm{Gyr} ; E(B-V)=$ 0.041 ; distance modulus $(\mu)=9.60)$. The adopted solar values are $\log g_{\odot}=4.438 \mathrm{dex}, T_{\text {eff, } \odot}=5772 \mathrm{~K}$, and $M_{\text {bol }, \odot}=4.75$, following the IAU recommendations in Prša et al. (2016):

$$
\begin{aligned}
\log g= & \log _{10} g_{\odot}+\log _{10}\left(\frac{M_{\star}}{M_{\odot}}\right) \\
& +4 \log _{10}\left(\frac{T_{\star}}{T_{\odot}}\right)+0.4\left(M_{\mathrm{bol}, \star}-M_{\mathrm{bol}, \odot}\right) .
\end{aligned}
$$

We adopted the surface gravities derived from Equation (1) in the abundance analysis in this study. The uncertainties in the determined surface gravities are similar to the ones reported in Souto et al. (2018), where $\sigma= \pm 0.10$ dex. The comparison between the derived $\log g$ values in this work with those from ASPCAP confirms the $\log g$ offset, where we obtain $\langle\delta(\log g$ $($ Physical-ASPCAP $)\rangle=-0.18 \pm 0.16 \mathrm{dex}$ for red giants, $-0.16 \pm 0.11$ dex for subgiants, $-0.19 \pm 0.07$ dex for turnoff, and $0.17 \pm 0.13$ dex for main-sequence stars. 

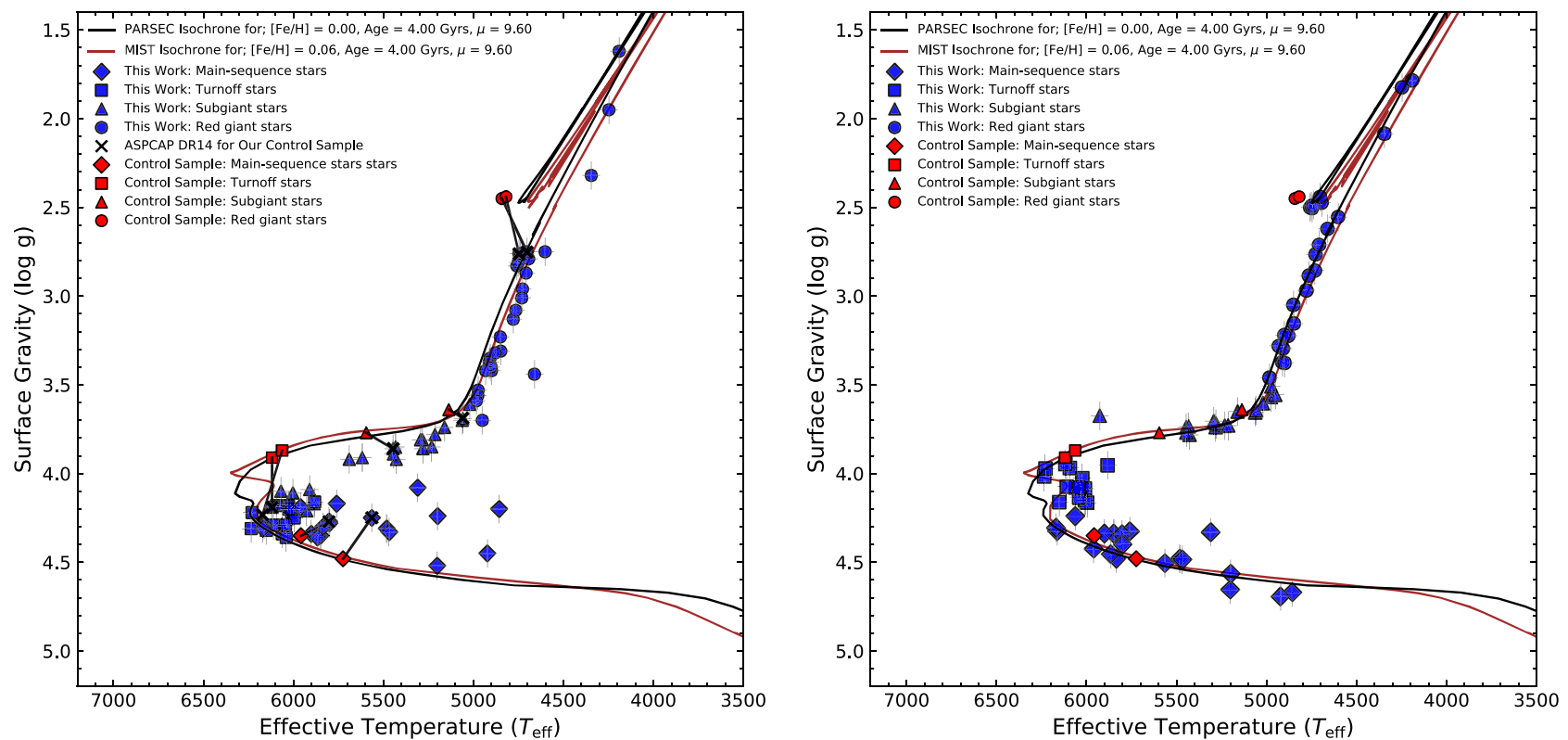

Figure 3. Left panel: $T_{\text {eff }} \log g$ diagram showing the APOGEE DR14 raw ASPCAP results for the M67 members. Note the mismatch with the isochrones due to systematic uncertainties in the $\log g$ values derived by ASPCAP. Right panel: $T_{\text {eff }}-\log g$ diagram showing the stellar parameters adopted in this study. The $T_{\text {eff }}$ values are the same raw values from ASPCAP DR14 shown in the left panel, but the surface gravities were derived from fundamental relations. The symbol notation is the same as in Figure 1.

Figure 3 (right panel) shows the $T_{\text {eff }}-\log g$ values adopted in this study. The effective temperatures for the studied stars are well spread out in the H-R diagram, with effective temperatures ranging between 4200 and $6250 \mathrm{~K}$. The surface gravity values for the studied stars span a range in $\log g=$ 1.78-4.71.

\subsection{Individual Abundance Analysis}

In this work, we derive individual abundances for 15 elements: C, N, O, Na, Mg, Al, Si, K, Ca, Ti, V, Cr, Mn, Fe, and $\mathrm{Ni}$. Individual abundances were determined with the qASPCAP code. The qASPCAP code basically corresponds to the ASPCAP pipeline, but for custom work, providing flexibility to change the analysis parameters. The methodology in the analysis is the same as adopted in ASPCAP, and the optimization is based on the FERRE code.

The procedure for determining individual abundances and microturbulent velocities with qASPCAP is similar to the one in ASPCAP. The ASPCAP pipeline (described in detail in García Pérez et al. 2016) uses a grid of synthetic spectra (Zamora et al. 2015) computed with the turbospectrum code (Alvarez \& Plez 1998, Plez 2012) using KURUCZ model atmospheres (Castelli \& Kurucz 2004, Mészáros et al. 2012) and the APOGEE DR14 line list, which is an updated version of the one published in Shetrone et al. (2015). The stellar parameters and chemical abundances are obtained by $\chi^{2}$ minimization with the FERRE code (Allende Prieto et al. 2006) controlled by an IDL wrapper (the qASPCAP in this work).

In a first phase, seven parameters are determined through a 7D optimization $\left(T_{\text {eff }}, \log g,[\mathrm{M} / \mathrm{H}],[\mathrm{C} / \mathrm{Fe}],[\mathrm{N} / \mathrm{Fe}],[\alpha / \mathrm{Fe}]\right.$, and $\xi$ ) using the entire wavelength range of the APOGEE spectra. During the second phase, individual abundances are obtained by repeating the fitting in predetermined windows that are sensitive to elemental abundances using the set of atmospheric parameters determined in the previous phase. It is possible to determine individual abundances for more than 26 elements from the APOGEE spectra; see Holtzman et al. (2018), Hasselquist et al. (2016, for Nd), and Cunha et al. (2017, for Ce). In this work, we adopt the same molecular and atomic lines as Souto et al. (2018) to derive individual abundances (see also Smith et al. 2013 and Souto et al. 2016). Even though Souto et al. (2018) reported $\mathrm{Na}$ and $\mathrm{Cr}$ abundances for main-sequence and turnoff stars, we opt in this work to not present these abundances (for these stellar classes) as the comparisons between the observed and synthesis were not satisfactory due to the weakness of the $\mathrm{Na} \mathrm{I}$ and $\mathrm{Cr}$ I lines.

All M67 targets studied here have similar $v \sin (i)$, in the range $0 \leqslant v \sin (i) \leqslant 7 \mathrm{~km} \mathrm{~s}^{-1}$. In fact, the threshold to detect the star's $v \sin (i)$ from APOGEE spectra is $7-8 \mathrm{~km} \mathrm{~s}^{-1}$. The effect of macroturbulence on the line profiles is similar to that of stellar rotation, and, as an approximation, qASPCAP treats rotation and macroturbulence as a single Gaussian profile.

The stellar parameters adopted in this work are shown in Table 2, with individual abundances presented in Table 3 . The uncertainties in the derived abundances adopted in this work are the same as the ones reported in Table 4 of Souto et al. (2018). We note that, using ASPCAP calibrated abundances, the average $\langle\delta A(E \mathrm{El})\rangle$ between the results derived in this work minus ASPCAP is smaller by 0.10 dex for all elements.

\section{Results}

The individual abundances reported in this work display an elevated scatter (standard deviation of the mean), in particular for nitrogen $(\sim 0.14 \mathrm{dex})$, aluminum $(\sim 0.16 \mathrm{dex})$, and the alpha elements $(\sim 0.15 \mathrm{dex})$. The potassium abundances are the ones showing the smallest scatter, with $\sigma=0.07$ dex. Such significant scatter in M67 stars was also noticed by Bertran de Lis et al. (2016) studying $[\mathrm{O} / \mathrm{Fe}]$ in M67 stars with APOGEE and comparing it with the spread in other clusters. 
Table 2

Stellar Parameters

\begin{tabular}{|c|c|c|c|c|c|c|c|c|}
\hline 2Mass ID & $\begin{array}{c}T_{\text {eff }}(\mathrm{K}) \\
\text { ASPCAP raw }\end{array}$ & $\begin{array}{c}T_{\text {eff }}(\mathrm{K}) \\
\text { ASPCAP calib }\end{array}$ & $\begin{array}{l}T_{\text {eff }}(\mathrm{K}) \\
\text { GB09 }\end{array}$ & $\begin{array}{l}\log g\left(\mathrm{~cm} \mathrm{~s}^{-2}\right) \\
\text { ASPCAP raw }\end{array}$ & $\begin{array}{l}\log g\left(\mathrm{~cm} \mathrm{~s}^{-2}\right) \\
\text { ASPCAP calib }\end{array}$ & $\begin{array}{c}\log g\left(\mathrm{~cm} \mathrm{~s}^{-2}\right) \\
\text { Physical }\end{array}$ & $\begin{array}{c}\text { Mass }\left(M_{\odot}\right) \\
\text { Mass isochrone }\end{array}$ & $\begin{array}{c}\xi\left(\mathrm{km} \mathrm{s}^{-1}\right) \\
\text { ASPCAP raw }\end{array}$ \\
\hline \multicolumn{9}{|l|}{ Red Giants } \\
\hline $2 \mathrm{M} 08492491+1144057$ & 4848.2 & 4893.5 & 4899.0 & 3.31 & 3.17 & 3.15 & 1.32 & 1.14 \\
\hline $2 \mathrm{M} 08503613+1143180$ & 4973.6 & 5023.9 & 5019.9 & 3.53 & 3.41 & 3.51 & 1.31 & 1.24 \\
\hline $2 \mathrm{M} 08504964+1135089$ & 4727.8 & 4774.7 & 4710.9 & 2.96 & 2.80 & 2.77 & 1.33 & 1.33 \\
\hline $2 \mathrm{M} 08511269+1152423$ & 4758.1 & 4805.4 & 4702.4 & 2.83 & 2.49 & 2.50 & 1.33 & 1.45 \\
\hline $2 \mathrm{M} 08511704+1150464$ & 4707.8 & 4757.9 & 4764.2 & 2.87 & 2.71 & 2.71 & 1.33 & 1.42 \\
\hline $2 \mathrm{M} 08511897+1158110$ & 4907.5 & 4956.5 & 4909.9 & 3.35 & 3.22 & 3.30 & 1.32 & 1.11 \\
\hline $2 \mathrm{M} 08512156+1146061$ & 4731.2 & 4776.9 & 4748.2 & 3.01 & 2.85 & 2.86 & 1.33 & 1.35 \\
\hline $2 \mathrm{M} 08512618+1153520$ & 4750.6 & 4798.5 & 4714.2 & 2.81 & 2.48 & 2.49 & 1.33 & 1.40 \\
\hline $2 \mathrm{M} 08512898+1150330$ & 4693.6 & 4741.1 & 4691.2 & 2.79 & 2.46 & 2.47 & 1.34 & 1.43 \\
\hline $2 \mathrm{M} 08512990+1147168$ & 4247.5 & 4302.4 & 4274.0 & 1.95 & 1.68 & 1.82 & 1.34 & 1.47 \\
\hline $2 \mathrm{M} 08513577+1153347$ & 4911.4 & 4959.5 & 4882.8 & 3.37 & 3.24 & 3.29 & 1.32 & 1.19 \\
\hline $2 \mathrm{M} 08513938+1151456$ & 4878.3 & 4927.4 & 4871.1 & 3.32 & 3.20 & 3.22 & 1.32 & 1.20 \\
\hline $2 \mathrm{M} 08514234+1150076$ & 4778.7 & 4825.8 & 4803.5 & 3.13 & 2.99 & 2.97 & 1.33 & 1.27 \\
\hline $2 \mathrm{M} 08514388+1156425$ & 4747.5 & 4795.8 & 4711.9 & 2.76 & 2.44 & 2.50 & 1.33 & 1.64 \\
\hline $2 \mathrm{M} 08514507+1147459$ & 4765.1 & 4812.5 & 4799.6 & 3.08 & 2.93 & 2.88 & 1.33 & 1.35 \\
\hline $2 \mathrm{M} 08514883+1156511$ & 4976.0 & 5027.4 & 5028.8 & 3.56 & 3.44 & 3.57 & 1.31 & 1.25 \\
\hline $2 \mathrm{M} 08515611+1150147$ & 4950.8 & 4994.9 & 4927.9 & 3.70 & 3.56 & 3.56 & 1.31 & 0.92 \\
\hline $2 \mathrm{M} 08515952+1155049$ & 4740.0 & 4789.0 & 4708.4 & 2.76 & 2.45 & 2.49 & 1.33 & 1.51 \\
\hline $2 \mathrm{M} 08521097+1131491$ & 4602.3 & 4649.1 & 4633.6 & 2.75 & 2.56 & 2.55 & 1.33 & 1.38 \\
\hline $2 \mathrm{M} 08521656+1119380$ & 4345.3 & 4394.8 & 4406.8 & 2.32 & 2.09 & 2.08 & 1.35 & 1.39 \\
\hline $2 \mathrm{M} 08521856+1144263$ & 4702.7 & 4750.1 & 4737.3 & 2.75 & 2.44 & 2.44 & 1.35 & 1.49 \\
\hline $2 \mathrm{M} 08522636+1141277$ & 4912.3 & 4962.6 & 4957.1 & 3.39 & 3.26 & 3.37 & 1.31 & 1.30 \\
\hline $2 \mathrm{M} 08525625+1148539$ & 4899.5 & 4944.1 & 4874.3 & 3.42 & 3.28 & 3.38 & 1.31 & 1.29 \\
\hline $2 \mathrm{M} 08534672+1123307$ & 4850.6 & 4899.3 & 4880.3 & 3.23 & 3.11 & 3.05 & 1.32 & 1.10 \\
\hline $2 \mathrm{M} 08493465+1151256$ & 4190.5 & -9999.0 & 4347.6 & 1.62 & -9999. & 1.78 & 1.34 & 0.53 \\
\hline $2 \mathrm{M} 08505816+1152223$ & 4983.5 & 5030.7 & 5021.5 & 3.59 & 3.46 & 3.46 & 1.31 & 1.05 \\
\hline $2 \mathrm{M} 08510723+1153019$ & 4661.0 & -9999.0 & 5335.0 & 3.44 & -9999. & 2.62 & 1.33 & 0.72 \\
\hline $2 \mathrm{M} 08510839+1147121$ & 4901.4 & 4948.9 & 4995.6 & 3.40 & 3.27 & 3.22 & 1.32 & 1.25 \\
\hline $2 \mathrm{M} 08522003+1127362$ & 4932.4 & 4980.3 & 4973.2 & 3.42 & 3.29 & 3.28 & 1.32 & 1.20 \\
\hline \multicolumn{9}{|l|}{ Subgiants } \\
\hline $2 \mathrm{M} 08504994+1149127$ & 5160.9 & 5213.0 & 5196.8 & 3.74 & 3.62 & 3.65 & 1.30 & 0.96 \\
\hline $2 \mathrm{M} 08510325+1145473$ & 5884.4 & 5928.1 & 5932.2 & 4.17 & -9999. & 3.84 & 1.30 & 0.77 \\
\hline $2 \mathrm{M} 08511564+1150561$ & 5282.5 & 5331.6 & 5271.0 & 3.81 & -9999. & 3.74 & 1.30 & 1.04 \\
\hline $2 \mathrm{M} 08511670+1145293$ & 5280.6 & 5335.8 & 5312.0 & 3.86 & -9999. & 3.72 & 1.24 & 0.87 \\
\hline $2 \mathrm{M} 08512122+1145526$ & 5926.6 & 5971.3 & 6019.4 & 4.21 & -9999. & 3.67 & 1.31 & 0.75 \\
\hline $2 \mathrm{M} 08512879+1151599$ & 5617.4 & 5673.6 & 5624.3 & 3.91 & -9999. & 3.82 & 1.29 & 0.78 \\
\hline $2 \mathrm{M} 08512935+1145275$ & 5019.9 & 5069.7 & 5061.7 & 3.61 & 3.49 & 3.60 & 1.31 & 1.16 \\
\hline $2 \mathrm{M} 08513540+1157564$ & 5446.4 & 5497.9 & 5484.9 & 3.86 & -9999. & 3.74 & 1.29 & 0.65 \\
\hline $2 \mathrm{M} 08513862+1220141$ & 5062.5 & 5112.0 & 4995.6 & 3.70 & 3.58 & 3.66 & 1.30 & 0.94 \\
\hline $2 \mathrm{M} 08514401+1146245$ & 5432.7 & 5483.9 & 5507.6 & 3.85 & -9999. & 3.73 & 1.30 & 0.89 \\
\hline $2 \mathrm{M} 08514474+1146460$ & 5058.6 & 5109.2 & 5065.6 & 3.69 & 3.57 & 3.64 & 1.30 & 0.97 \\
\hline $2 \mathrm{M} 08514994+1149311$ & 6003.3 & 6048.3 & 5887.6 & 4.11 & -9999. & 3.85 & 1.28 & 0.83 \\
\hline $2 \mathrm{M} 08515335+1148208$ & 6069.2 & 6114.7 & 5991.7 & 4.10 & -9999. & 3.89 & 1.27 & 1.06 \\
\hline $2 \mathrm{M} 08521134+1145380$ & 5293.2 & 5343.2 & 5326.3 & 3.81 & -9999. & 3.71 & 1.30 & 0.92 \\
\hline $2 \mathrm{M} 08503667+1148553$ & 5689.4 & 5746.5 & 5874.4 & 3.92 & -9999. & 3.82 & 1.29 & 0.68 \\
\hline $2 \mathrm{M} 08505569+1152146$ & 5910.2 & 5954.9 & 5943.0 & 4.09 & -9999. & 3.85 & 1.28 & 0.67 \\
\hline $2 \mathrm{M} 08510106+1150108$ & 5428.1 & 5480.6 & 5540.4 & 3.92 & -9999. & 3.78 & 1.29 & 0.82 \\
\hline $2 \mathrm{M} 08510951+1141449$ & 5445.3 & 5492.3 & 5462.4 & 3.89 & -9999. & 3.77 & 1.29 & 0.84 \\
\hline $2 \mathrm{M} 08511877+1151186$ & 5231.5 & 5277.7 & 5326.9 & 3.85 & -9999. & 3.73 & 1.30 & 0.81 \\
\hline $2 \mathrm{M} 08515567+1217573$ & 5213.3 & 5264.6 & 5215.4 & 3.78 & 3.66 & 3.73 & 1.30 & 0.91 \\
\hline \multicolumn{9}{|l|}{ Turnoff } \\
\hline $2 \mathrm{M} 08503392+1146272$ & 6235.1 & 6279.1 & 6165.5 & 4.31 & -9999. & 4.02 & 1.25 & 0.73 \\
\hline $2 \mathrm{M} 08504079+1147462$ & 6228.8 & 6274.8 & 6156.4 & 4.22 & -9999. & 3.97 & 1.25 & 0.72 \\
\hline $2 \mathrm{M} 08505177+1200247$ & 6009.0 & 6053.3 & 6042.0 & 4.22 & -9999. & 4.15 & 1.21 & 0.62 \\
\hline $2 \mathrm{M} 08505702+1159158$ & 6024.5 & 6069.9 & 6040.1 & 4.20 & -9999. & 4.03 & 1.27 & 0.55 \\
\hline $2 \mathrm{M} 08505762+1155147$ & 6151.3 & 6196.3 & 6044.2 & 4.32 & -9999. & 4.16 & 1.21 & 0.64 \\
\hline $2 \mathrm{M} 08505903+1148576$ & 5996.1 & -9999.0 & 6090.9 & 4.25 & -9999. & 4.17 & 1.21 & 0.66 \\
\hline $2 \mathrm{M} 08505973+1139524$ & 6061.8 & 6107.0 & 5968.9 & 4.34 & -9999. & 4.08 & 1.25 & 0.64 \\
\hline $2 \mathrm{M} 08510969+1159096$ & 6026.4 & 6073.3 & 5957.1 & 4.18 & -9999. & 4.08 & 1.25 & 0.63 \\
\hline $2 \mathrm{M} 08511576+1152587$ & 6093.1 & 6137.2 & 5960.3 & 4.18 & -9999. & 3.97 & 1.27 & 1.24 \\
\hline $2 \mathrm{M} 08512240+1151291$ & 6009.8 & 6056.5 & 6056.2 & 4.20 & -9999. & 4.08 & 1.23 & 0.85 \\
\hline $2 \mathrm{M} 08513710+1154599$ & 6110.2 & 6156.0 & 6052.5 & 4.29 & -9999. & 4.07 & 1.23 & 0.63 \\
\hline
\end{tabular}


Table 2

(Continued)

\begin{tabular}{|c|c|c|c|c|c|c|c|c|}
\hline 2Mass ID & $\begin{array}{c}T_{\text {eff }}(\mathrm{K}) \\
\text { ASPCAP raw }\end{array}$ & $\begin{array}{c}T_{\text {eff }}(\mathrm{K}) \\
\text { ASPCAP calib }\end{array}$ & $\begin{array}{c}T_{\text {eff }}(\mathrm{K}) \\
\text { GB09 }\end{array}$ & $\begin{array}{l}\log g\left(\mathrm{~cm} \mathrm{~s}^{-2}\right) \\
\text { ASPCAP raw }\end{array}$ & $\begin{array}{l}\log g\left(\mathrm{~cm} \mathrm{~s}^{-2}\right) \\
\text { ASPCAP calib }\end{array}$ & $\begin{array}{c}\log g\left(\mathrm{~cm} \mathrm{~s}^{-2}\right) \\
\text { Physical }\end{array}$ & $\begin{array}{l}\text { Mass }\left(M_{\odot}\right) \\
\text { Mass isochrone }\end{array}$ & $\begin{array}{l}\xi\left(\mathrm{km} \mathrm{s}^{-1}\right) \\
\text { ASPCAP raw }\end{array}$ \\
\hline $2 \mathrm{M} 08513806+1201243$ & 5882.2 & 5926.9 & 5845.0 & 4.16 & -9999. & 3.95 & 1.28 & 0.59 \\
\hline $2 \mathrm{M} 08514122+1154290$ & 6118.1 & 6162.7 & 6008.6 & 4.19 & -9999. & 3.95 & 1.28 & 0.74 \\
\hline $2 \mathrm{M} 08514475+1145012$ & 6040.5 & 6086.9 & 6136.3 & 4.36 & -9999. & 4.14 & 1.23 & 0.68 \\
\hline $\begin{array}{l}\text { 2M08520741+1150221 } \\
\text { Main Sequence }\end{array}$ & 6043.8 & 6087.8 & 6057.0 & 4.29 & -9999. & 4.08 & 1.25 & 0.66 \\
\hline $2 \mathrm{M} 08502805+1154505$ & 5759.3 & 5806.5 & 5755.8 & 4.17 & -9999. & 4.33 & 1.14 & 0.61 \\
\hline $2 \mathrm{M} 08511229+1154230$ & 5848.9 & 5892.7 & 5885.6 & 4.35 & -9999. & 4.34 & 1.12 & 0.68 \\
\hline $2 \mathrm{M} 08512314+1154049$ & 5802.4 & 5847.1 & 5886.3 & 4.27 & -9999. & 4.34 & 1.11 & 0.73 \\
\hline $2 \mathrm{M} 08512604+1149555$ & 5310.1 & 5358.8 & 5472.1 & 4.08 & -9999. & 4.33 & 1.00 & 0.61 \\
\hline $2 \mathrm{M} 08512996+1151090$ & 5900.8 & 5945.5 & 5925.8 & 4.34 & -9999. & 4.34 & 1.11 & 0.64 \\
\hline $2 \mathrm{M} 08513119+1153179$ & 6062.9 & 6108.2 & 6021.5 & 4.30 & -9999. & 4.24 & 1.17 & 0.68 \\
\hline $2 \mathrm{M} 08513701+1136516$ & 5201.7 & 5244.9 & 5211.6 & 4.52 & -9999. & 4.65 & 0.81 & 0.86 \\
\hline $2 \mathrm{M} 08514189+1149376$ & 5481.9 & 5525.2 & 5595.9 & 4.31 & -9999. & 4.48 & 1.03 & 0.90 \\
\hline $2 \mathrm{M} 08514742+1147096$ & 5199.1 & 5245.7 & 5226.8 & 4.24 & -9999. & 4.57 & 0.85 & 0.60 \\
\hline $2 \mathrm{M} 08521649+1147382$ & 5467.4 & 5512.1 & 5573.0 & 4.33 & -9999. & 4.49 & 1.01 & 0.72 \\
\hline $2 \mathrm{M} 08505439+1156290$ & 6163.0 & 6207.7 & 5956.9 & 4.29 & -9999. & 4.33 & 1.10 & 0.63 \\
\hline $2 \mathrm{M} 08510076+1153115$ & 5564.3 & 5609.2 & 5798.0 & 4.25 & -9999. & 4.50 & 0.93 & 0.60 \\
\hline $2 \mathrm{M} 08511176+1150018$ & 4857.4 & 4902.6 & 4779.6 & 4.20 & -9999. & 4.67 & 0.75 & 0.57 \\
\hline $2 \mathrm{M} 08512080+1145024$ & 5799.6 & 5845.3 & 5998.8 & 4.28 & -9999. & 4.40 & 0.98 & 0.67 \\
\hline $2 \mathrm{M} 08512742+1153265$ & 6169.5 & 6214.1 & 5998.8 & 4.31 & -9999. & 4.31 & 1.11 & 0.73 \\
\hline $2 \mathrm{M} 08512788+1155409$ & 5834.3 & 5878.5 & 5860.8 & 4.30 & -9999 . & 4.48 & 0.98 & 0.61 \\
\hline $2 \mathrm{M} 08513012+1143498$ & 5866.0 & 5913.1 & 6036.4 & 4.36 & -9999. & 4.45 & 1.00 & 0.62 \\
\hline $2 \mathrm{M} 08513455+1149068$ & 4922.6 & 4966.8 & 4908.2 & 4.45 & -9999. & 4.69 & 0.75 & 0.86 \\
\hline $2 \mathrm{M} 08521868+1143246$ & 5960.3 & 6006.6 & 5970.8 & 4.19 & -9999. & 4.42 & 1.03 & 0.62 \\
\hline $2 \mathrm{M} 08512643+1143506$ & 7985.2 & -9999.0 & 7686.9 & 4.67 & -9999. & 4.53 & 1.21 & 1.55 \\
\hline $2 \mathrm{M} 08513259+1148520$ & 7599.0 & 7647.2 & 7095.6 & 4.38 & -9999. & 4.44 & 1.21 & 2.82 \\
\hline
\end{tabular}

Excluded sample due

to low $\mathrm{S} / \mathrm{N}(<100)$

Subgiant

\begin{tabular}{|c|c|c|c|c|c|c|c|c|}
\hline $2 \mathrm{M} 08503438+1139566$ & 5955.8 & 5998.6 & 5947.6 & 4.11 & -9999 . & 3.87 & 1.28 & 0.67 \\
\hline $2 \mathrm{M} 08504198+1136525$ & 5597.0 & 5647.9 & 5611.6 & 3.92 & -9999. & 3.79 & 1.29 & 0.94 \\
\hline $2 \mathrm{M} 08510811+1201065$ & 5625.6 & 5674.2 & 5679.9 & 4.02 & -9999. & 3.80 & 1.29 & 0.59 \\
\hline $2 \mathrm{M} 08511826+1150196$ & 5458.1 & 5508.6 & 5454.3 & 4.01 & -9999. & 3.77 & 1.29 & 0.58 \\
\hline $2 \mathrm{M} 08520356+1141238$ & 5954.6 & 6000.4 & 5984.7 & 4.07 & -9999. & 3.89 & 1.28 & 0.70 \\
\hline \multicolumn{9}{|l|}{ Main Sequence } \\
\hline $2 \mathrm{M} 08502833+1142097$ & 6164.8 & 6210.0 & 6060.1 & 4.28 & -9999. & 4.33 & 1.10 & 0.86 \\
\hline $2 \mathrm{M} 08503788+1252295$ & 5362.5 & 5405.2 & 5381.2 & 4.39 & -9999. & 4.68 & 0.87 & 1.11 \\
\hline $2 \mathrm{M} 08505334+1143399$ & 5194.7 & 5242.1 & 5536.2 & 4.22 & -9999. & 4.56 & 0.85 & 0.69 \\
\hline $2 \mathrm{M} 08505923+1146129$ & 5567.1 & 5613.8 & 5943.0 & 4.21 & -9999. & 4.51 & 0.93 & 0.71 \\
\hline $2 \mathrm{M} 08512386+1138521$ & 5614.1 & 5659.0 & 5789.1 & 4.47 & -9999. & 4.52 & 0.93 & 0.70 \\
\hline $2 \mathrm{M} 08513215+1136126$ & 5935.4 & 5981.2 & 6132.1 & 4.33 & -9999. & 4.42 & 1.03 & 0.73 \\
\hline $2 \mathrm{M} 08513444+1137574$ & 6000.8 & 6045.6 & 6003.4 & 4.24 & -9999 . & 4.40 & 1.04 & 0.63 \\
\hline $2 \mathrm{M} 08514375+1145148$ & 6056.9 & 6104.1 & 6127.2 & 4.30 & -9999. & 4.38 & 1.06 & 0.66 \\
\hline $2 \mathrm{M} 08514465+1141510$ & 6091.1 & 6136.1 & 6031.7 & 4.39 & -9999. & 4.36 & 1.07 & 0.72 \\
\hline $2 \mathrm{M} 08515290+1146358$ & 4624.6 & 4670.1 & 4633.9 & 4.34 & -9999. & 4.71 & 0.70 & 1.24 \\
\hline $2 \mathrm{M} 08521664+1142300$ & 5985.2 & 6033.1 & 6122.4 & 4.28 & -9999. & 4.36 & 1.04 & 0.58 \\
\hline $2 \mathrm{M} 08504511+1136023$ & 4547.0 & 4593.2 & 4640.2 & 4.17 & -9999. & 4.55 & 0.75 & 0.57 \\
\hline $2 \mathrm{M} 08510131+1141587$ & 5904.2 & 5953.5 & 5906.2 & 4.24 & -9999. & 4.45 & 1.01 & 0.70 \\
\hline $2 \mathrm{M} 08510156+1147501$ & 5642.9 & 5688.3 & 5749.4 & 4.17 & -9999. & 4.49 & 0.95 & 0.58 \\
\hline $2 \mathrm{M} 08511229+1146212$ & 5806.2 & 5851.6 & 5856.3 & 4.25 & -9999. & 4.47 & 0.98 & 0.64 \\
\hline $2 \mathrm{M} 08511810+1142547$ & 5895.8 & 5940.7 & 5920.0 & 4.27 & -9999. & 4.45 & 1.01 & 0.70 \\
\hline $2 \mathrm{M} 08512033+1145523$ & 6063.8 & 6108.9 & 6146.6 & 4.32 & -9999 . & 4.39 & 1.06 & 0.68 \\
\hline $2 \mathrm{M} 08512176+1144050$ & 5360.8 & 5406.7 & 5624.3 & 4.26 & -9999 . & 4.60 & 0.86 & 0.64 \\
\hline $2 \mathrm{M} 08512467+1143061$ & 5219.1 & 5264.6 & 5446.2 & 4.39 & -9999. & 4.57 & 0.85 & 0.78 \\
\hline $2 \mathrm{M} 08513424+1145535$ & 5193.5 & 5235.5 & 5174.0 & 4.62 & -9999. & 4.56 & 0.85 & 0.57 \\
\hline
\end{tabular}

However, when we analyze the stars by class (main sequence, turnoff, subgiant, red giant), the scatter in the derived elemental abundance is drastically reduced to $0.03-0.04$ dex for most of the elements. As our sample covers a wide range in surface gravity, $1.78 \leqslant \log g \leqslant 4.71$, it is possible that the observed scatter is the signature of a physical process modifying the stellar atmospheric 
Table 3

Stellar Abundances

\begin{tabular}{|c|c|c|c|c|c|c|c|c|c|c|c|c|c|c|c|}
\hline 2Mass ID & {$[\mathrm{Fe} / \mathrm{H}]$} & {$[\mathrm{C} / \mathrm{H}]$} & {$[\mathrm{N} / \mathrm{H}]$} & {$[\mathrm{O} / \mathrm{H}]$} & {$[\mathrm{Na} / \mathrm{H}]$} & {$[\mathrm{Mg} / \mathrm{H}]$} & {$[\mathrm{Al} / \mathrm{H}]$} & {$[\mathrm{Si} / \mathrm{H}]$} & {$[\mathrm{K} / \mathrm{H}]$} & {$[\mathrm{Ca} / \mathrm{H}]$} & {$[\mathrm{Ti} / \mathrm{H}]$} & {$[\mathrm{V} / \mathrm{H}]$} & {$[\mathrm{Cr} / \mathrm{H}]$} & {$[\mathrm{Mn} / \mathrm{H}]$} & {$[\mathrm{Ni} / \mathrm{H}]$} \\
\hline \multicolumn{16}{|l|}{ Red Giant } \\
\hline 2M08492491+1144057 & 0.13 & -0.20 & 0.35 & 0.09 & 0.33 & 0.17 & 0.42 & 0.24 & 0.03 & 0.14 & 0.22 & 0.20 & 0.01 & 0.08 & 0.11 \\
\hline $2 \mathrm{M} 08503613+1143180$ & 0.02 & -0.07 & 0.14 & 0.04 & 0.08 & 0.02 & 0.18 & 0.13 & -0.04 & -0.01 & -0.07 & 0.19 & -0.01 & -0.03 & 0.02 \\
\hline 2M08504964+1135089 & 0.07 & -0.16 & 0.43 & 0.09 & 0.32 & 0.11 & 0.32 & 0.21 & 0.00 & 0.06 & 0.04 & 0.28 & -0.04 & 0.07 & 0.07 \\
\hline $2 \mathrm{M} 08511269+1152423$ & 0.07 & -0.16 & 0.29 & 0.11 & 0.32 & 0.13 & 0.47 & 0.26 & -0.01 & 0.10 & 0.12 & 0.17 & -0.04 & 0.08 & 0.03 \\
\hline 2M08511704+1150464 & 0.07 & -0.20 & 0.48 & 0.13 & 0.28 & 0.13 & 0.27 & 0.21 & -0.03 & 0.06 & 0.08 & -0.00 & 0.01 & 0.03 & 0.05 \\
\hline 2M08511897+1158110 & 0.06 & -0.20 & 0.38 & 0.13 & 0.14 & 0.07 & 0.26 & 0.19 & -0.04 & 0.05 & -0.02 & 0.09 & -0.01 & 0.05 & 0.06 \\
\hline 2M08512156+1146061 & 0.10 & -0.15 & 0.35 & 0.12 & 0.31 & 0.14 & 0.34 & 0.24 & 0.01 & 0.09 & 0.06 & 0.26 & 0.01 & 0.08 & 0.09 \\
\hline 2M08512618+1153520 & 0.06 & -0.19 & 0.33 & 0.10 & 0.37 & 0.11 & 0.41 & 0.25 & -0.05 & 0.06 & 0.08 & 0.14 & -0.08 & 0.05 & 0.02 \\
\hline 2M08512898+1150330 & 0.04 & -0.08 & 0.21 & 0.09 & 0.35 & 0.10 & 0.34 & 0.24 & -0.04 & 0.06 & 0.01 & 0.12 & -0.08 & 0.06 & 0.03 \\
\hline 2M08512990+1147168 & -0.05 & -0.14 & 0.39 & 0.05 & 0.35 & 0.07 & 0.31 & 0.11 & -0.15 & -0.03 & 0.02 & -0.02 & -0.06 & -0.07 & -0.07 \\
\hline 2M08513577+1153347 & 0.04 & -0.13 & 0.24 & 0.09 & 0.12 & 0.05 & 0.24 & 0.15 & -0.06 & 0.01 & 0.02 & 0.14 & -0.04 & 0.02 & 0.04 \\
\hline 2M08513938+1151456 & 0.06 & -0.18 & 0.30 & 0.12 & 0.22 & 0.08 & 0.36 & 0.19 & 0.00 & 0.05 & 0.07 & 0.21 & -0.05 & 0.03 & 0.03 \\
\hline 2M08514234+1150076 & 0.12 & -0.17 & 0.44 & 0.21 & 0.31 & 0.26 & 0.32 & 0.23 & -0.03 & 0.15 & 0.13 & -0.06 & 0.00 & 0.05 & 0.08 \\
\hline 2M08514388+1156425 & 0.07 & -0.18 & 0.30 & 0.11 & 0.29 & 0.13 & 0.41 & 0.27 & -0.02 & 0.07 & 0.06 & 0.14 & -0.04 & 0.07 & 0.02 \\
\hline $2 \mathrm{M} 08514507+1147459$ & 0.07 & -0.14 & 0.29 & 0.11 & 0.33 & 0.10 & 0.34 & 0.21 & -0.01 & 0.07 & 0.04 & 0.24 & -0.00 & 0.03 & 0.04 \\
\hline $2 \mathrm{M} 08514883+1156511$ & 0.04 & -0.15 & 0.18 & 0.14 & 0.19 & 0.04 & 0.25 & 0.18 & -0.10 & 0.03 & -0.04 & 0.14 & -0.10 & -0.02 & 0.04 \\
\hline 2M08515611+1150147 & 0.15 & -0.17 & 0.09 & 0.15 & 0.27 & 0.18 & 0.42 & 0.28 & 0.02 & 0.19 & 0.14 & 0.37 & 0.05 & 0.08 & 0.10 \\
\hline 2M08515952+1155049 & 0.04 & -0.17 & 0.31 & 0.07 & 0.26 & 0.08 & 0.39 & 0.23 & -0.06 & 0.04 & -0.01 & 0.16 & -0.05 & 0.02 & -0.00 \\
\hline 2M08521097+1131491 & 0.10 & -0.16 & 0.35 & 0.17 & 0.32 & 0.17 & 0.43 & 0.26 & 0.02 & 0.13 & 0.14 & 0.22 & 0.01 & 0.08 & 0.05 \\
\hline 2M08521656+1119380 & 0.03 & -0.19 & 0.27 & 0.12 & 0.37 & 0.14 & 0.43 & 0.22 & -0.03 & 0.09 & 0.09 & 0.04 & -0.07 & 0.04 & -0.02 \\
\hline $2 \mathrm{M} 08521856+1144263$ & 0.06 & -0.23 & 0.39 & 0.12 & 0.39 & 0.14 & 0.35 & 0.23 & -0.05 & 0.05 & 0.05 & 0.34 & -0.04 & 0.07 & 0.05 \\
\hline $2 \mathrm{M} 08522636+1141277$ & 0.05 & -0.19 & 0.33 & 0.20 & 0.17 & 0.10 & 0.25 & 0.20 & -0.04 & 0.04 & 0.10 & 0.14 & -0.04 & -0.04 & 0.06 \\
\hline $2 \mathrm{M} 08525625+1148539$ & 0.16 & -0.21 & 0.31 & 0.31 & 0.29 & 0.21 & 0.43 & 0.31 & 0.07 & 0.15 & 0.23 & 0.21 & 0.08 & 0.12 & 0.16 \\
\hline 2M08534672+1123307 & 0.11 & 0.03 & 0.11 & 0.05 & 0.31 & 0.13 & 0.38 & 0.29 & 0.05 & 0.09 & 0.10 & 0.28 & 0.04 & 0.04 & 0.12 \\
\hline 2M08493465+1151256 & -1.14 & -0.72 & 0.68 & -1.00 & -2.50 & -0.98 & -0.63 & -1.54 & -0.72 & -0.92 & -1.43 & -1.63 & -1.90 & -0.10 & -0.97 \\
\hline $2 \mathrm{M} 08505816+1152223$ & 0.08 & -0.05 & -0.01 & 0.03 & 0.26 & 0.09 & 0.28 & 0.20 & -0.02 & 0.11 & 0.05 & 0.18 & -0.01 & 0.05 & 0.09 \\
\hline 2M08510723+1153019 & -1.71 & -0.69 & -0.26 & -0.15 & -1.36 & -2.21 & -1.77 & -2.21 & -1.23 & -1.53 & -2.21 & -0.84 & -0.89 & -1.21 & -1.36 \\
\hline 2M08510839+1147121 & 0.07 & -0.15 & -0.08 & 0.26 & 0.16 & 0.09 & 0.27 & 0.21 & -0.01 & 0.06 & -0.03 & 0.30 & 0.01 & 0.04 & 0.06 \\
\hline $2 \mathrm{M} 08522003+1127362$ & 0.09 & -0.10 & -0.07 & 0.27 & 0.28 & 0.13 & 0.30 & 0.22 & -0.03 & 0.09 & 0.07 & 0.15 & -0.01 & 0.04 & 0.09 \\
\hline \multicolumn{16}{|l|}{ Subgiant } \\
\hline 2M08504994+1149127 & -0.05 & -0.02 & 0.20 & -0.05 & -0.07 & -0.03 & 0.04 & 0.05 & -0.09 & -0.06 & -0.10 & -0.04 & 0.01 & -0.11 & -0.04 \\
\hline $2 \mathrm{M} 08510325+1145473$ & 0.01 & 0.01 & -0.02 & 0.07 & -0.76 & -0.25 & -0.03 & -0.02 & -0.07 & 0.05 & -0.04 & -0.52 & -2.35 & -0.06 & -0.01 \\
\hline 2M08511564+1150561 & 0.04 & -0.11 & 0.10 & 0.15 & 0.16 & 0.02 & 0.19 & 0.11 & -0.00 & 0.00 & -0.00 & 0.14 & -0.07 & 0.02 & 0.07 \\
\hline $2 \mathrm{M} 08511670+1145293$ & 0.01 & -0.02 & -0.00 & 0.04 & 0.24 & -0.11 & 0.00 & 0.05 & -0.05 & 0.02 & 0.03 & -0.40 & 0.05 & -0.02 & -0.00 \\
\hline $2 \mathrm{M} 08512122+1145526$ & -0.05 & -0.02 & -0.05 & 0.02 & -0.55 & -0.24 & -0.06 & -0.11 & -0.07 & -0.06 & -0.26 & -0.36 & -2.34 & -0.10 & -0.01 \\
\hline 2M08512879+1151599 & 0.00 & -0.04 & -0.01 & -0.02 & 0.29 & -0.11 & 0.04 & 0.08 & -0.19 & -0.01 & 0.09 & -0.02 & -0.02 & -0.13 & -0.01 \\
\hline 2M08512935+1145275 & 0.01 & -0.14 & 0.16 & 0.08 & 0.24 & 0.00 & 0.12 & 0.12 & -0.06 & -0.01 & -0.11 & -0.14 & -0.03 & -0.02 & 0.01 \\
\hline 2M08513540+1157564 & 0.01 & -0.26 & 0.03 & 0.17 & 0.17 & -0.08 & 0.11 & 0.03 & -0.01 & -0.06 & -0.21 & 0.03 & -0.07 & -0.04 & 0.03 \\
\hline 2M08513862+1220141 & 0.02 & 0.03 & -0.03 & 0.09 & 0.17 & 0.01 & 0.13 & 0.08 & -0.07 & -0.04 & -0.12 & 0.21 & -0.03 & 0.01 & 0.06 \\
\hline 2M08514401+1146245 & 0.07 & 0.01 & 0.15 & -0.07 & 0.35 & 0.10 & 0.13 & 0.10 & -0.03 & -0.09 & 0.18 & -0.39 & 0.01 & 0.05 & 0.05 \\
\hline 2M08514474+1146460 & -0.01 & -0.17 & 0.14 & 0.11 & 0.19 & -0.03 & 0.08 & 0.03 & -0.09 & -0.05 & -0.12 & 0.09 & -0.09 & -0.02 & 0.03 \\
\hline 2M08514994+1149311 & -0.00 & 0.01 & -0.01 & 0.12 & -2.38 & 0.02 & 0.15 & 0.17 & 0.01 & 0.06 & 0.10 & 0.13 & -2.25 & -0.06 & 0.02 \\
\hline 2M08515335+1148208 & -0.06 & -0.06 & -0.07 & 0.05 & -0.95 & -0.04 & 0.10 & 0.07 & -0.01 & -0.01 & 0.06 & 0.04 & -2.17 & -0.12 & -0.02 \\
\hline 2M08521134+1145380 & 0.08 & -0.07 & 0.29 & 0.03 & 0.39 & 0.10 & 0.07 & 0.12 & -0.03 & 0.04 & 0.06 & -0.29 & -0.02 & 0.02 & 0.06 \\
\hline $2 \mathrm{M} 08503667+1148553$ & -0.07 & -0.04 & -0.06 & -0.09 & -1.34 & -0.09 & 0.12 & -0.02 & 0.05 & 0.01 & 0.03 & -0.03 & -2.38 & -0.20 & -0.07 \\
\hline $2 \mathrm{M} 08505569+1152146$ & 0.01 & 0.02 & -0.08 & 0.00 & 0.37 & 0.02 & 0.15 & 0.15 & -0.03 & 0.01 & -0.06 & 0.14 & -0.26 & -0.11 & 0.01 \\
\hline $2 \mathrm{M} 08510106+1150108$ & 0.08 & 0.04 & -0.08 & 0.34 & 0.15 & 0.08 & 0.16 & 0.18 & -0.03 & 0.09 & 0.17 & -0.04 & 0.08 & 0.04 & 0.06 \\
\hline
\end{tabular}


Table 3

(Continued)

\begin{tabular}{|c|c|c|c|c|c|c|c|c|c|c|c|c|c|c|c|}
\hline 2Mass ID & {$[\mathrm{Fe} / \mathrm{H}]$} & {$[\mathrm{C} / \mathrm{H}]$} & {$[\mathrm{N} / \mathrm{H}]$} & {$[\mathrm{O} / \mathrm{H}]$} & {$[\mathrm{Na} / \mathrm{H}]$} & {$[\mathrm{Mg} / \mathrm{H}]$} & {$[\mathrm{Al} / \mathrm{H}]$} & {$[\mathrm{Si} / \mathrm{H}]$} & {$[\mathrm{K} / \mathrm{H}]$} & {$[\mathrm{Ca} / \mathrm{H}]$} & {$[\mathrm{Ti} / \mathrm{H}]$} & {$[\mathrm{V} / \mathrm{H}]$} & {$[\mathrm{Cr} / \mathrm{H}]$} & {$[\mathrm{Mn} / \mathrm{H}]$} & {$[\mathrm{Ni} / \mathrm{H}]$} \\
\hline $2 \mathrm{M} 08510951+1141449$ & 0.15 & 0.07 & -0.04 & 0.25 & 0.23 & 0.13 & 0.23 & 0.27 & 0.01 & 0.16 & 0.22 & -0.15 & 0.18 & 0.15 & 0.15 \\
\hline $2 \mathrm{M} 08511877+1151186$ & 0.09 & -0.08 & -0.01 & -0.06 & 0.17 & 0.08 & 0.28 & 0.15 & 0.04 & 0.08 & 0.04 & 0.21 & -0.02 & 0.04 & 0.11 \\
\hline $\begin{array}{l}2 \mathrm{M} 08515567+1217573 \\
\text { Turnoff }\end{array}$ & & 0.14 & -2.20 & 0.03 & 0.20 & 0.14 & -0.10 & 0.05 & -0.04 & 0.09 & 0.01 & -0.01 & 0.06 \\
\hline $2 \mathrm{M} 08503392+1146272$ & 0.01 & 0.01 & $\cdots$ & $\cdots$ & $\cdots$ & -0.00 & 0.14 & 0.10 & -0.05 & -0.04 & -0.31 & 0.01 & $\cdots$ & -0.04 & 0.05 \\
\hline $2 \mathrm{M} 08504079+1147462$ & -0.05 & -0.03 & $\ldots$ & $\ldots$ & $\cdots$ & -0.04 & 0.07 & 0.13 & -0.09 & -0.03 & 0.22 & -0.31 & $\cdots$ & -0.18 & -0.02 \\
\hline $2 \mathrm{M} 08505177+1200247$ & -0.03 & -0.03 & $\cdots$ & $\cdots$ & $\cdots$ & -0.22 & -0.04 & -0.10 & -0.19 & -0.09 & -0.47 & 0.05 & $\cdots$ & -0.09 & -0.01 \\
\hline $2 \mathrm{M} 08505702+1159158$ & -0.08 & -0.07 & $\cdots$ & $\cdots$ & $\cdots$ & -0.26 & -0.03 & -0.13 & 0.03 & -0.03 & -0.06 & -0.10 & $\ldots$ & -0.14 & -0.02 \\
\hline $2 \mathrm{M} 08505762+1155147$ & -0.02 & -0.02 & $\cdots$ & $\cdots$ & $\cdots$ & -0.08 & 0.04 & 0.06 & -0.01 & -0.05 & -0.36 & -0.10 & $\ldots$ & -0.09 & -0.00 \\
\hline $2 \mathrm{M} 08505903+1148576$ & -0.03 & -0.02 & $\cdots$ & $\cdots$ & $\cdots$ & -0.26 & -0.10 & -0.02 & -0.03 & -0.07 & -0.53 & 0.03 & $\cdots$ & -0.11 & -0.05 \\
\hline 2M08505973+1139524 & -0.03 & -0.02 & $\cdots$ & $\cdots$ & $\cdots$ & -0.22 & -0.08 & 0.01 & -0.15 & -0.02 & -0.32 & -0.35 & $\cdots$ & -0.11 & 0.00 \\
\hline 2M08510969+1159096 & -0.11 & -0.12 & $\cdots$ & $\cdots$ & $\cdots$ & -0.34 & -0.15 & -0.15 & -0.10 & -0.09 & -0.20 & -0.28 & $\cdots$ & -0.19 & -0.12 \\
\hline 2M08511576+1152587 & -0.02 & -0.00 & $\cdots$ & $\cdots$ & $\cdots$ & -0.18 & 0.13 & -0.04 & 0.06 & 0.02 & 0.01 & -0.04 & $\cdots$ & -0.05 & 0.04 \\
\hline $2 \mathrm{M} 08512240+1151291$ & -0.12 & -0.11 & $\cdots$ & $\cdots$ & $\cdots$ & -0.28 & -0.18 & -0.17 & -0.06 & -0.16 & -0.22 & 0.03 & $\cdots$ & -0.16 & -0.05 \\
\hline 2M08513710+1154599 & -0.01 & -0.07 & $\cdots$ & $\cdots$ & $\cdots$ & -0.15 & -0.03 & 0.05 & -0.10 & 0.11 & -0.11 & -0.15 & $\cdots$ & -0.10 & 0.06 \\
\hline 2M08513806+1201243 & -0.01 & -0.04 & $\cdots$ & $\cdots$ & $\cdots$ & -0.25 & -0.10 & -0.06 & -0.02 & 0.11 & -0.28 & -0.05 & $\cdots$ & -0.12 & 0.04 \\
\hline $2 \mathrm{M} 08514122+1154290$ & -0.04 & -0.03 & $\cdots$ & $\cdots$ & $\cdots$ & -0.22 & -0.01 & -0.06 & -0.01 & -0.05 & -0.49 & 0.04 & $\cdots$ & -0.12 & -0.00 \\
\hline 2M08514475+1145012 & -0.10 & -0.08 & $\cdots$ & $\cdots$ & $\cdots$ & -0.32 & -0.19 & -0.11 & -0.04 & -0.14 & -0.14 & 0.26 & $\cdots$ & -0.13 & -0.06 \\
\hline $2 \mathrm{M} 08520741+1150221$ & -0.00 & -0.01 & $\cdots$ & $\cdots$ & $\cdots$ & -0.13 & 0.05 & 0.02 & -0.12 & 0.02 & -0.36 & -0.10 & $\cdots$ & -0.03 & 0.02 \\
\hline \multicolumn{16}{|l|}{ Main Sequence } \\
\hline 2M08502805+1154505 & 0.02 & -0.00 & $\cdots$ & $\ldots$ & $\ldots$ & -0.10 & 0.02 & -0.08 & -0.03 & 0.06 & 0.12 & -0.12 & $\cdots$ & -0.06 & -0.03 \\
\hline $2 \mathrm{M} 08511229+1154230$ & 0.04 & 0.01 & $\cdots$ & $\cdots$ & $\cdots$ & -0.08 & 0.01 & 0.03 & 0.06 & 0.06 & -0.00 & 0.06 & $\cdots$ & -0.04 & 0.05 \\
\hline 2M08512314+1154049 & 0.00 & -0.04 & $\cdots$ & $\cdots$ & $\cdots$ & -0.17 & -0.08 & -0.01 & -0.01 & 0.05 & -0.49 & 0.04 & $\cdots$ & -0.12 & 0.01 \\
\hline 2M08512604+1149555 & -0.02 & -0.13 & $\cdots$ & $\cdots$ & $\cdots$ & -0.05 & 0.15 & -0.08 & -0.01 & 0.03 & 0.03 & 0.02 & $\cdots$ & -0.12 & -0.02 \\
\hline 2M08512996+1151090 & -0.01 & -0.03 & $\cdots$ & $\cdots$ & $\cdots$ & -0.14 & -0.04 & -0.00 & 0.01 & 0.04 & -0.07 & 0.03 & $\cdots$ & -0.05 & 0.03 \\
\hline 2M08513119+1153179 & -0.03 & -0.03 & $\cdots$ & $\ldots$ & $\ldots$ & -0.19 & -0.10 & -0.04 & -0.07 & -0.06 & -0.19 & 0.04 & $\cdots$ & -0.07 & -0.01 \\
\hline $2 \mathrm{M} 08513701+1136516$ & 0.03 & 0.06 & $\ldots$ & $\cdots$ & $\cdots$ & 0.03 & 0.02 & 0.04 & -0.01 & 0.01 & 0.12 & 0.15 & $\cdots$ & 0.03 & 0.07 \\
\hline 2M08514189+1149376 & 0.06 & 0.02 & $\cdots$ & $\cdots$ & $\cdots$ & -0.10 & -0.09 & 0.12 & -0.05 & 0.14 & -0.11 & 0.07 & $\cdots$ & 0.02 & 0.11 \\
\hline 2M08514742+1147096 & 0.05 & -0.03 & $\cdots$ & $\cdots$ & $\cdots$ & 0.00 & 0.03 & 0.01 & -0.00 & 0.02 & 0.01 & 0.08 & $\cdots$ & 0.03 & 0.03 \\
\hline 2M08521649+1147382 & 0.02 & -0.04 & $\cdots$ & $\cdots$ & $\cdots$ & -0.15 & -0.06 & 0.07 & -0.03 & 0.05 & -0.33 & 0.13 & $\cdots$ & -0.01 & 0.07 \\
\hline 2M08505439+1156290 & -0.04 & -0.02 & $\cdots$ & $\cdots$ & $\cdots$ & -0.21 & -0.01 & -0.08 & -0.11 & -0.08 & -0.11 & -0.04 & $\cdots$ & -0.10 & 0.04 \\
\hline 2M08510076+1153115 & 0.02 & -0.02 & $\cdots$ & $\cdots$ & $\cdots$ & -0.13 & 0.10 & 0.04 & -0.02 & -0.03 & -0.08 & 0.11 & $\cdots$ & -0.05 & 0.05 \\
\hline 2M08511176+1150018 & 0.03 & -0.01 & $\cdots$ & $\cdots$ & $\cdots$ & -0.32 & -0.10 & 0.04 & -0.09 & 0.12 & -0.07 & 0.10 & $\cdots$ & -0.05 & 0.04 \\
\hline $2 \mathrm{M} 08512080+1145024$ & -0.03 & -0.03 & $\cdots$ & $\cdots$ & $\cdots$ & -0.21 & -0.12 & -0.02 & -0.24 & 0.03 & -0.20 & -0.84 & $\cdots$ & -0.13 & -0.04 \\
\hline 2M08512742+1153265 & -0.04 & -0.03 & $\cdots$ & $\cdots$ & $\cdots$ & -0.15 & 0.01 & -0.03 & -0.01 & -0.01 & -0.04 & -0.02 & $\cdots$ & -0.07 & 0.02 \\
\hline 2M08512788+1155409 & -0.03 & -0.02 & $\cdots$ & $\cdots$ & $\cdots$ & -0.20 & -0.01 & -0.11 & 0.02 & 0.01 & -0.09 & 0.04 & $\cdots$ & -0.07 & 0.09 \\
\hline 2M08513012+1143498 & -0.07 & -0.06 & $\cdots$ & $\cdots$ & $\cdots$ & -0.28 & -0.19 & -0.06 & -0.25 & -0.05 & -0.56 & -0.75 & $\cdots$ & -0.17 & -0.07 \\
\hline 2M08513455+1149068 & 0.06 & 0.02 & $\cdots$ & $\cdots$ & $\cdots$ & -0.12 & 0.01 & 0.09 & -0.01 & 0.13 & 0.05 & 0.06 & $\cdots$ & -0.01 & 0.09 \\
\hline 2M08521868+1143246 & -0.08 & -0.05 & $\cdots$ & $\cdots$ & $\cdots$ & -0.35 & -0.14 & -0.07 & -0.08 & -0.10 & -0.17 & 0.21 & $\cdots$ & -0.21 & -0.10 \\
\hline 2M08512643+1143506 & -0.21 & -0.67 & $\cdots$ & $\cdots$ & $\cdots$ & -0.16 & -1.56 & 0.26 & 0.13 & 0.58 & -0.66 & -2.11 & $\cdots$ & 0.54 & 0.04 \\
\hline $2 \mathrm{M} 08513259+1148520$ & -0.18 & -0.15 & $\cdots$ & $\cdots$ & $\cdots$ & -0.17 & -0.46 & 0.02 & -0.60 & -0.61 & -0.55 & -0.61 & $\cdots$ & -0.19 & -0.15 \\
\hline
\end{tabular}

Excluded sample due

to low $\mathrm{S} / \mathrm{N}(<100)$

Subgiant

\begin{tabular}{|c|c|c|c|c|c|c|c|c|c|c|c|c|c|c|c|}
\hline 2M08503438+1139566 & 0.10 & 0.07 & 0.06 & 0.09 & 0.72 & 0.03 & 0.24 & 0.25 & -0.05 & 0.20 & 0.16 & -0.08 & -0.25 & -0.08 & 0.08 \\
\hline $2 \mathrm{M} 08504198+1136525$ & 0.11 & 0.03 & 0.07 & 0.03 & 0.33 & 0.08 & 0.11 & 0.34 & 0.02 & 0.26 & 0.24 & -0.04 & 0.00 & -0.01 & 0.12 \\
\hline $2 \mathrm{M} 08510811+1201065$ & -0.07 & -0.13 & -0.12 & -0.32 & $\ldots$ & -0.13 & 0.36 & 0.03 & -0.09 & 0.18 & 0.29 & -0.26 & $\ldots$ & -0.18 & -0.05 \\
\hline
\end{tabular}


Table 3

(Continued)

\begin{tabular}{|c|c|c|c|c|c|c|c|c|c|c|c|c|c|c|c|}
\hline 2Mass ID & {$[\mathrm{Fe} / \mathrm{H}]$} & {$[\mathrm{C} / \mathrm{H}]$} & {$[\mathrm{N} / \mathrm{H}]$} & {$[\mathrm{O} / \mathrm{H}]$} & {$[\mathrm{Na} / \mathrm{H}]$} & {$[\mathrm{Mg} / \mathrm{H}]$} & {$[\mathrm{Al} / \mathrm{H}]$} & {$[\mathrm{Si} / \mathrm{H}]$} & {$[\mathrm{K} / \mathrm{H}]$} & {$[\mathrm{Ca} / \mathrm{H}]$} & {$[\mathrm{Ti} / \mathrm{H}]$} & {$[\mathrm{V} / \mathrm{H}]$} & {$[\mathrm{Cr} / \mathrm{H}]$} & {$[\mathrm{Mn} / \mathrm{H}]$} & {$[\mathrm{Ni} / \mathrm{H}]$} \\
\hline 2M08511826+1150196 & -0.17 & -0.21 & -0.20 & -0.39 & $\cdots$ & -0.29 & 0.07 & -0.12 & -0.15 & -0.03 & -0.12 & -0.24 & & -0.30 & -0.21 \\
\hline $2 \mathrm{M} 08520356+1141238$ & -0.04 & -0.02 & -0.05 & 0.01 & -0.56 & 0.04 & 0.08 & 0.11 & 0.05 & -0.01 & 0.20 & 0.09 & -0.22 & -0.27 & 0.01 \\
\hline \multicolumn{16}{|l|}{ Main Sequence } \\
\hline $2 \mathrm{M} 08502833+1142097$ & 0.01 & -0.02 & $\cdots$ & $\cdots$ & $\cdots$ & -0.20 & -0.05 & 0.16 & 0.05 & 0.05 & 0.27 & -0.05 & $\cdots$ & 0.05 & 0.09 \\
\hline 2M08503788+1252295 & 0.07 & 0.04 & $\cdots$ & $\cdots$ & $\cdots$ & -0.18 & -0.05 & 0.08 & -0.03 & 0.12 & -0.06 & 0.07 & $\cdots$ & -0.01 & 0.14 \\
\hline 2M08505334+1143399 & -0.10 & -0.13 & $\cdots$ & $\cdots$ & $\cdots$ & -0.60 & -0.11 & -0.07 & -0.36 & 0.04 & -0.41 & -0.50 & $\cdots$ & -0.18 & -0.05 \\
\hline 2M08505923+1146129 & -0.10 & -0.11 & $\cdots$ & $\cdots$ & $\cdots$ & -0.43 & -0.47 & -0.18 & -0.18 & -0.07 & -0.14 & -0.85 & $\cdots$ & -0.10 & -0.04 \\
\hline 2M08512386+1138521 & -0.04 & -0.07 & $\cdots$ & $\cdots$ & $\cdots$ & -0.25 & -0.14 & -0.09 & -0.10 & -0.10 & -0.47 & 0.07 & $\cdots$ & -0.10 & 0.02 \\
\hline 2M08513215+1136126 & -0.05 & -0.03 & $\cdots$ & $\cdots$ & $\cdots$ & -0.20 & -0.16 & -0.14 & -0.06 & -0.32 & 0.48 & -2.50 & $\cdots$ & 0.04 & 0.01 \\
\hline 2M08513444+1137574 & 0.02 & 0.02 & $\cdots$ & $\cdots$ & $\cdots$ & -0.28 & -0.10 & 0.01 & -0.24 & 0.05 & 0.35 & 0.75 & $\cdots$ & -0.04 & -0.02 \\
\hline $2 \mathrm{M} 08514375+1145148$ & -0.04 & -0.08 & $\cdots$ & $\cdots$ & $\cdots$ & -0.22 & -0.17 & -0.02 & 0.06 & -0.05 & 0.21 & 0.41 & $\cdots$ & 0.00 & 0.00 \\
\hline $2 \mathrm{M} 08514465+1141510$ & -0.02 & 0.02 & $\cdots$ & $\cdots$ & $\cdots$ & -0.19 & -0.19 & 0.03 & -0.07 & 0.03 & 0.17 & 0.43 & $\cdots$ & -0.16 & -0.00 \\
\hline 2M08515290+1146358 & 0.12 & 0.02 & $\cdots$ & $\cdots$ & $\cdots$ & -0.15 & -0.11 & 0.25 & -0.05 & 0.22 & 0.23 & 0.38 & $\cdots$ & 0.02 & 0.07 \\
\hline 2M08521664+1142300 & -0.15 & -0.10 & $\cdots$ & $\cdots$ & $\cdots$ & -0.31 & -0.16 & -0.13 & 0.22 & -0.16 & 0.04 & 0.29 & $\cdots$ & -0.29 & -0.09 \\
\hline $2 \mathrm{M} 08504511+1136023$ & 0.05 & -0.08 & $\cdots$ & $\cdots$ & $\cdots$ & -0.34 & -0.34 & 0.12 & 0.04 & 0.03 & -0.13 & -0.27 & $\cdots$ & -0.08 & -0.15 \\
\hline 2M08510131+1141587 & -0.17 & -0.19 & $\cdots$ & $\cdots$ & $\cdots$ & -0.42 & -0.23 & -0.13 & -0.39 & -0.11 & -0.43 & -0.36 & $\cdots$ & -0.23 & -0.10 \\
\hline 2M08510156+1147501 & -0.09 & -0.06 & $\cdots$ & $\cdots$ & $\cdots$ & -0.42 & -0.08 & -0.25 & -0.10 & -0.06 & -0.07 & -0.54 & $\cdots$ & -0.17 & -0.00 \\
\hline 2M08511229+1146212 & -0.05 & -0.02 & $\cdots$ & $\cdots$ & $\cdots$ & -0.29 & -0.08 & -0.12 & -0.14 & 0.05 & -0.03 & -0.40 & $\cdots$ & -0.04 & 0.01 \\
\hline 2M08511810+1142547 & -0.02 & 0.01 & $\cdots$ & $\cdots$ & $\cdots$ & -0.22 & -0.09 & -0.06 & -0.14 & -0.03 & -0.32 & -0.82 & $\cdots$ & -0.07 & -0.01 \\
\hline $2 \mathrm{M} 08512033+1145523$ & -0.03 & -0.01 & $\cdots$ & $\cdots$ & $\cdots$ & -0.21 & -0.06 & -0.01 & -0.22 & 0.01 & 0.03 & -0.32 & $\cdots$ & -0.05 & -0.03 \\
\hline 2M08512176+1144050 & -0.05 & -0.06 & $\cdots$ & $\cdots$ & $\cdots$ & -0.48 & -0.12 & -0.10 & -0.10 & 0.09 & -0.07 & -0.10 & $\cdots$ & -0.10 & -0.09 \\
\hline $2 \mathrm{M} 08512467+1143061$ & 0.03 & 0.01 & $\cdots$ & $\cdots$ & $\cdots$ & -0.47 & -0.05 & 0.13 & -0.34 & 0.11 & -0.38 & 0.21 & $\cdots$ & -0.16 & -0.07 \\
\hline 2M08513424+1145535 & 0.06 & 0.03 & $\cdots$ & $\cdots$ & $\cdots$ & -0.03 & 0.10 & 0.04 & -0.01 & 0.13 & 0.08 & 0.14 & $\cdots$ & 0.02 & 0.09 \\
\hline
\end{tabular}




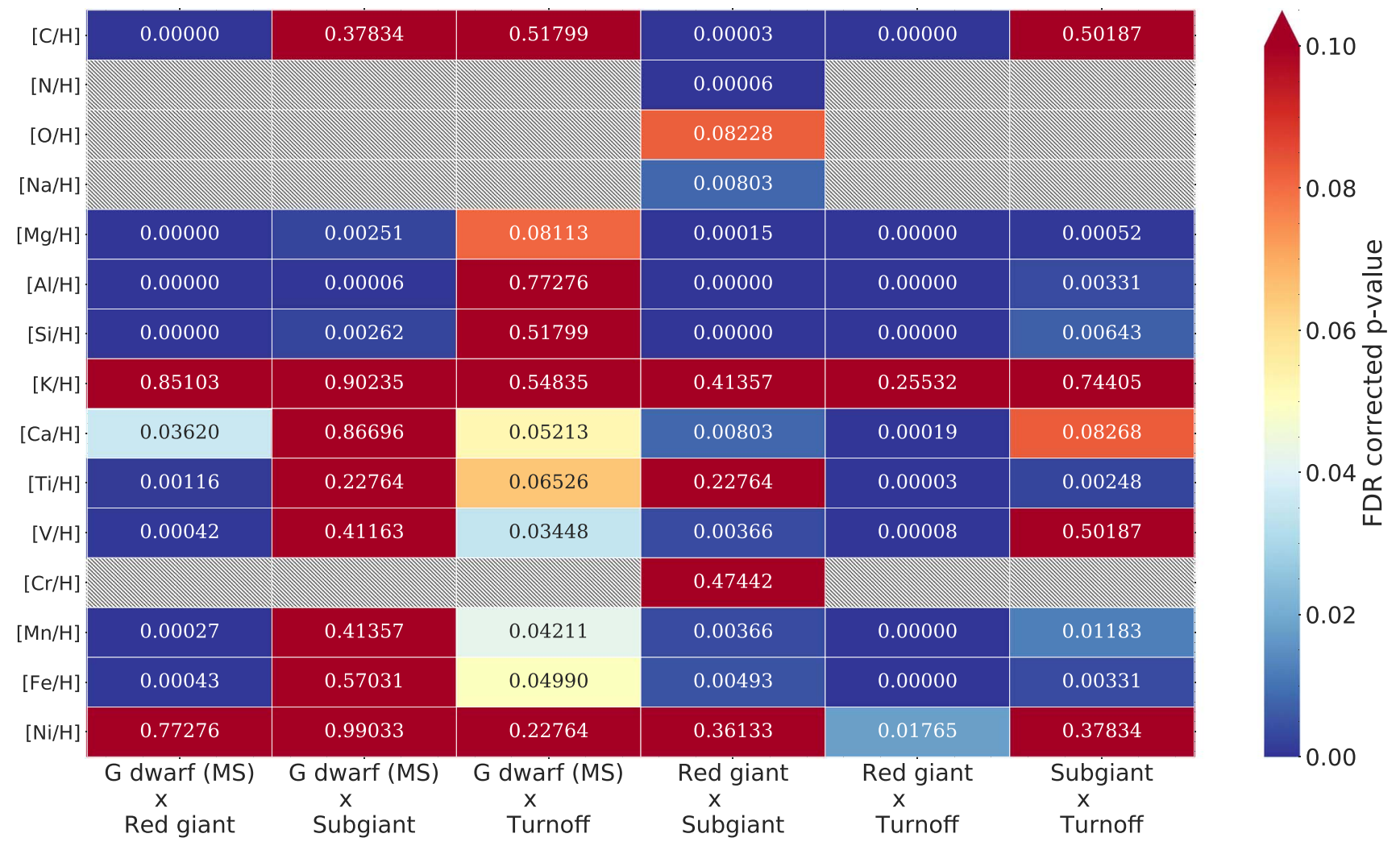

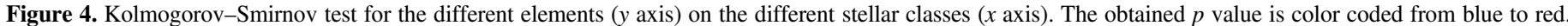
The red colors are saturated at 0.1 .

abundances, such as atomic diffusion as proposed by Souto et al. (2018). In the following sections, we discuss in detail the abundance trends as a function of the stellar parameters.

\subsection{Abundance Variations across the H-R Diagram in M67 Stars}

We split our sample into four different classes based on the stars' evolutionary stage. We selected as main-sequence stars those with $\log g \geqslant 4.20$, turnoff stars those with surface gravity $3.90<\log g<4.20$, subgiants those having $3.60 \leqslant \log g \leqslant$ 3.90 , and red giant stars those with $\log g<3.60$. (We note that the cut in surface gravity is similar to the one in color and magnitude, as can be seen in the right panel of Figure 1.)

Probing the level of homogeneity in open clusters is important to understanding their formation and for evaluating the possibility of performing chemical tagging in stellar populations. Chemical homogeneity in open clusters (as well as in globular clusters) is a critical assumption to understand changes in the abundances across evolutionary stages. Bovy (2016) and Price-Jones \& Bovy (2018), using APOGEE spectra, found tight constraints on the chemical homogeneity of M67 using a sample of red giant stars. Bovy (2016) analyzed 24 red giant stars in M67, finding one-dimensional sequences with a spread in the elemental initial cluster abundances lower than 0.03 dex ( $2 \sigma$ of uncertainty) for all elements studied in this work. It is worth noting that the Bovy (2016) results were derived in a way that is insensitive to the effects of atomic diffusion, mixing, and other physical processes that may modify the stellar surface abundances.
One straightforward way to evaluate if samples of stars have similar abundances is to apply a Kolmogorov-Smirnov test (K-S test). The K-S test is usually invoked to find out if two samples are drawn from the same distribution. We perform a study of chemical homogeneity of M67 stars using the derived abundances through a K-S test, and we apply it to the same classes, for example, red giants $\times$ red giants. To be able to compare the derived abundances for the same classes using the $\mathrm{K}-\mathrm{S}$ test, we randomly split each group into two samples and then we apply the K-S test. To ensure we do not choose a random split that favors homogeneity, for each group, we have run the test in 1000 random splits. This result shows that the abundances of each stellar class are indistinguishable, with the derived median $p$ value $>0.50$ for all elements in the four stellar classes. This is a complementary result to Bovy (2016), finding chemical homogeneity of M67 stars in the same evolutionary stage based on the stellar abundances derived in this work.

We also applied the K-S test using the derived abundances for the 15 studied elements comparing stars in the different groups: $\mathrm{G}$ dwarf main sequence $(\mathrm{MS}) \times$ red giant, $\mathrm{G}$ dwarf $(\mathrm{MS}) \times$ subgiant, $\mathrm{G}$ dwarf $(\mathrm{MS}) \times$ turnoff, red giant $\times$ subgiant, red giant $\times$ turnoff, and subgiant $\times$ turnoff stars.

In Figure 4 we present the results of the K-S two-sided test comparing the individual abundances for each stellar class. The vertical axis represents the $[\mathrm{X} / \mathrm{H}]$ derived here, and the horizontal axis represents the subgroups being compared. Each cell shows the $p$ value of the K-S test and is colored as shown in the side color bar. We designed the color scale to give a blue color if the samples are clearly distinct, a yellow color if the $p$ 


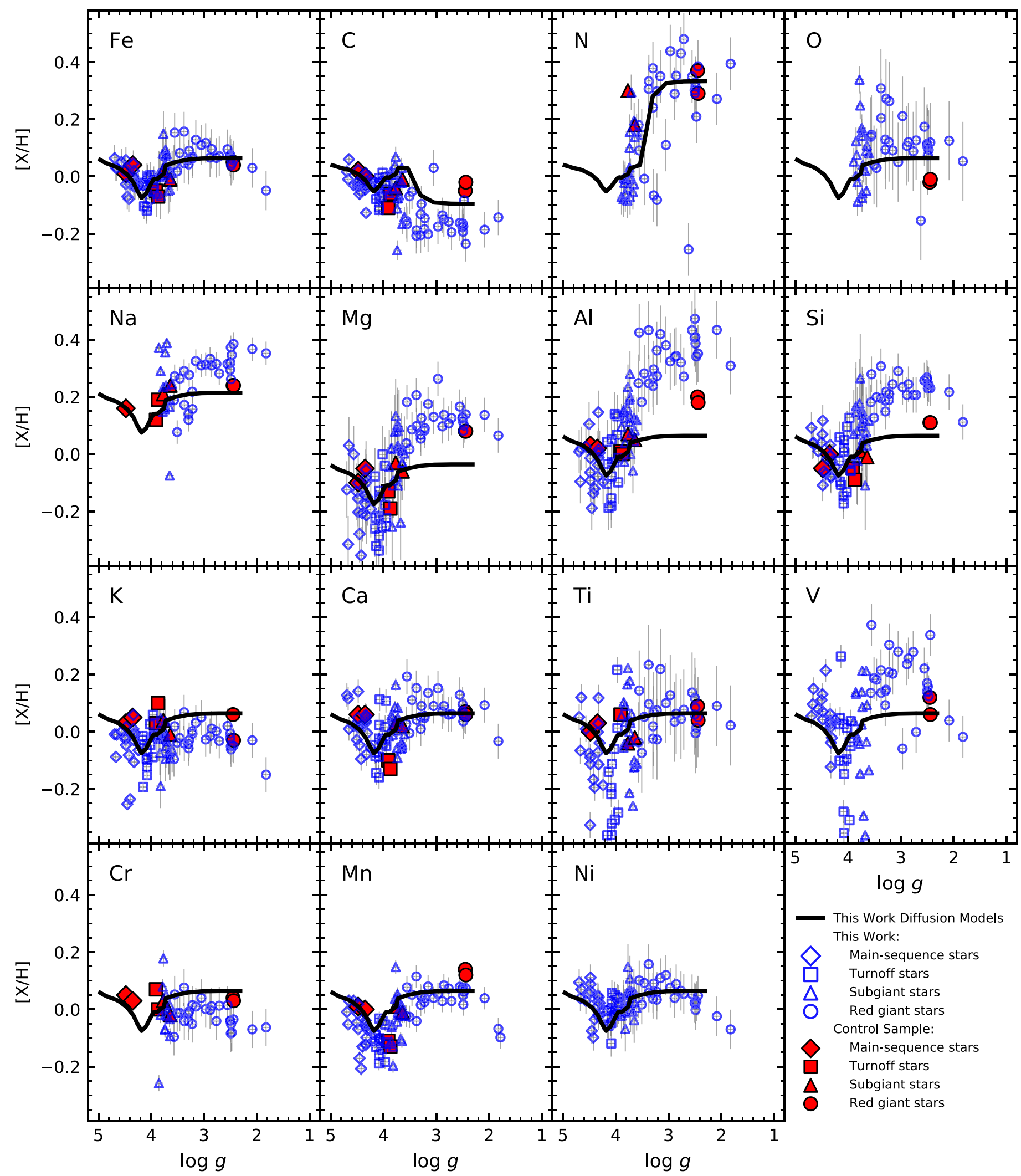

Figure 5. Chemical abundances for the studied stars are shown as a function of $\log g$. The symbol notation is similar to Figure 1 (open symbols instead of filled symbols). The diffusion models calculated in this work are shown as solid black lines.

value is near 0.05 , and a red color if we cannot reject the null hypothesis, that is, the samples are not distinguishable. Note that we have applied a false discovery rate (Benjamini \& Hochberg 1995) correction in order to account for the fact that we are performing many hypothesis tests simultaneously, and spurious rejections of the null hypothesis are therefore expected. Regardless of the threshold that we use, we obtain outstanding segregation for red giant and turnoff stars based on 


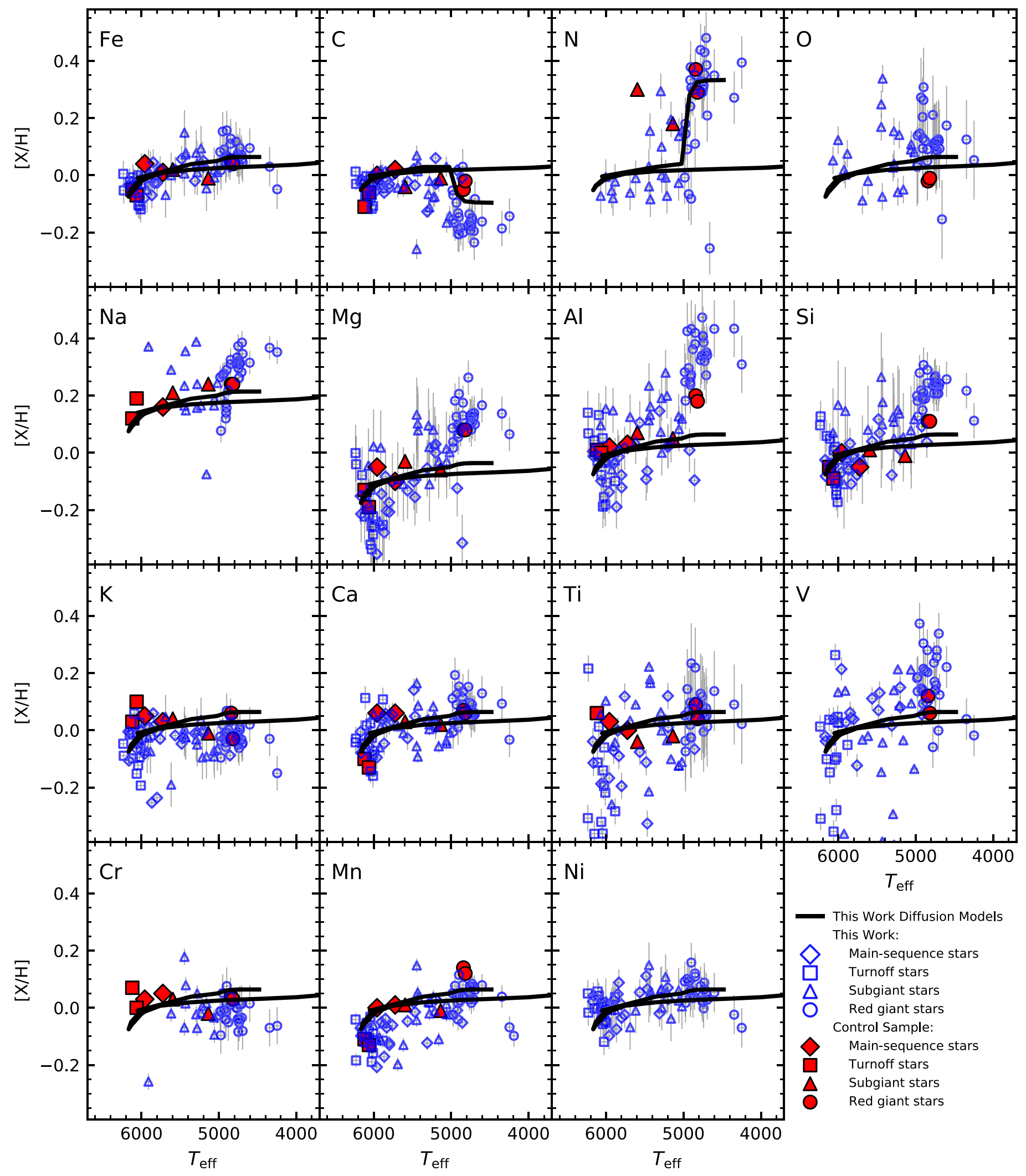

Figure 6. Same as Figure 5, except shown as a function of $T_{\text {eff }}$.

their abundances. The $\mathrm{K}$ abundance is the one with higher $p$ values $(>0.03)$ for all scenarios. On the other hand, the two classes most difficult to separate based on their abundances are the main-sequence and the turnoff stars. The abundances of
$\mathrm{Mg}, \mathrm{Ca}, \mathrm{V}$, and $\mathrm{Fe}$ are the best ones to distinguish between these classes. The $\mathrm{Mg}$ abundances show significant differences among all stellar classes (with $p$ values $<0.10$ for all comparisons). 


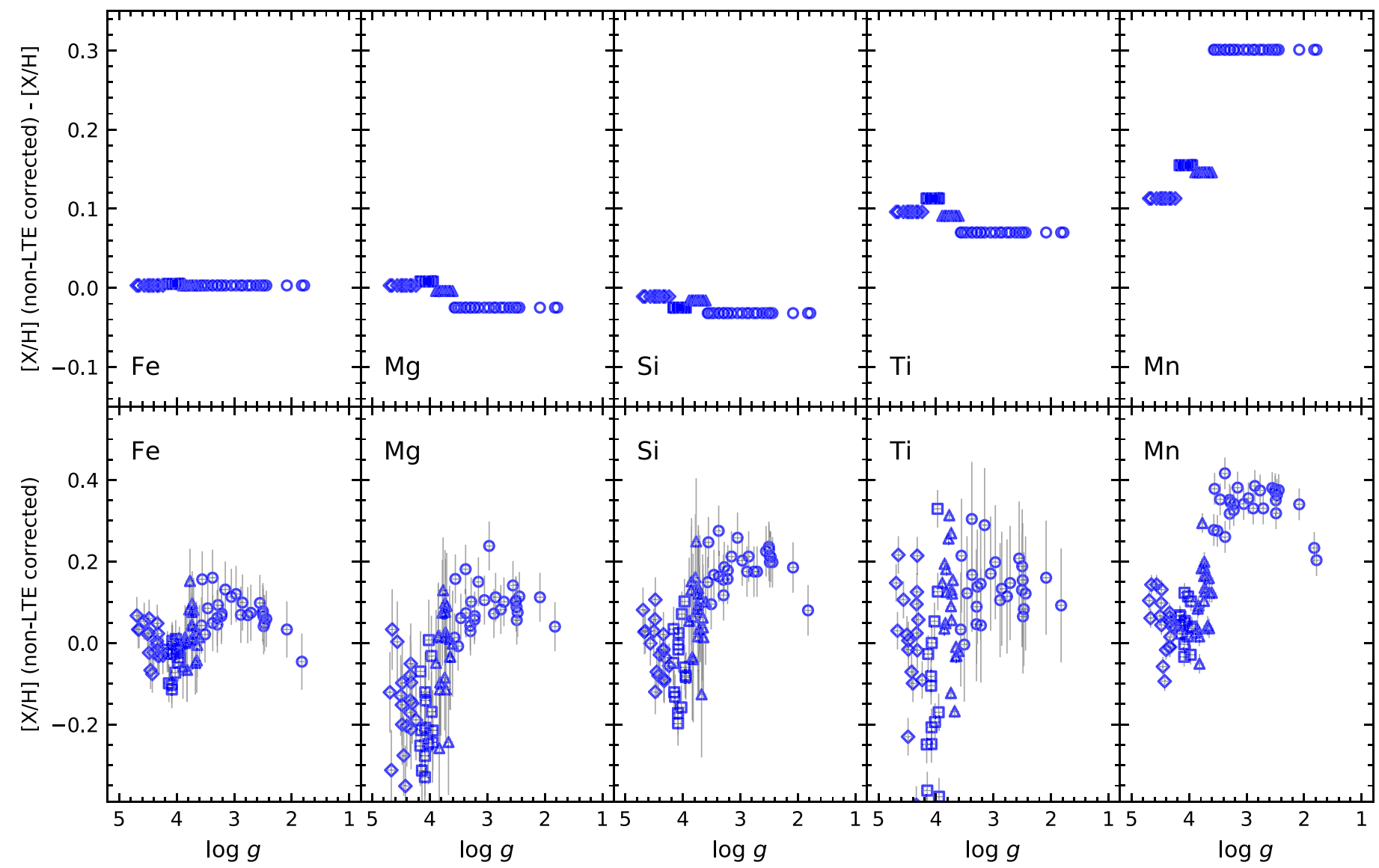

Figure 7. Top panel: $\log g$ vs. $[\mathrm{X} / \mathrm{H}]$ (corrected-derived) individual abundances from non-LTE deviation. Bottom panel: $\log g$ vs. $[\mathrm{X} / \mathrm{H}]$ corrected from non-LTE deviations. In both panels, the symbols follow the same notation as Figure 5.

\subsubsection{As a Function of Stellar Parameters}

In Figure 5 we display the derived individual abundances as a function of surface gravity for the 15 elements studied. We use the same symbol notation as in Figure 1, but with open symbols instead of filled. We also show the line-by-line manual abundance results from Souto et al. (2018), our control sample. Atomic diffusion models computed for this work (see Section 6) are overplotted for each element (C and $\mathrm{N}$ including mixing processes). We note that the diffusion models for $\mathrm{Na}$ and $\mathrm{Mg}$ abundances were slightly shifted in order to better fit the observed abundances.

From visual inspection-and in agreement with the results from the K-S test-we can organize the element variations as a function of surface gravity (as well as $T_{\text {eff }}$ and $M_{\star}$ ) into three groups of elements: (1) $\mathrm{C}$ and $\mathrm{N}$, with abundances displaying a different behavior for the evolved subgiant and red giant stars (as a consequence of dredge-up mechanisms); (2) $\mathrm{O}, \mathrm{Na}$, and $\mathrm{Cr}$ as their abundances are not reliable for the main-sequence and turnoff stars because their spectral lines become too weak; (3) the elements showing a dip, either sinuous or small, in the elemental abundance close to $\log g=4.00 \operatorname{dex}(\mathrm{Fe}, \mathrm{Mg}, \mathrm{Al}, \mathrm{Si}$, $\mathrm{K}, \mathrm{Ca}, \mathrm{Ti}, \mathrm{V}, \mathrm{Mn}$, and $\mathrm{Ni}$ ). The derived abundances of $\mathrm{Mg}, \mathrm{Al}$, and Si present the most significant changes between the stellar classes (excluding $\mathrm{N}$ ), where the red giant abundances are 0.10 to 0.20 dex higher than those from the subgiants.

In Figure 6, we present the abundance results as a function of $T_{\text {eff }}$ in M67 stars, with diffusion models also shown. Overall, the behavior seen in Figure 6 indicates an abundance increase (in the range $0.00-0.40 \mathrm{dex}$ ) as $T_{\text {eff }}$ decreases from 6000 to
$4000 \mathrm{~K}$. The elements showing a smooth increase or decrease in abundance as functions of $T_{\text {eff }}$ are $\mathrm{Fe}, \mathrm{Ca}$, and $\mathrm{Mn}$. The elements most sensitive to $T_{\text {eff }}, \mathrm{Na}, \mathrm{Mg}, \mathrm{Al}$, and $\mathrm{Si}$, show a monotonic increase in their individual abundances. Similar to the trends with $\log g, \mathrm{C}$ shows a particular behavior, and the abundance variation of $\mathrm{N}$ shows a maximum value around $T_{\text {eff }} \sim 4700 \mathrm{~K}$ and then decreases for higher and lower values of $T_{\text {eff }}$. The elements presenting the least sensitivity to $T_{\text {eff }}$ are $\mathrm{K}, \mathrm{Cr}$, and $\mathrm{Ni}$. Ti and $\mathrm{V}$ show the most significant abundance scatter in the analysis as a function of both $\log g$ and $T_{\text {eff. }}$

Souto et al. (2018) showed that atomic diffusion processes can explain the abundance variations of M67 stars across the different evolutionary stages. However, other physical processes are also relevant in the context of abundance variations, where the most significant sources of deviations, not precisely in order, are non-LTE effects, 1D or 3D treatment of the model atmosphere, stellar rotation $(v \sin i)$, mixing process (e.g., first dredge-up), and atomic diffusion processes. In the following sections, we discuss the impact of these possible deviations in our results.

\section{Possible Explanations for the Abundance Trends}

Figures 5 and 6 show significant abundance variations as a function of the stellar parameters ( $\log g$ and $\left.T_{\text {eff }}\right)$. Such abundance trends are not expected to occur in open clusters - due to the homogeneity of the stars formed by the same material-unless some additional effect or mechanism is playing a role in the stellar atmosphere, or in the abundance determination itself. 


\subsection{Non-LTE Deviations in the Near Infrared}

Deviations from the LTE have been studied mostly at optical wavelengths, where strong deviations are found to occur in metal-poor, evolved red giant stars (Asplund 2005; Asplund et al. 2009). In the near infrared (NIR), in particular in the $H$ band, the works of Cunha et al. (2015) and Zhang et al. $(2016,2017)$ have investigated non-LTE effects in Na I, Mg I, and $\mathrm{Si}$ I lines in the APOGEE spectra, finding deviations from non-LTE in these elements to be usually smaller than 0.05 dex (see also the discussion in Souto et al. 2018). Using the results from Bergemann \& Gehren (2008) and Bergemann et al. (2012a, 2013, 2015) compiled from a Maria Bergemann website (nlte.mpia.de), we created a grid of non-LTE deviations for five elements: $\mathrm{Fe}, \mathrm{Mg}, \mathrm{Si}, \mathrm{Ti}$, and $\mathrm{Mn}$. The deviations were estimated for each stellar class, assuming a solar metallicity and $T_{\text {eff }}=4700 \mathrm{~K}, \log g=2.40$, and $\xi=1.60 \mathrm{~km} \mathrm{~s}^{-1}$ for red giants; $T_{\text {eff }}=5400 \mathrm{~K}, \log g=3.70$, and $\xi=1.25 \mathrm{~km} \mathrm{~s}^{-1}$ for subgiants; $T_{\text {eff }}=6100 \mathrm{~K}, \log g=3.90$, and $\xi=1.15 \mathrm{~km} \mathrm{~s}^{-1}$ for turnoff stars; and $T_{\text {eff }}=5850 \mathrm{~K}, \quad \log g=4.40$, and $\xi=1.00 \mathrm{~km} \mathrm{~s}^{-1}$ for main-sequence stars. We adopted 1D plane-parallel models computed with MAFAGS-OS for all stellar classes. In Table 4 we summarize the average non-LTE correction for each stellar class and element.

In Figure 7 we show the non-LTE corrected abundances for the five elements studied ( $\mathrm{Fe}, \mathrm{Mg}, \mathrm{Si}, \mathrm{Ti}$, and $\mathrm{Mn}$ ). The top panel displays the abundance differences from $[\mathrm{X} / \mathrm{H}]_{\text {non-LTE }}-[\mathrm{X} / \mathrm{H}]_{\mathrm{LTE}}$, and in the bottom panel we show a plot similar to Figure 5, but now using the $[\mathrm{X} / \mathrm{H}]_{\text {non-LTE }}$.

The iron abundances do not show significant non-LTE deviations, as seen in Table 4, where $\delta$ (non-LTE-LTE) are smaller than 0.01 dex for all stellar classes. For $\mathrm{Mg}$ and $\mathrm{Si}$, the deviation is very similar for main-sequence stars, both positive, being almost null for $\mathrm{Mg}$. For subgiant and red giant stars, we obtain small, negative non-LTE corrections. The deviations for $\mathrm{Ti}$ and $\mathrm{Mn}$ are more significant in this study. For $\mathrm{Ti}$, the deviations are positive for the stellar classes studied here, with the major deviation observed in turnoff stars $(\delta$ (non-LTE$\mathrm{LTE})=0.11 \mathrm{dex}$ ). When applying non-LTE corrections, we do not see a strong change in the abundance versus $\log g$ diagram, when compared to the LTE one presented in Figure 5. The abundances of $\mathrm{Ti}$ are shifted in all classes, resulting in a higher scatter as a function of $\log g$. The Mn corrections show the most significant differences, $\delta$ (non-LTE-LTE) $\sim 0.13$ dex for main sequence, turnoff, and subgiants, and $\delta$ (non-LTELTE) $\sim 0.30$ dex for red giants. The inclusion of non-LTE corrections in the analysis does not erase the observed abundance trends in the different stellar classes.

\section{2. $1 D$ or 3D Model Atmospheres}

Stellar atmospheres are 3D and time-dependent; however, by convenience, we usually treat model atmospheres as having $1 \mathrm{D}$ plane-parallel or spherical geometry in hydrostatic equilibrium. This approximation simplifies the analysis, but can lead to systematic errors in the derived quantities (atmospheric parameters or chemical abundances).

The use of a 1D treatment of the stellar atmosphere requires the inclusion of "ad hoc" parameters to account for velocities that broaden the profiles at microscopic (microturbulence) and macroscopic (macroturbulence) levels. A precise determination of the microturbulence parameter minimizes the deviations from the results obtained with 3D models.
Table 4

Non-LTE Corrections

\begin{tabular}{lccccc}
\hline \hline Stellar Class & $\mathrm{Mg}$ & $\mathrm{Si}$ & $\mathrm{Ti}$ & $\mathrm{Mn}$ & $\mathrm{Fe}$ \\
\hline Main sequence & +0.003 & -0.011 & +0.096 & +0.113 & +0.003 \\
Turnoff & +0.008 & -0.025 & +0.113 & +0.155 & +0.005 \\
Subgiant & -0.004 & -0.016 & +0.091 & +0.146 & +0.003 \\
Red giant & -0.025 & -0.032 & +0.070 & +0.301 & +0.003 \\
\hline
\end{tabular}

As in non-LTE studies, 3D effects are also transition dependent, and analyses for NIR $H$-band transitions have been limited. The studies of Asplund (2005), Asplund et al. (2009), and Caffau et al. (2011) have summarized various effects and corrections for elemental abundances using optical spectra as a reference. In this section, we will summarize these effects for solar-metallicity stars to verify whether the abundance trends discussed in Section 4 could be explained by 3D effects.

Caffau et al. (2011) determined solar abundances from a 3D non-LTE analysis using the $\mathrm{CO}^{5} \mathrm{BOLD}$ code, providing 3D abundance corrections for several elements. For Fe, Caffau et al. (2011) find 3D corrections to be about 0.03 dex using the solar spectrum. The $\mathrm{C}$ abundance reported by Caffau et al. (2011) has a -0.02 dex 3D correction, while for $\mathrm{K}$ the authors obtain a correction of 0.07 dex. From Caffau et al. (2009), the solar $\mathrm{N}$ abundance is reported to have a $3 \mathrm{D}$ correction smaller than 0.01 dex.

The previous work by Bergemann et al. (2012b) studied the 3D deviations for stars in different evolutionary stages at different metallicities. They find 3D effects in the iron abundance for the Sun to be very small: 3D corrections $\sim 0.01$ dex. More recently, Bergemann et al. (2017) studied the $\mathrm{Mg}$ abundances in the Sun and found 3D corrections to be $\sim 0.02$ dex. Amarsi \& Asplund (2017) studied non-LTE 3D Si abundances in the Sun and found corrections to be lower than 0.01 dex. Amarsi et al. (2016), analyzing the O I forbidden line at $630 \mathrm{~nm}$, find $3 \mathrm{D}$ corrections to the $\mathrm{O}$ abundance to be between 0.05 and 0.20 dex, negative in the Sun and reaching higher values for turnoff stars.

All 3D corrections discussed above are smaller than 0.05 dex (except for $\mathrm{K}$ ), which is at the limit of the measurement uncertainties of this work. Given the small 3D corrections found for main-sequence stars, as well as the lack of studies in the literature for turnoff, subgiants, and red giant stars at solar metallicity, we conclude that deviations from 3D modeling are not enough to explain the abundance trends observed in this work.

\subsection{Stellar Rotation}

The study of the relation between stellar rotation and abundance variations in late-type stars is often motivated by the investigation of lithium depletion. Several authors have found correlations between stellar rotation and the lithium abundance depletion, such as Balachandran (1990, 1995), King et al. (2000), da Silva et al. (2009), Canto Martins et al. (2011), and Delgado Mena et al. (2014). None of the spectra analyzed in this study exhibit measurable rotational broadening $(v \sin (i))$ above the limits set by the APOGEE spectral resolution of $\sim 7-8 \mathrm{~km} \mathrm{~s}^{-1}$. 


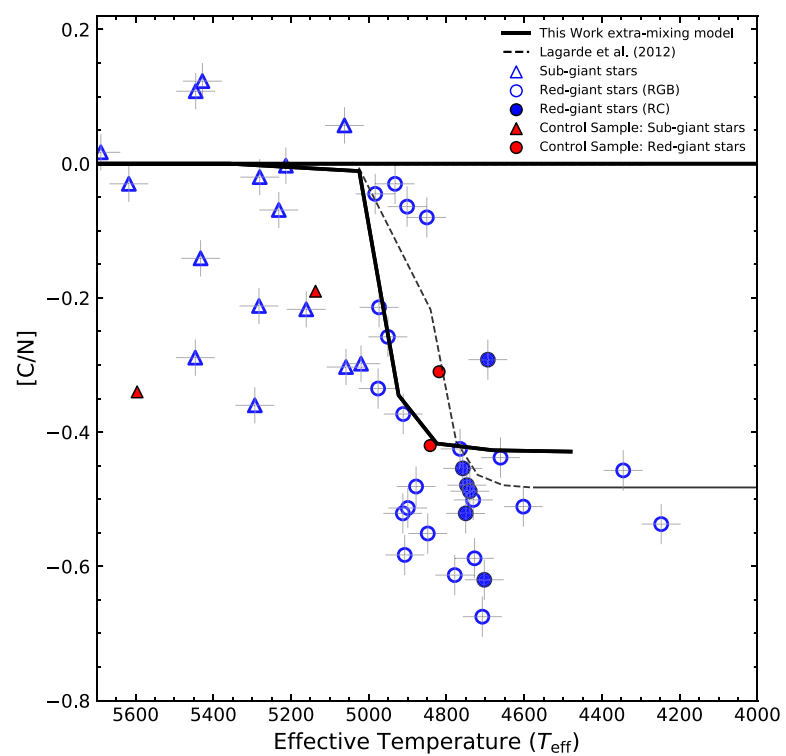

(a)

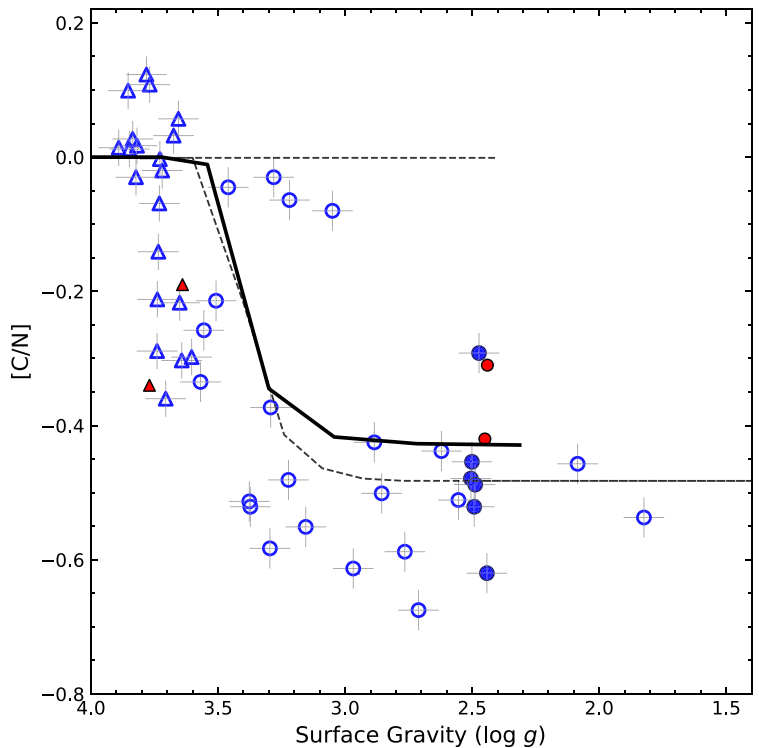

(b)

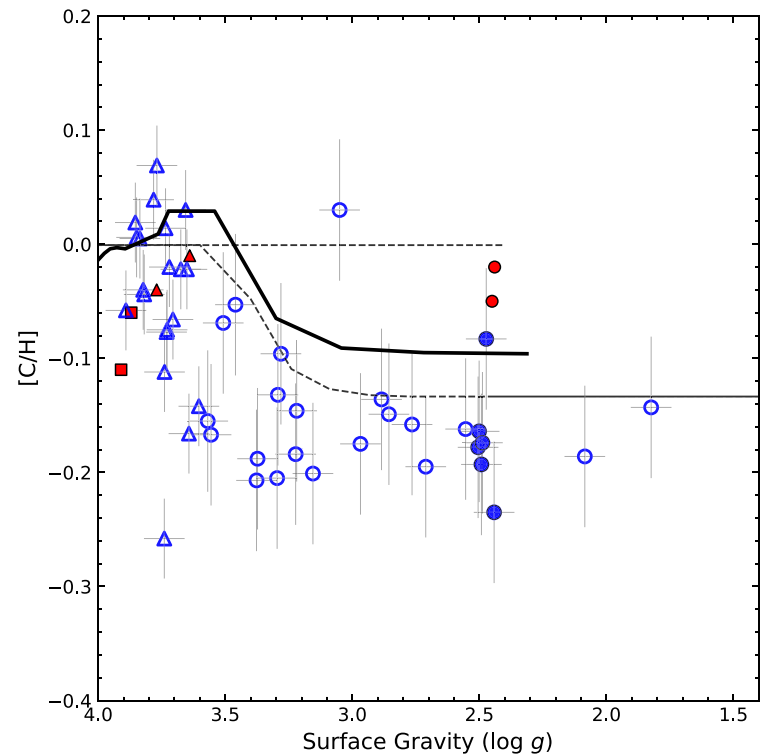

(c)

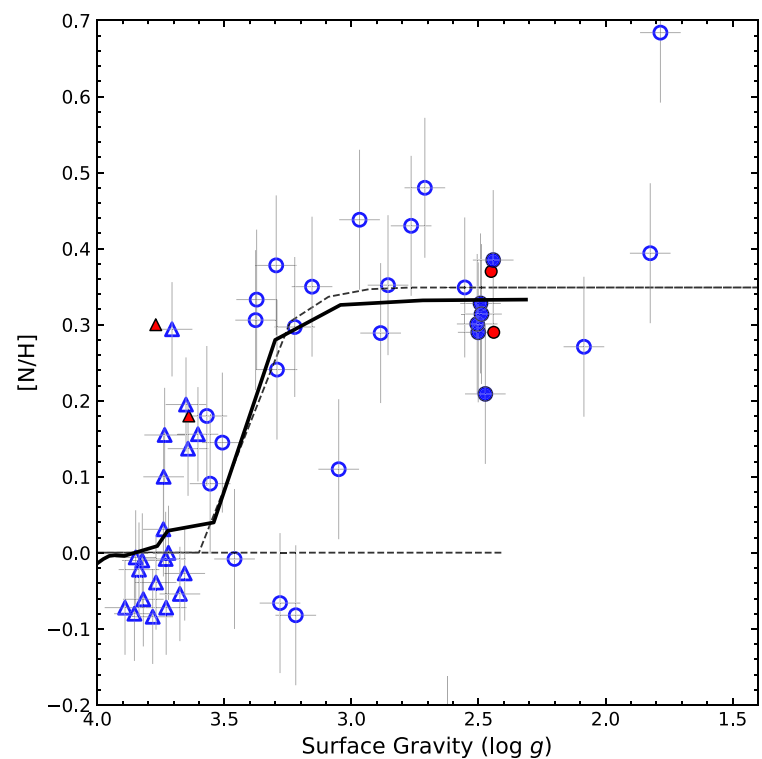

(d)

Figure 8. Panels (a) and (b) show $T_{\text {eff }}$ vs. $[\mathrm{C} / \mathrm{N}]$ and $\log g$ vs. $[\mathrm{C} / \mathrm{N}]$, respectively. Panels (c) and (d) present the $[\mathrm{C} / \mathrm{H}]-\log g$ and $[\mathrm{N} / \mathrm{H}]-\log g$ diagrams. We display the mixing models from this work (solid black curve) and from Lagarde et al. (2012, dashed black curve) as a comparison. All symbols follow the same notation as Figure 5, with the inclusion of filled circles for the red clump stars.

\section{Discussion}

The abundance results obtained for M67 stars show evidence that both mixing and atomic diffusion are operating, so stellar evolution models that include diffusion will be compared to the observationally derived abundances.

\subsection{Stellar Evolution Models}

We computed our mixing and atomic diffusion models using solar models (solar metallicity and solar age $4 \mathrm{Gyr}$ ) to calibrate the degree of gravitational settling precisely (using the surface solar $\mathrm{He}$ as a proxy); this gives a predicted reduction in the efficiency of the settling of $15 \%$, or an effective coefficient of 0.85 . The methodology adopted in the modeling of mixing and atomic diffusion is described in detail in Bahcall et al. (2001) and Delahaye \& Pinsonneault (2006). We note that, overall, our models agree with the ones from MIST (Choi et al. 2016,
Dotter et al. 2017); however, our models cover all of the species studied in this work, while the MIST models are not available for $\mathrm{Al}, \mathrm{K}, \mathrm{V}, \mathrm{Cr}, \mathrm{Mn}$, and $\mathrm{Ni}$.

\subsection{Mixing Processes: First Dredge-up}

When a low-mass star, such as a $\sim 1.2 M_{\odot}$ M67 star that is currently evolving off of the main sequence and across the subgiant branch, reaches the base of the RGB, the outer convective envelope reaches its largest extent in mass. At this point in the H-R diagram (where $T_{\text {eff }} \sim 5000 \mathrm{~K}$ and $\log g \sim 3.5$ in M67), the base of the convective envelope ingests material that has been exposed previously to $\mathrm{H}$ burning via the $\mathrm{CN}$ cycle. As a consequence of $\mathrm{CN}$-cycle $\mathrm{H}$ burning, this nuclearprocessed material contains an enhanced abundance of ${ }^{14} \mathrm{~N}$ and a decreased abundance of ${ }^{12} \mathrm{C}$. The convective envelope will carry this mixture to the surface, resulting in a lower surface 
abundance of ${ }^{12} \mathrm{C}$ and a larger abundance of ${ }^{14} \mathrm{~N}$ for stars evolving onto the RGB; this phase of stellar evolution is referred to as first dredge-up, or FDU (Iben 1965; for a more recent overview of the various red giant dredge-up episodes, see Karakas \& Lattanzio 2014). In the case of dredge-up in M67 red giants, the ${ }^{14} \mathrm{~N}$ abundance is predicted to be enhanced by roughly $\sim+0.30$ to $+0.40 \mathrm{dex}$, while the ${ }^{12} \mathrm{C}$ abundance is predicted to be depleted by $\sim-0.10$ to -0.20 dex. The magnitudes of the abundance changes in $\mathrm{C}$ and $\mathrm{N}$ are a function of red giant mass (Iben 1965), with larger-mass stars having deeper convective envelopes that dredge up more nuclear-processed material, resulting in larger ${ }^{14} \mathrm{~N}$ enhancements and larger ${ }^{12} \mathrm{C}$ depletions, producing lower $\mathrm{C} / \mathrm{N}$ ratios.

The expected relationship between red giant mass and $\mathrm{C} / \mathrm{N}$ ratio has been exploited by a number of recent studies using APOGEE data and results (e.g., Martig et al. 2016; Ness et al. 2016; see also D. Feuillet et al. 2019, in preparation) to produce age-mass relations as a function of red giant $[\mathrm{C} / \mathrm{N}]$ abundances, while Masseron \& Gilmore (2015) have analyzed $[\mathrm{C} / \mathrm{N}]$ to study the possible formation of the thin and thick disk.

In addition to standard convection in $1 \mathrm{D}$, other physical processes can modify the interior abundance profiles in stars as they evolve from the main sequence, across the subgiant branch, and onto the RGB, with two important processes being rotation and the inversion of the mean molecular weight gradient in a small region outside the H-burning shell created by ${ }^{3} \mathrm{He}$ burning via ${ }^{3} \mathrm{He}\left({ }^{3} \mathrm{He}, 2 \mathrm{p}\right) \alpha$ (Eggleton et al. 2006; Charbonnel \& Zahn 2007); this last process is referred to as thermohaline mixing. The inclusion of rotation-induced mixing and thermohaline mixing produces larger carbon depletions and larger nitrogen enhancements as a result of FDU. In this section, we use ${ }^{12} \mathrm{C}$ and ${ }^{14} \mathrm{~N}$ abundances derived here to compare with predictions from various models of first dredgeup mixing.

As shown in Figure 8, the M67 red giants display clear evidence of the first dredge-up through the behavior of the $\mathrm{C}$ and $\mathrm{N}$ abundances as functions of both $T_{\text {eff }}$ and $\log g$ (which map the position of a star along the subgiant and RGB); observed APOGEE abundances are plotted as the various symbols, while models are plotted as the continuous lines and are models from this study, along with those from Lagarde et al. (2012). The left panels of Figure 8 plot the $[\mathrm{C} / \mathrm{N}]$ values versus $T_{\text {eff }}$ (top, (a)) and $\log g$ (bottom, (b)), with the $[\mathrm{C} / \mathrm{N}]$ values decreasing rapidly at $T_{\text {eff }} \sim 5000 \mathrm{~K}$ and $\log g \sim 3.5$, right at the base of the RGB as predicted by FDU. The right panels plot the individual abundances of ${ }^{12} \mathrm{C}$ (as $\left.[\mathrm{C} / \mathrm{H}]\right)$ and ${ }^{14} \mathrm{~N}$ (as $[\mathrm{N} / \mathrm{H}]$ ) versus $\log g$. Carbon and nitrogen abundance differences between red giants on the RGB relative to those in the RC were found to agree with results from Tautvaišiene et al. (2000) and Masseron et al. (2017), who found slightly lower values of $\mathrm{C} / \mathrm{N}$ in $\mathrm{RC}$ stars compared to those on the RGB. Our values for M67 stars are $\left\langle{ }^{12} \mathrm{C} /{ }^{14} \mathrm{~N}\right\rangle_{\mathrm{RGB}}=1.86$ and \langle $\left.{ }^{12} \mathrm{C} /{ }^{14} \mathrm{~N}\right\rangle_{\mathrm{RC}}=1.40$, excluding the two evolved stars with $\log g<2.1$ dex, which places them on the upper RGB or possibly in an early-AGB phase of evolution.

Figure 8 also highlights differences in the $\mathrm{C}$ and $\mathrm{N}$ abundance variations predicted from mixing models when compared to those abundances derived in this study. In the left panels of Figure 8 (a) and (b), we show the $[\mathrm{C} / \mathrm{N}]$ ratio as a function of $T_{\text {eff }}$ and $\log g$, respectively, and we note that the overall observational results follow the model predictions, although the observed $[\mathrm{C} / \mathrm{N}]$ values are systematically lower. Such a difference can be a consequence of an overestimated nitrogen abundance in our analysis (as pointed out by Bertelli Motta et al. 2017 using ASPCAP data), due to a subestimated $\log g$. In the right panels of Figure 8 , we present the $[\mathrm{C} / \mathrm{H}]$ (panel (c)) and $[\mathrm{N} / \mathrm{H}]$ (panel (d)) abundances as a function of $\log g$. For nitrogen, the abundances are in agreement with the models; however, the observational carbon abundances differ from the models by $\sim-0.15$ dex. We conclude that the abundance variations observed for ${ }^{12} \mathrm{C}$ and ${ }^{14} \mathrm{~N}$ in the subgiant and red giant stars can be explained well by FDU mixing models. The mixing models here (as well as from Lagarde et al. 2012) predict changes for the other elemental abundances to be smaller than 0.01 dex as the star evolves. Therefore, mixing models cannot explain their abundance variations.

\subsection{Atomic Diffusion}

Atomic diffusion is a likely explanation for most of the observed abundance variations across the H-R diagram in M67, thus adding members of this old open cluster to those stars in which diffusion has been observed. Evidence of diffusion in the Sun is found both in its surface helium abundance, which is lower than the initial value, and in the solar sound speed profile being best fit by models that include diffusion (Bahcall et al. 1995; Chaboyer et al. 1995a). Lithium abundances settle at a rate similar to $\mathrm{He}$, and the flatness of the Spite Li plateau is likely set by diffusion (Chaboyer et al. 1992). The diffusion signature can be altered or erased by mixing, for example, mixing driven by rotation and dredge-up (see Section 6.2), thus complicating the detection and interpretation of diffusion patterns. Such mixing processes are likely at work in the Sun, which has a smoother composition profile than that predicted by diffusion alone, with the magnitude of diffusion being overestimated by about $25 \%$. This is also confirmed by looking at A-type stars: if they rotate fast enough, they are not chemically peculiar (Michaud 1970, see also Michaud et al. 2015). The interplay between diffusion creating abundance signatures that various mixing processes can then modify means that there are not necessarily firm theoretical predictions about the amplitude of the diffusion signature and its mass or metallicity dependence. Reasonably well-motivated trends can be expected, though, and Chaboyer et al. (1995b, 1995c), Choi et al. (2016), Dotter (2016), and Dotter et al. (2017) have approximated limits on how efficient diffusion can be in thin surface convective zones.

A few previous studies have probed atomic diffusion in cluster stars, with most of them focused on low-metallicity globular clusters: Korn et al. (2007), Lind et al. (2008), and Nordlander et al. (2012). The latter analyzed stars belonging to the globular cluster NGC 6397, with a metallicity $[\mathrm{Fe} / \mathrm{H}]=-2.00$ and age $13.5 \mathrm{Gyr}$, with their sample containing stars from the turnoff point (TOP) up to the RGB. The abundances of $\mathrm{Li}, \mathrm{Mg}, \mathrm{Ca}, \mathrm{Ti}, \mathrm{Cr}$, and $\mathrm{Fe}$ in those studies were derived from high-resolution optical spectroscopy, and they found that changes in the stellar abundances for different evolutionary phases are in good agreement with predictions from diffusion models from the literature; see Richard et al. (2002, 2005). In particular, Nordlander et al. (2012) found abundance differences of 0.06 and 0.18 dex between TOP and RGB stars in NGC 6397, with the largest difference for $\mathrm{Mg}$, and which is a much smaller variation than we see in M67, for 


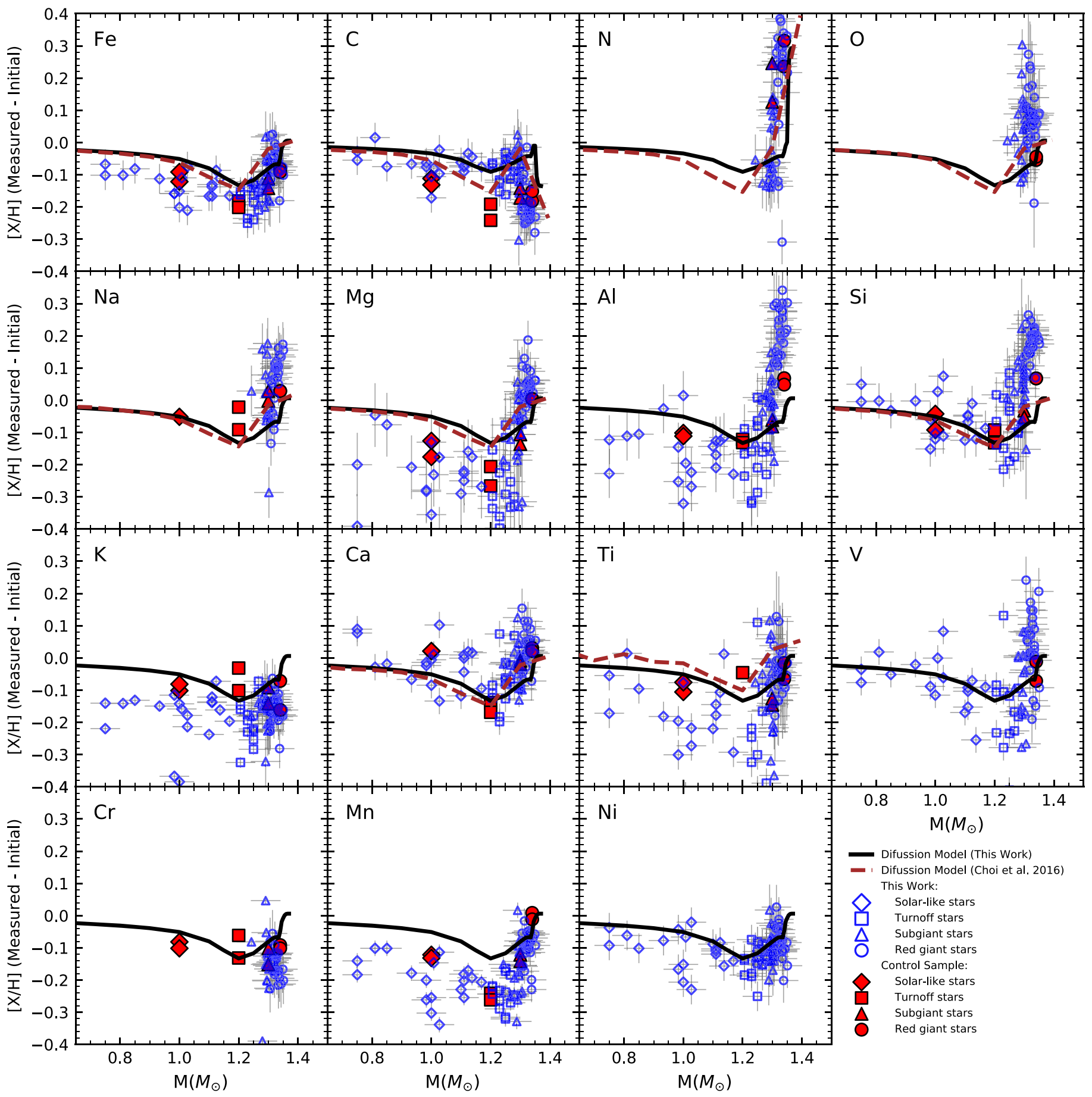

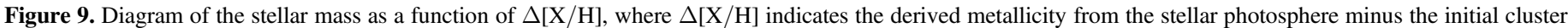

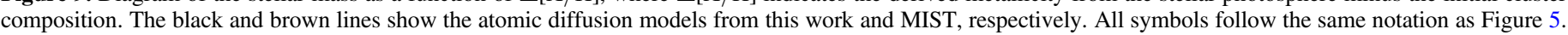

example for $\mathrm{Mg}$ or $\mathrm{Al}$. Of course, the chemical abundance of NGC 6397 is rather distinct from that of M67.

Önehag et al. (2014) found some evidence of atomic diffusion operating in M67. This was later supported by the abundance results in Blanco-Cuaresma et al. (2015). As previously mentioned, abundance differences of up to $\sim 0.20$ dex between the turnoff and red giant stars were observed by Souto et al. (2018) for the elements O, Na, Mg, $\mathrm{Al}, \mathrm{Si}, \mathrm{Ca}, \mathrm{V}, \mathrm{Mn}$, and Fe. A comparison of the results in Souto et al. (2018) with the diffusion patterns from the models by Choi et al. (2016) and Dotter et al. (2017) indicated good agreement. In addition, Bertelli Motta et al. (2018) found $>0.15$ dex abundance differences from main-sequence to red giant stars for the elements $\mathrm{Al}, \mathrm{Si}, \mathrm{Mn}$, and Ni. Gao et al. (2018) found a good match between the abundance variations for $\mathrm{Al}$ and $\mathrm{Si}$ with diffusion models.

\subsection{Atomic Diffusion in M67 Stars}

We find significant abundance differences (up to $\sim 0.50 \mathrm{dex}$ ) for most of the studied species between main-sequence, turnoff, subgiant, and red giant stars in M67. Using the K-S test 
(Figure 4), we obtained clear evidence of abundance differences between stars in different evolutionary stages in M67. In addition, we showed (Section 4) that the abundances of stars belonging to the same evolutionary class are indistinguishable.

In Figure 9 we present the mass $-\Delta[\mathrm{X} / \mathrm{H}]$ $\left([\mathrm{X} / \mathrm{H}]_{\text {Current }}-[\mathrm{X} / \mathrm{H}]_{\text {Initial }}\right)$ diagram for the 12 studied elements. Similar diagrams with abundances as a function of surface gravity and effective temperature are presented in Figures 5 and 6 .

In all three figures, we show the atomic diffusion models computed in this work as solid black lines and the MIST models as brown dashed lines. The pristine Fe abundance in the models is assumed to be the mean $\mathrm{Fe}$ abundance for the red giants, which is used as the fiducial point (i.e., $\delta[\mathrm{Fe} / \mathrm{H}]=0.00$ ) for the initial cluster value. We note that all other abundance ratios are assumed to be solar, that is, $[\mathrm{X} / \mathrm{Fe}]=0.00$. The abundance variations across the H-R diagram indicate that atomic diffusion is operating in most of the studied elements. The models for all of the elements display similar trends driven by atomic diffusion, except for $\mathrm{C}$ and $\mathrm{N}$, which include mixing signatures.

The complex trend observed in the carbon abundances is a consequence of diffusion operating in the main-sequence and turnoff stars (smaller convective envelopes), and mixing at the first dredge-up being responsible for the carbon abundance variation in subgiant and red giant stars (Section 6.2; Figure 8). These results suggest that atomic diffusion dominates over mixing in the main-sequence and turnoff stars, while mixing processes control the abundance changes in subgiant and red giant stars.

The nitrogen abundance variation can be explained as a signature of first dredge-up (Section 6.2). For oxygen, the scattered abundance results for red giant and subgiant stars, combined with the lack of results for main-sequence and turnoff stars, impedes detection of signatures of diffusion. Due to the weakness of $\mathrm{CN}$ and $\mathrm{OH}$ molecular lines in the APOGEE spectra of main-sequence and turnoff stars, it is not possible to derive $\mathrm{N}$ and $\mathrm{O}$ abundances in such stars.

The comparison of the abundance patterns for all elements with the model predictions indicates an overall good match between the atomic diffusion models and the derived abundances across the H-R diagram. However, the derived abundances exhibit a more significant dip across the mainsequence-turnoff stars when compared to what is expected from the atomic diffusion models, in particular for $\mathrm{Mg}, \mathrm{Al}, \mathrm{Ti}$, and $\mathrm{Mn}$.

For $\mathrm{Al}, \mathrm{Mg}, \mathrm{Si}$, and to a lesser degree $\mathrm{V}$, the relative dip across the main-sequence-turnoff stars is more significant because the red giant abundances are higher than those predicted by the models. (The $\mathrm{Na}$ abundances of red giants are also higher than the models, but there are no abundances for turnoff and main-sequence stars.) On the one hand, for $\mathrm{Ti}$ and $\mathrm{Mn}$, the dip is more considerable because the abundances of turnoff and main-sequence stars are lower. As discussed in Section 5 (Figure 7), non-LTE corrections for $\mathrm{Mg}$ and $\mathrm{Si}$ (as well as $\mathrm{Fe}$ ) would reduce the abundance dip by a factor of $\sim 0.03$ dex, while for $\mathrm{Ti}$, the dip would be reduced by roughly 0.05 dex. The non-LTE corrections for Mn, on the other hand, would systematically change the red giant abundances and increase the abundance difference between turnoff and red giant stars by $\sim 0.14$ dex, which would worsen the comparison with the models.

\section{Summary}

Given its combination of age and metallicity in addition to the numerous detailed studies in the literature, M67 remains a prime cluster to test for physical and chemical processes in stellar spectroscopy that are not yet well understood.

In this paper, we present individual abundances of 15 elements ( $\mathrm{C}, \mathrm{N}, \mathrm{O}, \mathrm{Na}, \mathrm{Mg}, \mathrm{Al}, \mathrm{Si}, \mathrm{K}, \mathrm{Ca}, \mathrm{Ti}, \mathrm{V}, \mathrm{Cr}, \mathrm{Mn}, \mathrm{Fe}$, and Ni) derived from a 1D LTE analysis of 83 stars in M67. The abundances were obtained via $\chi^{2}$ minimization of the high-resolution SDSS-IV/APOGEE spectra with the qASPCAP code. The stellar sample is composed of stars in different evolutionary stages (19 main sequence, 15 turnoff, 20 subgiant, and 29 red giants) with the aim to quantify abundance trends across the different stellar evolutionary phases (Önehag et al. 2014; Bertelli Motta et al. 2018; Gao et al. 2018; Souto et al. 2018).

We obtain significant abundance differences (of up to $0.30-0.40$ dex $)$ as a function of stellar parameters $\left(T_{\text {eff }}, \log g\right.$, and mass) that map the different stellar evolutionary classes. Studying the abundance variations within the same stellar classes, we find a much lower scatter, of about $\sim 0.05-0.10$ dex. Using the K-S test, it is found that the abundances within each stellar class are indistinguishable; while performing the test for the different classes, we obtain clear segregations for the red giant and turnoff stars in most of the elements analyzed.

We compiled non-LTE corrections for $\mathrm{Fe}, \mathrm{Mg}, \mathrm{Al}, \mathrm{Si}$, and Mn, finding them to be small for all elements $(<0.10 \mathrm{dex})$, except for $\mathrm{Mn}$, which had corrections between 0.15 and 0.30 dex in all stellar classes analyzed. With the use of nonLTE corrections to our derived abundances, we still observe clear abundance trends across the H-R diagram.

We found that mixing models explain well the abundance variations of $\mathrm{C}$ and $\mathrm{N}$ for subgiants and red giant stars. We see atomic diffusion operating in the $\mathrm{C}$ abundances in stars from the main-sequence-TOP. The atomic diffusion models computed in this work (as well as from the literature) predict reasonably well the remaining abundance patterns for the stars at different evolutionary stages studied in this work, and therefore we conclude that atomic diffusion operates in M67 more efficiently in the turnoff stars and in most of the elements (C, Mg, Al, Si, K, Ca, Ti, V, Mn, Fe, and Ni) analyzed in this work.

We thank the anonymous referee for useful comments that helped improve the paper. D.S. thanks Pascal Petit for the cordial hosting at CNRS-Toulouse, where part of this project was developed. K.C. and V.S. acknowledge that their work here is supported, in part, by the National Aeronautics and Space Administration under Grant 16-XRP16_2-0004, issued through the Astrophysics Division of the Science Mission Directorate. D.A.G.H. and O.Z. acknowledge support from the State Research Agency (AEI) of the Spanish Ministry of Science, Innovation and Universities (MCIU) and the European Regional Development Fund (FEDER) under grant AYA201788254-P. H.J. acknowledges support from the Crafoord Foundation, Stiftelsen Olle Engkvist Byggmästare, and Ruth och Nils-Erik Stenbäcks stiftelse.

Funding for the Sloan Digital Sky Survey IV has been provided by the Alfred P. Sloan Foundation, the U.S. Department of Energy Office of Science, and the Participating Institutions. SDSS-IV acknowledges support and resources 
from the Center for High-Performance Computing at the University of Utah. The SDSS website is www.sdss.org.

SDSS-IV is managed by the Astrophysical Research consortium for the Participating Institutions of the SDSS Collaboration including the Brazilian Participation Group, the Carnegie Institution for Science, Carnegie Mellon University, the Chilean Participation Group, the French Participation Group, Harvard-Smithsonian Center for Astrophysics, Instituto de Astrofísica de Canarias, The Johns Hopkins University, Kavli Institute for the Physics and Mathematics of the Universe (IPMU)/University of Tokyo, Lawrence Berkeley National Laboratory, Leibniz Institut für Astrophysik Potsdam (AIP), Max-Planck-Institut für Astronomie (MPIA Heidelberg), MaxPlanck-Institut für Astrophysik (MPA Garching), Max-PlanckInstitut für Extraterrestrische Physik (MPE), National Astronomical Observatory of China, New Mexico State University, New York University, University of Notre Dame, Observatório Nacional/MCTI, The Ohio State University, Pennsylvania State University, Shanghai Astronomical Observatory, United Kingdom Participation Group, Universidad Nacional Autónoma de México, University of Arizona, University of Colorado Boulder, University of Oxford, University of Portsmouth, University of Utah, University of Virginia, University of Washington, University of Wisconsin, Vanderbilt University, and Yale University.

\section{ORCID iDs}

Diogo Souto (i) https://orcid.org/0000-0002-7883-5425 C. Allende Prieto (i) https://orcid.org/0000-0002-0084-572X Marc Pinsonneault (1) https://orcid.org/0000-0002-7549-7766 Jo Bovy (ib https://orcid.org/0000-0001-6855-442X Jon Holtzman (i) https://orcid.org/0000-0002-9771-9622 J. A. Johnson (1) https://orcid.org/0000-0001-7258-1834 Henrik Jönsson (i) https://orcid.org/0000-0002-4912-8609 Steve R. Majewski (1) https://orcid.org/0000-0003-2025-3147 Matthew Shetrone (ํ) https://orcid.org/0000-0003-0509-2656 Kaike Pan iㅜ https://orcid.org/0000-0002-2835-2556

\section{References}

Abolfathi, B., Aguado, D. S., Aguilar, G., et al. 2018, ApJS, 235, 42 Allende Prieto, C., Beers, T. C., Wilhelm, R., et al. 2006, ApJ, 636, 804 Aller, L. H., \& Chapman, S. 1960, ApJ, 132, 461

Alvarez, R., \& Plez, B. 1998, A\&A, 330, 1109

Amarsi, A. M., \& Asplund, M. 2017, MNRAS, 464, 264

Amarsi, A. M., Asplund, M., Collet, R., \& Leenaarts, J. 2016, MNRAS, 455, 3735

Asplund, M. 2005, ARA\&A, 43, 481

Asplund, M., Grevesse, N., Sauval, A. J., \& Scott, P. 2009, ARA\&A, 47, 481

Bahcall, J. N., Pinsonneault, M. H., \& Basu, S. 2001, ApJ, 555, 990

Bahcall, J. N., Pinsonneault, M. H., \& Wasserburg, G. J. 1995, RvMP, 67, 781

Bailer-Jones, C. A. L., Rybizki, J., Fouesneau, M., Mantelet, G., \& Andrae, R. 2018, AJ, 156, 58

Balachandran, S. 1990, ApJ, 354, 310

Balachandran, S. 1995, ApJ, 446, 203

Bellini, A., Bedin, L. R., Pichardo, B., et al. 2010, A\&A, 513, A51

Benjamini, Y., \& Hochberg, Y. 1995, Journal of the Royal Statistical Society, 57, 289

Bergemann, M., Collet, R., Amarsi, A. M., et al. 2017, ApJ, 847, 15

Bergemann, M., \& Gehren, T. 2008, A\&A, 492, 823

Bergemann, M., Kudritzki, R.-P., Gazak, Z., Davies, B., \& Plez, B. 2015, ApJ, 804, 113

Bergemann, M., Kudritzki, R.-P., Plez, B., et al. 2012a, ApJ, 751, 156

Bergemann, M., Kudritzki, R.-P., Würl, M., et al. 2013, ApJ, 764, 115

Bergemann, M., Lind, K., Collet, R., Magic, Z., \& Asplund, M. 2012b, MNRAS, 427, 27
Bertelli Motta, C., Pasquali, A., Richer, J., et al. 2018, MNRAS, 478, 425 Bertelli Motta, C., Salaris, M., Pasquali, A., \& Grebel, E. K. 2017, MNRAS, 466, 2161

Bertran de Lis, S., Allende Prieto, C., Majewski, S. R., et al. 2016, A\&A, 590, A74

Blanco-Cuaresma, S., Soubiran, C., Heiter, U., et al. 2015, A\&A, 577, A47

Blanton, M. R., Bershady, M. A., Abolfathi, B., et al. 2017, AJ, 154, 28

Bovy, J. 2016, ApJ, 817, 49

Bressan, A., Marigo, P., Girardi, L., et al. 2012, MNRAS, 427, 127

Caffau, E., Ludwig, H.-G., Steffen, M., Freytag, B., \& Bonifacio, P. 2011, SoPh, 268, 255

Caffau, E., Maiorca, E., Bonifacio, P., et al. 2009, A\&A, 498, 877

Canto Martins, B. L., Lèbre, A., Palacios, A., et al. 2011, A\&A, 527, A94

Castelli, F., \& Kurucz, R. L. 2004, arXiv:astro-ph/0405087

Chaboyer, B., Deliyannis, C. P., Demarque, P., Pinsonneault, M. H., \& Sarajedini, A. 1992, ApJ, 388, 372

Chaboyer, B., Demarque, P., Guenther, D. B., \& Pinsonneault, M. H. 1995a, ApJ, 446, 435

Chaboyer, B., Demarque, P., \& Pinsonneault, M. H. 1995b, ApJ, 441, 865

Chaboyer, B., Demarque, P., \& Pinsonneault, M. H. 1995c, ApJ, 441, 876

Chapman, S. 1917a, MNRAS, 77, 540

Chapman, S. 1917b, MNRAS, 77, 539

Charbonnel, C., \& Zahn, J.-P. 2007, A\&A, 467, L15

Choi, J., Dotter, A., Conroy, C., et al. 2016, ApJ, 823, 102

Cohen, J. G. 1980, ApJ, 241, 981

Cunha, K., Smith, V. V., Hasselquist, S., et al. 2017, ApJ, 844, 145

Cunha, K., Smith, V. V., Johnson, J. A., et al. 2015, ApJL, 798, L41

da Silva, L., Torres, C. A. O., de La Reza, R., et al. 2009, A\&A, 508, 833

Delahaye, F., \& Pinsonneault, M. H. 2006, ApJ, 649, 529

Delgado Mena, E., Israelian, G., González Hernández, J. I., et al. 2014, A\&A, 562, A92

De Silva, G. M., Freeman, K. C., Asplund, M., et al. 2007, AJ, 133, 1161

De Silva, G. M., Freeman, K. C., Bland-Hawthorn, J., et al. 2015, MNRAS, 449, 2604

De Silva, G. M., Sneden, C., Paulson, D. B., et al. 2006, AJ, 131, 455

Dotter, A. 2016, ApJS, 222, 8

Dotter, A., Conroy, C., Cargile, P., \& Asplund, M. 2017, ApJ, 840, 99 Eggleton, P. P., Dearborn, D. S. P., \& Lattanzio, J. C. 2006, Sci, 314, 1580

Eisenstein, D. J., Weinberg, D. H., Agol, E., et al. 2011, AJ, 142, 72

El-Badry, K., Ting, Y.-S., Rix, H.-W., et al. 2018, MNRAS, 476, 528

Foy, R., \& Proust, D. 1981, A\&A, 99, 221

Gaia Collaboration, Brown, A. G. A., Vallenari, A., et al. 2018, A\&A, 616, A1

Gao, X., Lind, K., Amarsi, A. M., et al. 2018, MNRAS, 481, 2666

García Pérez, A. E., Allende Prieto, C., Holtzman, J. A., et al. 2016, AJ, 151,144

Geller, A. M., Latham, D. W., \& Mathieu, R. D. 2015, AJ, 150, 97

Gilmore, G., Randich, S., Asplund, M., et al. 2012, Msngr, 147, 25 González-Hernández, J. I., \& Bonifacio, P. 2009, A\&A, 497, 497

Gunn, J. E., Siegmund, W. A., Mannery, E. J., et al. 2006, AJ, 131, 2332

Hasselquist, S., Shetrone, M., Cunha, K., et al. 2016, ApJ, 833, 81

Holtzman, J. A., Hasselquist, S., Shetrone, M., et al. 2018, AJ, 156, 125

Holtzman, J. A., Shetrone, M., Johnson, J. A., et al. 2015, AJ, 150, 148 Iben, I., Jr. 1965, ApJ, 142, 1447

Jacobson, H. R., Pilachowski, C. A., \& Friel, E. D. 2011, AJ, 142, 59

Karakas, A. I., \& Lattanzio, J. C. 2014, PASA, 31, e030

King, J. R., Krishnamurthi, A., \& Pinsonneault, M. H. 2000, AJ, 119, 859

Korn, A. J., Grundahl, F., Richard, O., et al. 2007, ApJ, 671, 402

Lagarde, N., Decressin, T., Charbonnel, C., et al. 2012, A\&A, 543, A108

Lind, K., Korn, A. J., Barklem, P. S., \& Grundahl, F. 2008, A\&A, 490, 777

Liu, F., Asplund, M., Yong, D., et al. 2016, MNRAS, 463, 696

Majewski, S. R., Schiavon, R. P., Frinchaboy, P. M., et al. 2017, AJ, 154, 94

Martig, M., Fouesneau, M., Rix, H.-W., et al. 2016, MNRAS, 456, 3655

Masseron, T., \& Gilmore, G. 2015, MNRAS, 453, 1855

Masseron, T., Lagarde, N., Miglio, A., Elsworth, Y., \& Gilmore, G. 2017, MNRAS, 464, 3021

Mészáros, S., Allende Prieto, C., Edvardsson, B., et al. 2012, AJ, 144, 120

Mészáros, S., Holtzman, J., García Pérez, A. E., et al. 2013, AJ, 146, 133

Michaud, G. 1970, ApJ, 160, 641

Michaud, G. 1980, AJ, 85, 589

Michaud, G., Alecian, G., \& Richer, J. 2015, Atomic Diffusion in Stars (Cham: Springer International)

Michaud, G., Charland, Y., Vauclair, S., \& Vauclair, G. 1976, ApJ, 210, 447

Michaud, G., Richard, O., Richer, J., \& VandenBerg, D. A. 2004, ApJ, 606, 452

Ness, M., Hogg, D. W., Rix, H.-W., et al. 2016, ApJ, 823, 114 
Nidever, D. L., Holtzman, J. A., Allende Prieto, C., et al. 2015, AJ, 150, 173

Nordlander, T., Korn, A. J., Richard, O., \& Lind, K. 2012, ApJ, 753, 48

Önehag, A., Gustafsson, B., \& Korn, A. 2014, A\&A, 562, A102

Pancino, E., Carrera, R., Rossetti, E., \& Gallart, C. 2010, A\&A, 511, A56

Plez, B. 2012, Turbospectrum: Code for Spectral Synthesis, Astrophysics Source Code Library, ascl:1205.004

Price-Jones, N., \& Bovy, J. 2018, MNRAS, 475, 1410

Prša, A., Harmanec, P., Torres, G., et al. 2016, AJ, 152, 41

Randich, S., Gilmore, G. \& Gaia-ESO Consortium 2013, Msngr, 154, 47

Reddy, A. B. S., Giridhar, S., \& Lambert, D. L. 2012, MNRAS, 419, 1350

Richard, O., Michaud, G., \& Richer, J. 2002, ApJ, 580, 1100

Richard, O., Michaud, G., \& Richer, J. 2005, ApJ, 619, 538

Sarajedini, A., Mancone, C. L., Lauer, T. R., et al. 2009, AJ, 138, 184

Shetrone, M., Bizyaev, D., Lawler, J. E., et al. 2015, ApJS, 221, 24

Skrutskie, M. F., Cutri, R. M., Stiening, R., et al. 2006, AJ, 131, 1163

Smith, V. V., Cunha, K., Shetrone, M. D., et al. 2013, ApJ, 765, 16
Souto, D., Cunha, K., Smith, V., et al. 2016, ApJ, 830, 35

Souto, D., Cunha, K., Smith, V. V., et al. 2018, ApJ, 857, 14

Tautvaišiene, G., Edvardsson, B., Tuominen, I., \& Ilyin, I. 2000, A\&A, 360,499

Taylor, B. J. 2007, AJ, 134, 934

Vauclair, G., Vauclair, S., \& Michaud, G. 1978, ApJ, 223, 920

Vauclair, S., \& Vauclair, G. 1982, ARA\&A, 20, 37

Wilson, J. C., Hearty, F., Skrutskie, M. F., et al. 2010, Proc. SPIE, 7735, 77351C

Yadav, R. K. S., Bedin, L. R., Piotto, G., et al. 2008, A\&A, 484, 609

Yakut, K., Zima, W., Kalomeni, B., et al. 2009, A\&A, 503, 165

Zacharias, N., Finch, C., Subasavage, J., et al. 2015, AJ, 150, 101

Zamora, O., García-Hernández, D. A., Allende Prieto, C., et al. 2015, AJ, 149,181

Zasowski, G., Johnson, J. A., Frinchaboy, P. M., et al. 2013, AJ, 146, 81

Zhang, J., Shi, J., Pan, K., Allende Prieto, C., \& Liu, C. 2016, ApJ, 833, 137

Zhang, J., Shi, J., Pan, K., Allende Prieto, C., \& Liu, C. 2017, ApJ, 835, 90 